\title{
EXAMINING CEREBRAL HEMODYNAMICS IN A TWO-LAYER MODEL OF THE HUMAN HEAD USING BROADBAND NEAR-INFRARED SPECTROSCOPY
}

\author{
by \\ Olivia Pucci \\ B.MSc. (Hons.), University of Western Ontario, London, 2007
}

\author{
A thesis \\ presented to Ryerson University \\ in partial fulfillment of the \\ requirements for the degree of \\ Master of Science \\ in the Program of \\ Biomedical Physics
}

Toronto, Ontario, Canada, 2009

(C) Olivia Pucci 2009 


\section{Author's Declaration}

I hereby declare that I am the sole author of this thesis. I authorize Ryerson University to lend this thesis to other institutions of individuals for the purpose of scholarly research.

I further authorized Ryerson University to reproduce this thesis by photocopying or by other means, in total or in part, at the request of other institutions or individuals for the purpose of scholarly research. 


\title{
Examining Cerebral Hemodynamics in a Two-Layer Model of the Human Head using Broadband Near-Infrared Spectroscopy
}

\author{
Olivia Pucci \\ M.Sc., Biomedical Physics, Ryerson University, 2009
}

\section{Abstract}

The development of a continuous-wave method is presented, to quantify accurately the optical properties of a two-layer model of the human head using a broadband spectral approach. In particular, focus is put on the reconstruction of the absolute absorption and scattering properties of a two-layered phantom model of the human head with steady-state multi-distance measurements by performing differential fit analysis of the near-infrared (NIR) reflectance spectrum between $700 \mathrm{~nm}$ and $1000 \mathrm{~nm}$. The two-layer model approximation was fitted to experimental broadband absorbance measurements obtained from two-layered phantoms with known optical properties. Results demonstrated that the suggested method was able to determine the optical properties of the lower layer with minimal error at specific source-detector distances. Preliminary results on the non-invasive measurement of the optical properties of the adult human brain in a two-layer approximation are presented. Finally, a mobile wireless NIR device is used to measure changes in the temporal characteristics of cerebral hemodynamic responses to functional brain activity, in particular the effect of smoking. Results suggest that assuming homogeneous medium for the adult human head severely underestimates the changes in cerebral hemodynamics. Hence, it is important to take surrounding layers into consideration when performing cerebral measurements using NIR spectroscopy. 


\section{Acknowledgements}

Many thanks to my supervisor, Dr. Toronov, who taught me so much and allowed me to grow as a scientist and as a student. You never gave up on me, and always demanded the best from me, and for that I am thankful. To my supervisory committee, thank you for taking time to give me support, suggestions and your patients during those long meetings.

I would like to express my deepest appreciation and love to my parents whom without their support I would not be where I am today. Their love and encouragement through the toughest times got me through those meltdown moments. I always hope to make them proud of me. To my brother, who was my guinea pig for some of my experiments and my chauffer, thank you for your help and your forehead.

To my grandparents who never hesitated to help in any way they could. For always encouraging me and praying for me. I love you very much. I would like to thank the entire Sobczyk and Staton family, who always had an encouraging attitude and never hesitated to lend a listening ear. Thank you for all your support and for all the food and shelter you provided me. As well as those amazing relaxing Florida trips, and surprise presents. To my friends, Jenny and Amy who always wanted to know my progress and always had optimistic outlook on everything. Thank you for all the fond memories over the years, I will always remember and cherish them.

To my fellow classmates, with the fun we had trying to figure out that one problem to an assignment due in three hours, to the encouragement and healthy competition we gave each other. It was truly an experience.

Finally and most importantly, to my Matthew, who put up with my frustration and always encouraged me to never give up and helped me to relax and breathe. Our time together during this journey was fun and I will always love you. You are the most patient and understanding human being I know, and without you I do not think I would have gotten through it and thank you for reminding me that all work and no breaks will make Olivia a very unhappy girl. I am excited for the new journeys ahead of us. 


\section{Table of Contents}

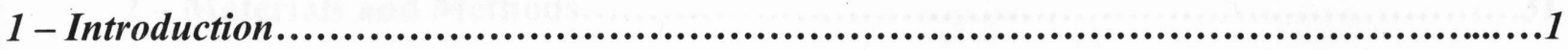

1.1 - Light Transport in Tissue.......................................................

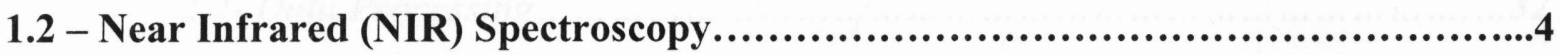

1.2.1-Different NIR Spectroscopy Measuring Techniques ......................................5

1.2.1.1-Time-Resolved Spectroscopy ................................................5

1.2.1.2-Frequency Domain Spectroscopy ........................................6

1.2.1.3-Continuous Wave Spectroscopy ............................................

1.2.2-NIR Spectroscopy of Chromophores ................................................. 8

1.2.2.1-Oxyhemoglobin and Deoxyhemoglobin Absorption Spectra ..................9

1.2.2.2-Modified Beer Lambert Law ..................................................11

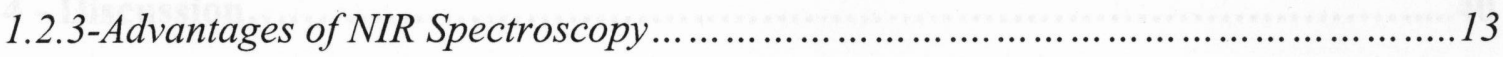

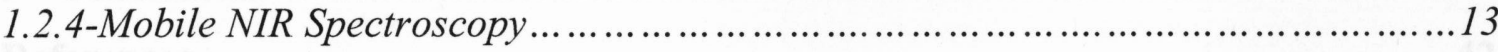

1.3 - NIR Spectroscopy in Medicine...................................................13

1.4 - NIR Spectroscopy and Cerebral Hemodynamics..................................14

1.5 - Current Alternate Techniques Used for Cerebral Imaging.........................16

1.5.1 - Electro- and Magneto- encephalography ...........................................16

1.5.2 - Positron Emission Tomography ..................................................... 17

1.5.3 - Function Magnetic Resonance Imaging ........................................... 18

1.5.4 - Diffuse Optical Tomography ....................................................... 19

1.6 - Anatomy and Physiology of the Human Head......................................20

1.7 -NIR Spectroscopy and Current Two-Layered Models of the Human Head...........21

1.8 - Naturally Administered Smoke and Cerebral Hemodynamics......................23

1.9 - Thesis Organization and Objectives..............................................25

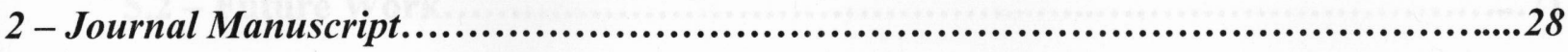




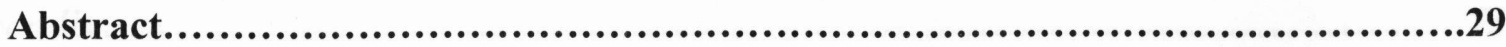

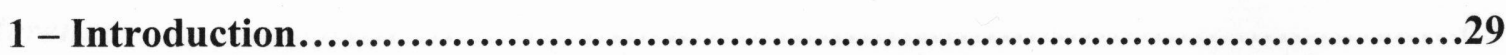

2 - Materials and Methods...........................................................31

2.1-Broadband Optical Spectroscopy of Tissue: The Light Source and Detector31

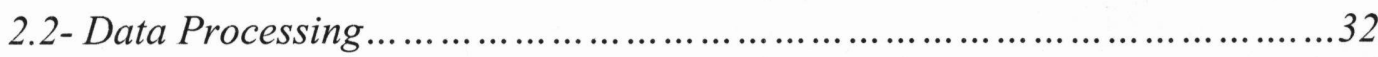

2.2.1- Broadband Approach to Forward Modeling............................32

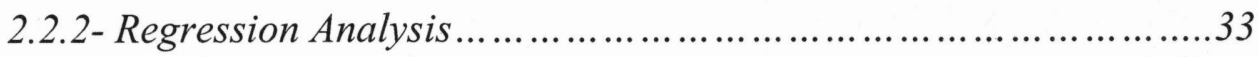

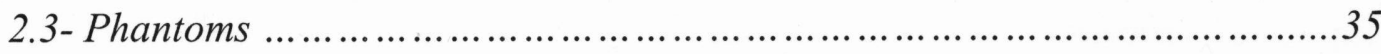

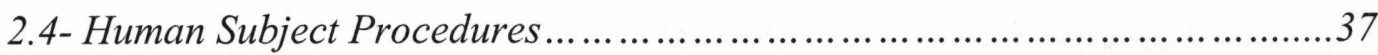

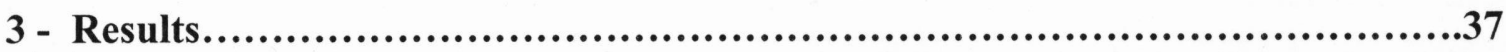

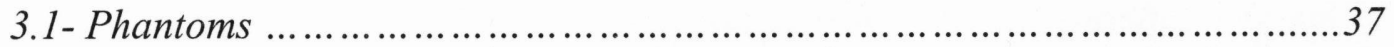

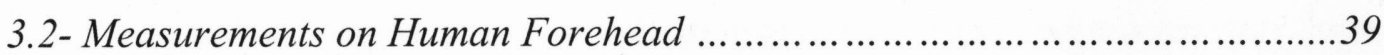

4 - Discussion.....................................................................40

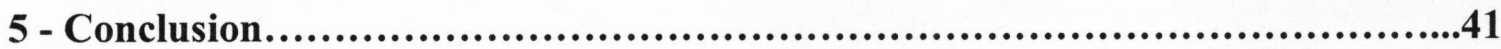

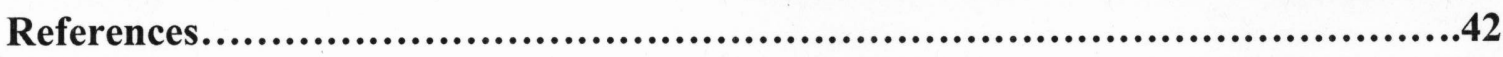

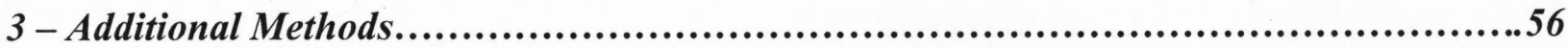

3.1 - Differential Path Length Calculation..............................................56

3.2 - Smoking Measurements.......................................................58

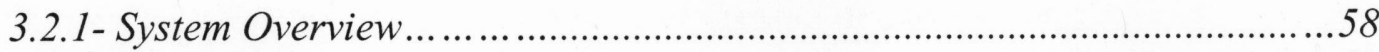

3.2.2- Human Subjects and Experimental Protocol.........................................59

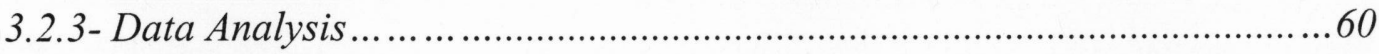

4 - Differential Path Length Factor and Mobile Sensor Results and Discussion..............62

4.1 - Differential Path Length Factor and Mobile Sensor Results......................62

4.2 - Differential Path Length Factor and Mobile Sensor Discussion...................66

5 - Conclusions and Future Work......................................................68

5.1 - Conclusions....................................................................68

5.2 - Future Work ..................................................................... 


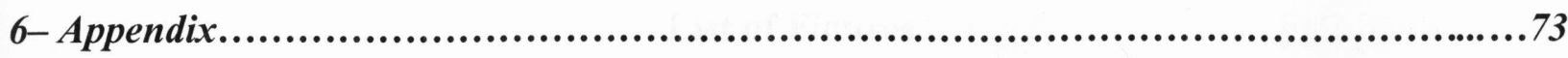

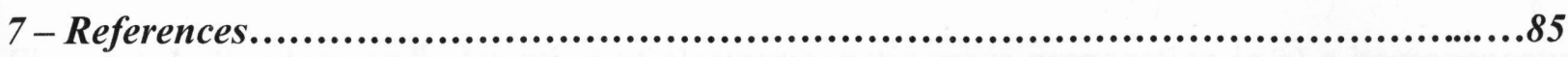




\section{List of Figures}

Figure 1-1- Random walk depiction of electromagnetic wave propagation in a) a homogeneous medium [6] b) in biological tissue [4] .................................................

Figure 1-2- Representation of how light travels from source to detector following a banana

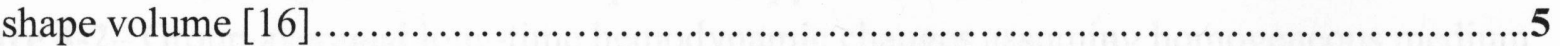

Figure 1-3- Depiction of the influences of scattering within a medium for Time-Resolved

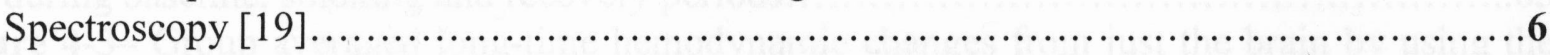

Figure 1-4- Phase shift and waveform parameters used in Frequency Domain Spectroscopy. Found at Rolfe, P. [19] ............................................................

Figure 1-5- a) Molecular composition of hemoglobin found at Mader, S.S [25] b) molecular structure of a heme molecule found at Brown et. al. [23] .................................9

Figure 1-6- Specific absorption coefficient spectra of deoxyhemoglobin, oxyhemoglobin, lipid and water according to wavelength in the near-infrared optical window [26-29] ...........10

Figure 1-7- How light travels from source to detector when placed on top of the human head

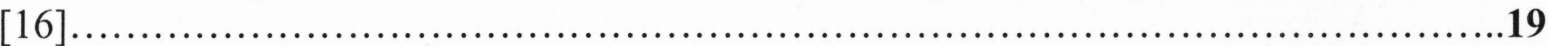

Figure 1-8- a) The anatomy of the human head which demonstrates the many layers which surround the brain. b) The brain itself is composed of white and gray matter. Found at Schünke et. al [59] ..................................................................

Figure 1-9- Two-layer medium approximate model of the human head, where the top layer represents the scull and the surrounding tissue layers as one homogeneous effective layer,

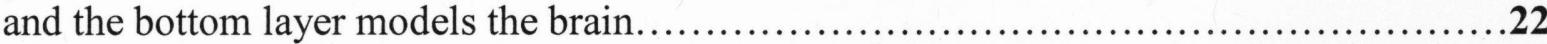

Figure 1-10- Schematic diagram of methodology organization. Approach is based on three main experiments: 1) Phantom validation 2) In vivo baseline measurements and 3) Changes in

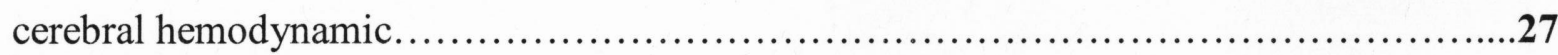

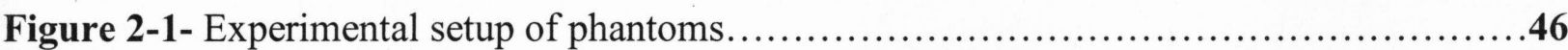

Figure 2-2- Relative absorbance spectra of phantoms to demonstrate sensitivity of system to bottom layer in a two-layered model.

Figure 2-3- Absorption spectra of each homogeneous phantom...........................48

Figure 2-4- Reduced Scattering Coefficient Spectra of homogeneous phantoms.

Figure 2-5- Silicone absorption spectrum and Molar extinction of carbon black...............50

Figure 2-6- Theoretical differential absorbance sensitivity to relative changes in $\mu_{\mathrm{a}}$ of carbon black with constant scattering for two-layer phantom model.............................51

Figure 2-7- The experimental spectral derivative of the absorbance for the Two-Layer I phantom and the theoretical fit.

Figure 2-8- A comparison of the reconstructed absorption spectrum of the Type II bottom layer of the Two-Layer I phantom with the absorption spectrum obtained from measurements on the homogeneous Type II medium.

Figure 2-9- A comparison of the reconstructed absorption spectrum of the Type I bottom layer of the Two-Layer II phantom with the absorption spectrum obtained from measurements on the homogeneous Type I medium.

Figure 2-10- The experimental spectral derivative of the absorbance of human forehead at SD distance of $25 \mathrm{~mm}$ and the theoretical fit using the homogeneous model. 
Figure 3-1- a) Mobile NIR sensor with portable phone b-d) Mobile sensor light emitters and detectors.

Figure 4-1- Differential path length factor for the second layer as a function of wavelength calculated using baseline optical properties of the bottom layer of a two-layered model of the human head, using broadband NIR spectroscopy at SD distance of $25 \mathrm{~mm}$. .62

Figure 4-2- Group averaged long-time hemodynamic changes assuming homogeneous medium during baseline, smoking and recovery periods. .65

Figure 4-3- Group averaged long-time hemodynamic changes from just the brain by using the DPF's at $730 \mathrm{~nm}$ and $830 \mathrm{~nm}$ from Figure 4-1, during baseline, smoking and recovery periods. .65 


\section{List of Tables}

Table 2-1- Recovered optical properties for homogeneous phantoms with the higher optical properties (Type I) and the phantoms with lower optical properties (Type II), and two-layered model fitting for bottom layer having lower optical properties than top layer (Two-Layer I) and vise versa for Two-Layered II. Reference intensity was measured at the smaller source/detector distance and sample intensity at the larger distance....................................... 


\section{1 - Introduction}

\section{1 - Light Transport in Tissue}

Light is a set of electromagnetic waves, and biological tissue is a highly scattering heterogeneous medium composed of many cells, organelles, and membranes that all have different indices of refractions [1]. Therefore, when light enters tissue, the electromagnetic waves will refract at different angles when encountering a random interface [1]. When an electromagnetic wave in the form of a beam enters biological tissue, it splits into infinitely many new beams due to the scattering (Figure 1-1). At these conditions the properties characterizing the medium are the scattering and absorption [2]. Some of the light energy is absorbed and converted into heat and chemical energy. Tissues absorption is represented by the absorption coefficient $\mu_{a}$, which is defined as the linear attenuation rate of irradiance per unit of length [3]. The scattering characteristic of tissue is described using the scattering coefficient $\mu_{s}$ which represents the probability per unit path length that a light wave will encounter a scatterer [3]. The inverse of $\mu_{s}$ has units of length and can be interpreted as the mean free path of a wave. The angle at which the wave scatters is a function of the size and shape of the scatterer as well as the wavelength of the electromagnetic wave, in addition to the angle at which the incident wave reaches the particle interface surface [4]. This introduces a new parameter to describe the fate of electromagnetic waves when interacting with tissue, which is called the anisotropy factor of scattering, or the mean cosine of the scattering angle, $g$. Different tissues interact with light according to their specific absorption and scattering properties as well as the tissue's anisotropy factor [5]. In order to obtain any information on tissue from an optical measurement, a model of light transport in tissue is required. 


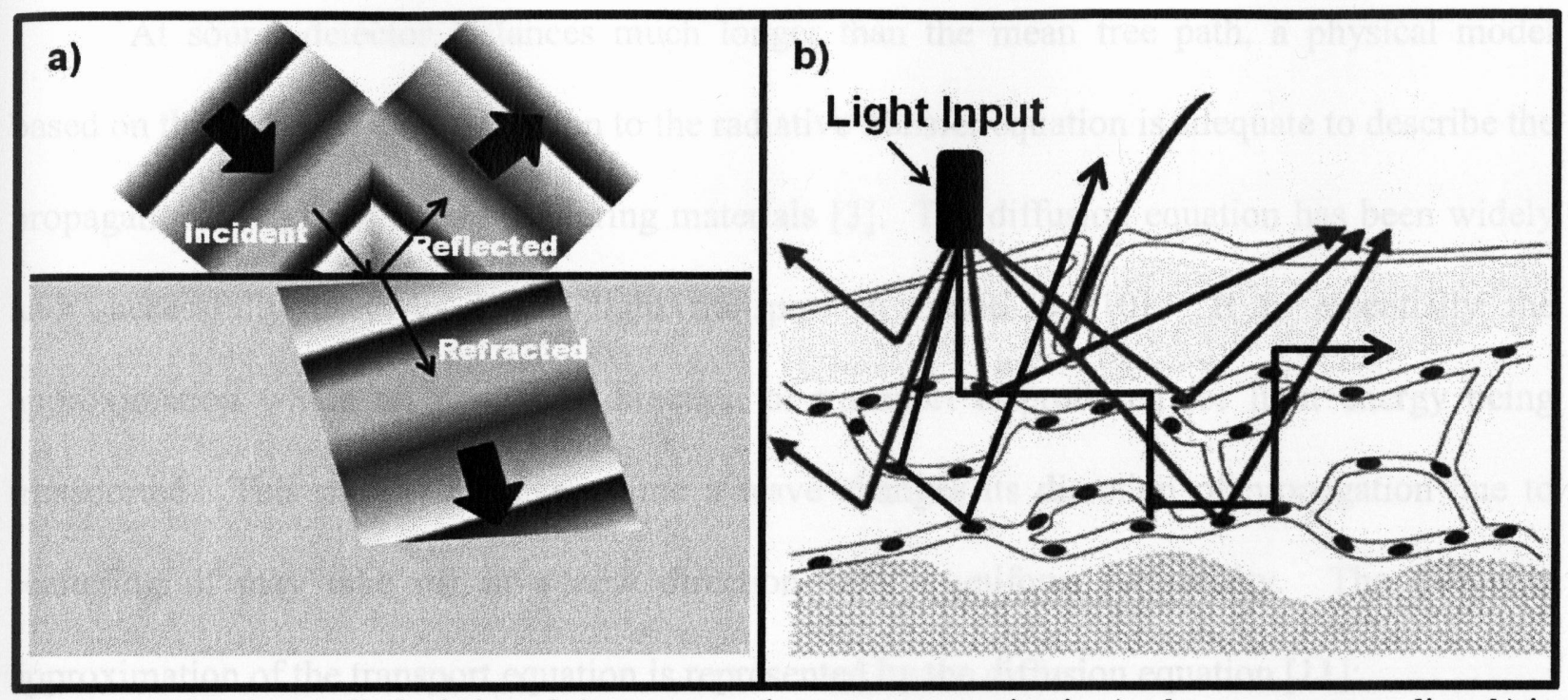

Figure 1-1- Random walk depiction of electromagnetic wave propagation in a) a homogeneous medium b) in biological tissue. When electromagnetic waves in the form of a beam enter biological tissue, it splits into infinitely many new beams due to the scattering. When electromagnetic waves are scattered it changes the direction of propagation of the wave. a) From Goode, T.L et. al. [6] and b) From Hecht, E. [4].

The fundamental equation governing light transport in highly scattering media, is the radiative transfer equation (RTE) which takes account of scattering for inhomogeneities and of absorption by the medium $[7,8]$. The RTE has been used successfully to model light transport in diffusive media [3]:

$$
\left\{\widehat{s} \cdot \nabla+\mu_{a}(r)+\mu_{s}(r)+\frac{\partial}{c \partial t}\right\} I(r, \widehat{s}, t)=q(r, \widehat{s}, t)+\mu_{s}(r) \int\left(\left(f\left(\widehat{s}^{\prime}, \widehat{s}, t\right) \cdot I(r, \widehat{s}, t)\right)\right) d^{2} \widehat{s}^{\prime} .
$$

The RTE is a conservation equation that describes the change of the radiance $I(r, \hat{s}, t)$ at time $t$ at position $r$ into direction $\hat{s} . q(r, \hat{s}, t)$ is the source term at $\mathrm{r}$ at time $\mathrm{t}$ travelling in direction $\hat{s}, c$ is the speed of light in tissue, and $f(\hat{s}, \hat{s}, t)$ is the scattering phase function which gives the probability of a wave scattering from direction $\hat{s}$ ' to direction $\hat{s}$. 
At source-detector distances much longer than the mean free path, a physical model based on the diffusion approximation to the radiative transfer equation is adequate to describe the propagation of light in highly scattering materials [3]. The diffusion equation has been widely and successfully used to model light transport in tissue $[9,10]$. It is essentially the approximation where no particular direction of transport is preferred for light energy being transported. This means that every time a wave changes its direction of propagation due to scattering, it may take off in a new direction with a uniform probability. The diffusion approximation of the transport equation is represented by the diffusion equation [11]:

$$
c \mu_{a} U(r, t)+\frac{\partial U(r, t)}{\partial t}-c D \Delta U(r, t)=Q(r, t)
$$

where $U(r, t)$ represents the "photon density", i.e. the volume energy of light, in units of $\mathrm{J} / \mathrm{cm}^{3}$, $\mathrm{D}=\left(3 \mu_{a}+3 \mu_{s}^{\prime}\right)^{-1}$ is the diffusion coefficient in units of $\mathrm{cm}^{2} / \mathrm{s}$ and $\mu_{s}^{\prime}$ represents the reduced scattering coefficient $\mu_{s}{ }^{\prime}=\mu_{s}(1-g)$. The purpose of $\mu_{s}{ }^{\prime}$, is to describe the diffusion of electromagnetic waves in a random walk of step size of $1 / \mu_{s}{ }^{\prime}[\mathrm{cm}]$ where each step involves isotropic scattering [1]. The source term $Q(r, t)$ in general represents an intensity modulated source. The diffusion approximation can be applied to any medium where the scattering is dominant over the absorption, i.e. $\mu_{s}{ }^{\prime} » \mu_{a}$ [3]. This condition is generally true for tissues (such as muscle and skin) where near-infrared (NIR) wavelength between 600 to $1000 \mathrm{~nm}$ is used. Hence the diffusion approximation becomes an appropriate approximation for many biomedical applications at NIR wavelengths [3]. Examples of tissue for which this approximation does not hold true include cerebrospinal fluid, eye lenses and nails. 


\section{2 - Near Infrared Spectroscopy}

Near-infrared spectroscopy (NIRS) along with other neuroimaging methods has inspired the development of biophysical models and hypotheses to gain a better understanding of brain physiology [12]. These methods have shown promising information on the hemodynamic processes of the brain. In the NIR region of the electromagnetic spectrum, between $700-900 \mathrm{~nm}$, a significant amount of light can be transmitted through biological tissue over longer distances [13]. The combined attributes of low absorption and high scattering in tissue allows for NIR light of penetrate deep within tissue noninvasively, on the order of centimetres [13]. A typical tissue spectrometer has a light source that emits light of different NIR wavelengths on the scalp, and detectors that detect backscattered light [14] as depicted in Figure 1-2. When an NIR light penetrates a medium, the light scatters in all directions. However, when a detector is placed at a certain distance from the source, most photons travel, from the source to the detector in a random path following a banana shaped volume (Figure 1-2). This is due to the fact that scattering increases the optical path length $[15,16]$. Changes in the amount of backscattered light can be transformed into the concentration changes in oxyhemoglobin $(\mathrm{HbO} 2)$ and deoxyhemoglobin (HHb) using a modified Beer-Lambert Law [15, 17]. 


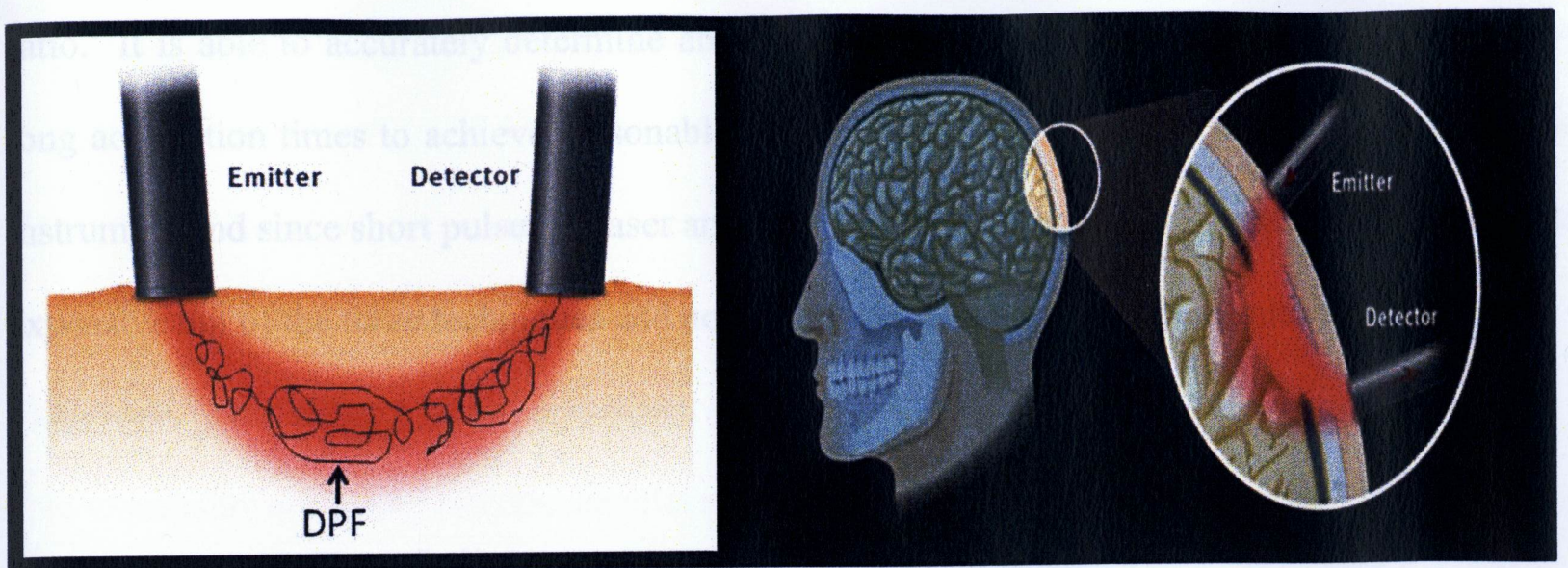

Figure 1-2- Representation of how light travels from source to detector following a banana shape volume. To account for the average increase in pathlength of the very complex beam trajectory within the banana shape volume, a factor is introduced known as the differential path length factor (DPF). From Izzetoglu et. al. [16].

\subsubsection{Different NIR Spectroscopy Measuring Techniques}

Progress in NIRS and in the imaging of tissues has demonstrated that the optical properties in a turbid medium can be obtained using NIR [18]. There are three main different approaches to implementing NIRS: Time-Resolved Spectroscopy (TRS), Frequency Domain Spectroscopy (FDS) and Continuous Wave Spectroscopy (CWS). Each has advantages and disadvantages when used for different applications.

\subsubsection{1 - Time-Resolved Spectroscopy}

Time-Resolved Spectroscopy transmits short light pulses into a medium and measures the light after it passes through the medium. The group of light paths produced by scattering leads to a broadening of the pulse. The time at which the maximum detected intensity occurs relative to the input pulse is the mean arrival time of light waves and is used to calculate mean optical path length [19]. The system detects the temporal distribution of light energy as light waves arrive to the detector; the shape of this distribution provides information about tissue scattering and absorption (Figure 1-3) [10]. TRS has decent spatial resolution but a low signal-to-noise (SNR) 
ratio. It is able to accurately determine absorption and scattering properties, however requires long acquisition times to achieve reasonable SNR ratios. TRS also requires stabilization of the instrument and since short pulses of laser and photon counting detection is required it is the most expensive out of the three techniques and not directly suitable for clinical monitoring $[10,19]$.

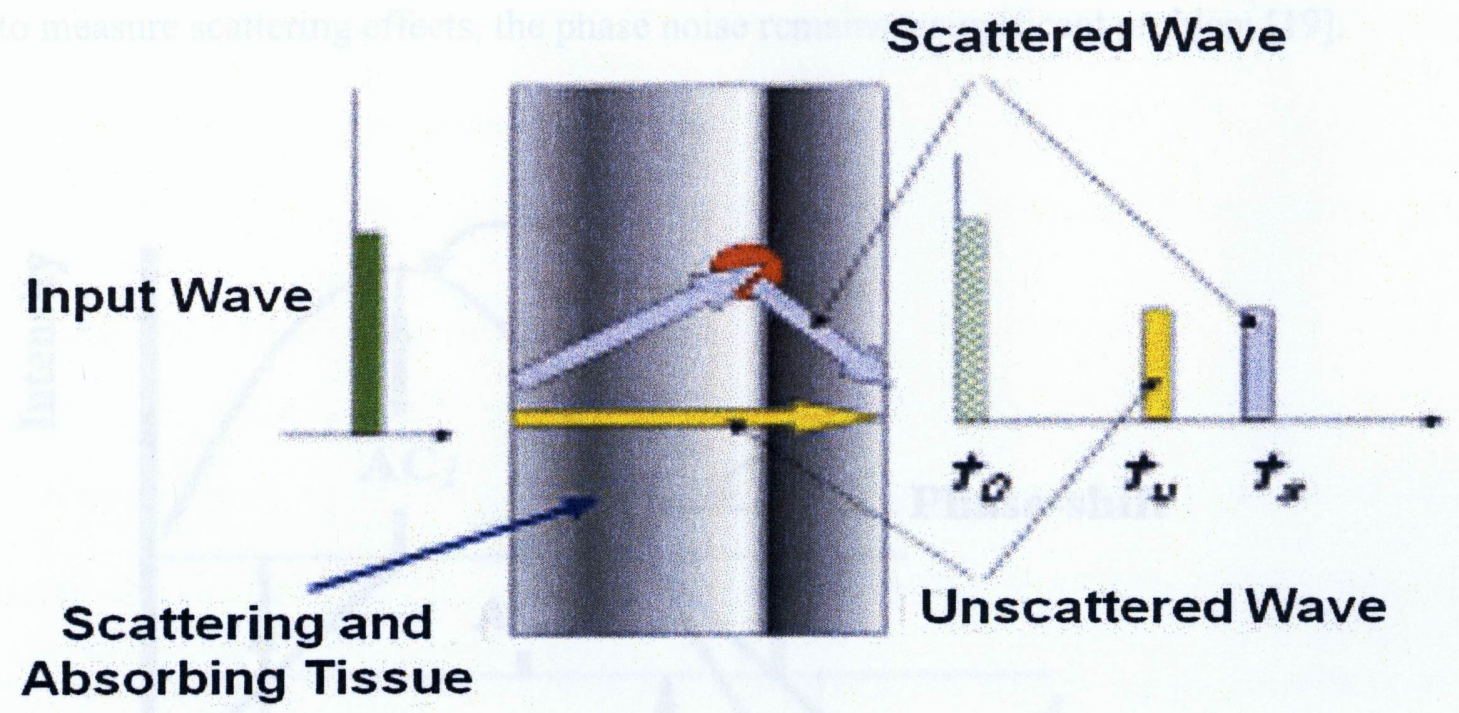

Figure 1-3- Depiction of the influences of scattering within a medium for TRS. A short pulse of light will be detected at a time dependent upon scattering events, from Rolfe, P. [19].

\subsubsection{2 - Frequency Domain Spectroscopy}

Frequency Domain Spectroscopy transmits light from a sinusoidally modulated source into the tissue and the detected energy exhibits a phase shift in comparison with the modulating signal, due to the propagation delay as well as attenuation due to absorption and scattering [19]. The phase shift allows for the optical path length to be calculated as shown in Figure 1-4. Measurements of the absorption and scattering coefficients of the homogenous infinite medium can be calculated using the diffusion approximation expressions for the phase shift, and the DC 
and $\mathrm{AC}$ components of the photon density [19]. Measurements at a single modulation frequency can be sufficient to isolate the contribution of the scattering from that of the absorption. However, this is strictly true only for homogeneous medium and measurement locations of 7-8 $\mathrm{mm}$ or farther from the light source [19]. FDS provides the same information as TRS, however it offers a faster acquisition time and cheaper hardware than TRS. While FDS is able to measure scattering effects, the phase noise remains a significant problem [19].

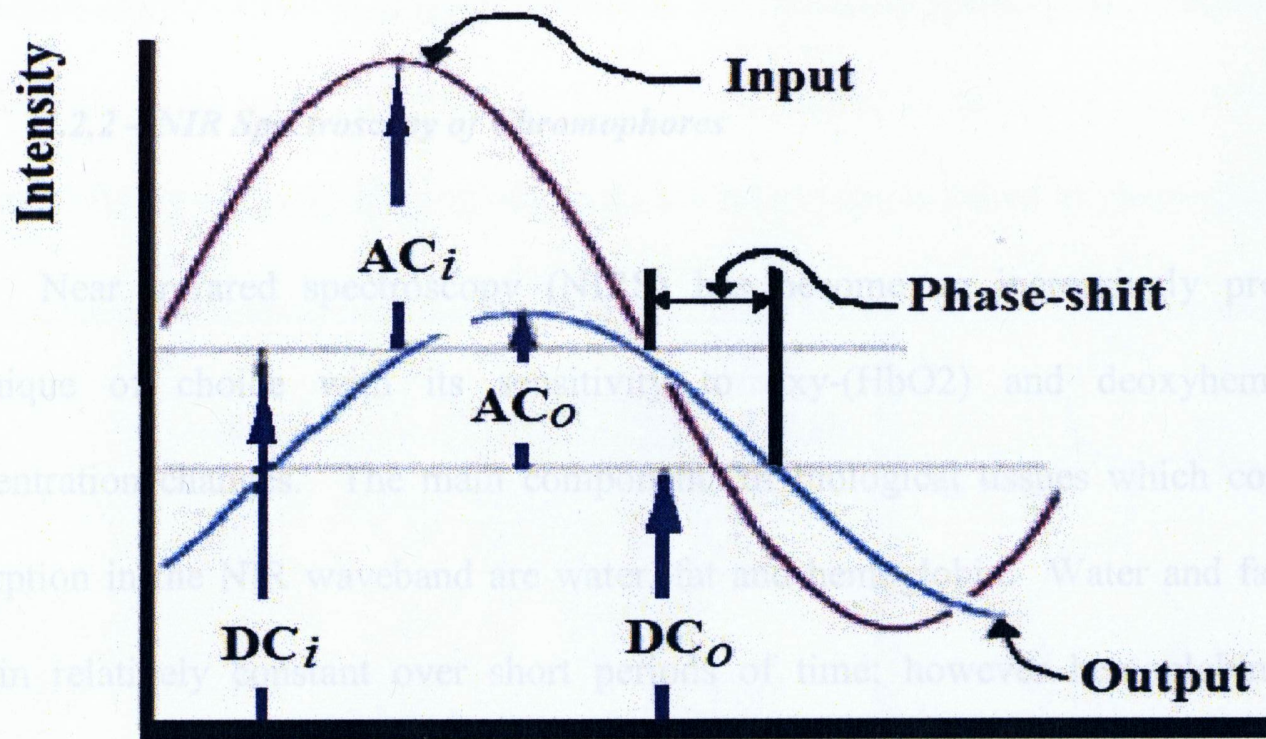

\section{Time}

Figure 1-4- Phase shift and waveform parameters used in FDS. Found at Rolfe, P. [19].

\subsubsection{3 - Continuos Wave Spectroscopy}

Continuous Wave Spectroscopy (CWS) consists of a CW light source, which transmits light waves at constant amplitude, and a detector that that measures the fluence of the attenuated light after it passes through a medium [15]. CW systems measure only the amplitude decay of 
the incident light and do not provide sufficient information to quantify baseline absorption and scattering separately at an arbitrary wavelength [19]. CWS is the least expensive out of the three techniques mentioned; however it is difficult to distinguish contributions of absorption and scattering to the light attenuation [15]. CW imaging can achieve image frame rates faster than $100 \mathrm{~Hz}$ at practical SNR. CW measurements such as those first used by Jobsis [2] and Chance [20], as well as others have shown its ability to quantitatively monitor the absorption optical properties if the scattering properties are known [20].

\subsection{2 - NIR Spectroscopy of Chromophores}

Near infrared spectroscopy (NIRS) has become an increasingly preferred imaging technique of choice with its sensitivity to oxy-(HbO2) and deoxyhemoglobin ( $\mathrm{HHb})$ concentration changes. The main components of biological tissues which contribute to light absorption in the NIR waveband are water, fat and hemoglobin. Water and fat concentrations remain relatively constant over short periods of time; however hemoglobin changes occur relative to the function and metabolism of the tissue [2]. Hence corresponding changes in absorption by hemoglobin can provide clinically useful information about different physiological parameters such as oxygenation levels and blood flow [21].

Visible and NIR molecular absorption spectra result from the electronic transitions broadened by vibrational motions of the atomic nuclei [22]. The chromophore absorption coefficient at a particular wavelength is determined by the product of the specific absorption coefficient (also known as the extinction coefficient) of the chromophore and the concentration 
of chromophore molecules [22]. The total tissue absorption coefficient is also equal to the sum of absorption coefficients of all chromophores.

\subsubsection{1 - Oxyhemoglobin and Deoxyhemoglobin Absorption Spectra}

Hemoglobin is a molecule in a red blood cell with the role of delivering oxygen to tissue cells [23]. Hemoglobin is composed of four heme molecules and a protein group known as a globin (Figure 1-3). The heme molecule in hemoglobin is responsible for binding oxygen molecules and has its own characteristic NIR absorption spectrum [24]. The presence of oxygen significantly alters these properties. A hemoglobin molecule can carry four molecules of oxygen as seen in figure 1-5 [24]. Hemoglobin with bound oxygen is called oxyhemoglobin.

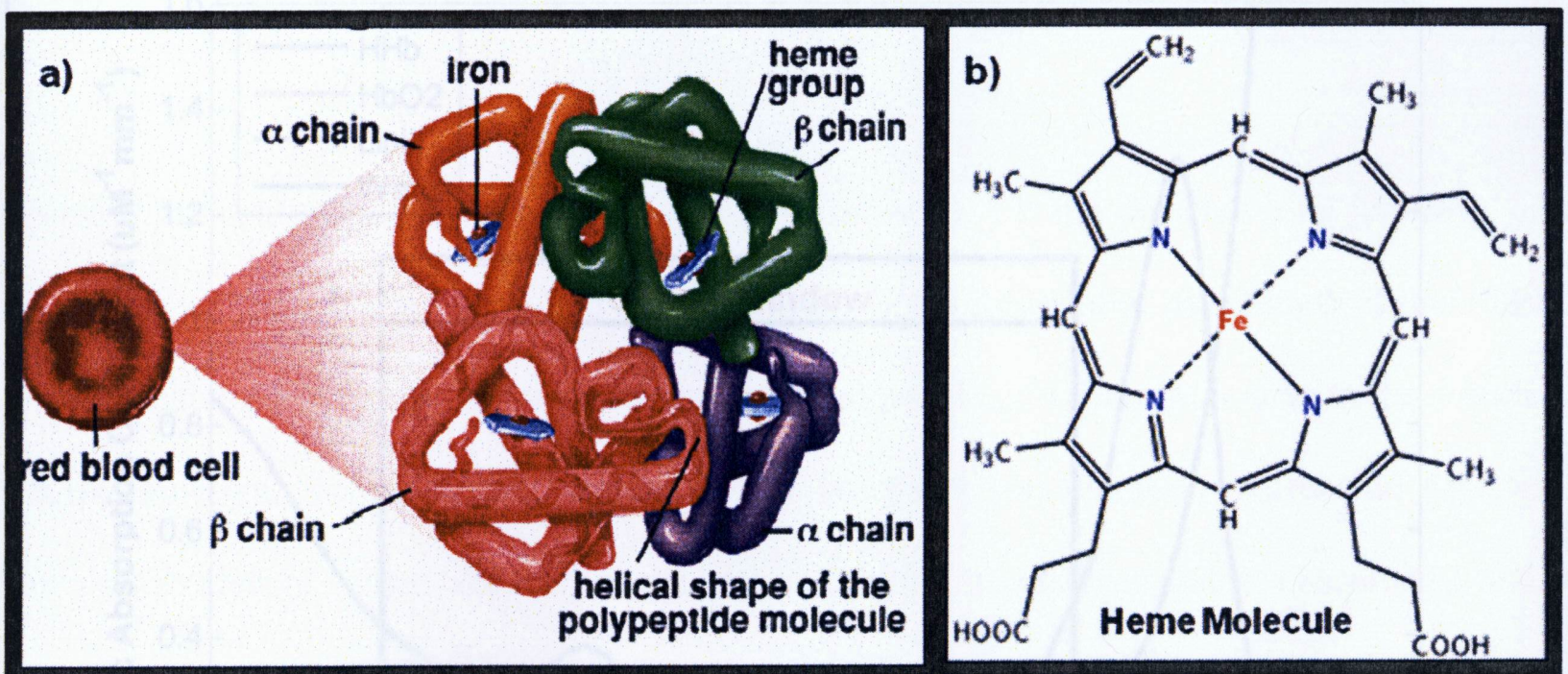

Figure 1-5- a) Molecular composition of hemoglobin found at Mader, S.S [25] b) molecular structure of a heme molecule found at Brown et. al. [23].

In the NIR spectra, at specific wavelengths in the window of 700 to $900 \mathrm{~nm}$ the absorption of deoxyhemoglobin $(\mathrm{HHb})$, oxyhemoglobin $(\mathrm{HbO} 2)$, lipid and water is relatively low (optical window displayed in Figure 1-6) and therefore the light at these wavelengths can penetrate 
deeper or thicker tissues. On the other hand, using NIR extinction coefficients of these chromophores known from the literature [26-29], the absolute concentrations or changes in the concentrations of species can be measured. Water and lipid absorbs NIR light at higher wavelengths, around $960 \mathrm{~nm}$ and $940 \mathrm{~nm}$ respectively [28, 29]. $\mathrm{HbO} 2$ has a specific NIR absorption spectrum with a maximum at approximately $870 \mathrm{~nm}$ (see Figure 1-6). Once oxygen is delivered to the specific tissue, the hemoglobin no longer has bound oxygen to its heme groups and hence is known as $\mathrm{HHb}$ which has specific absorption maximum near 760nm [18]. Benefits arise from these characteristic spectra of $\mathrm{HbO} 2$ and $\mathrm{HHb}$ (Figure 1-6), which is the ability to measure oxygenation saturation of blood noninvasively using optical means [30].

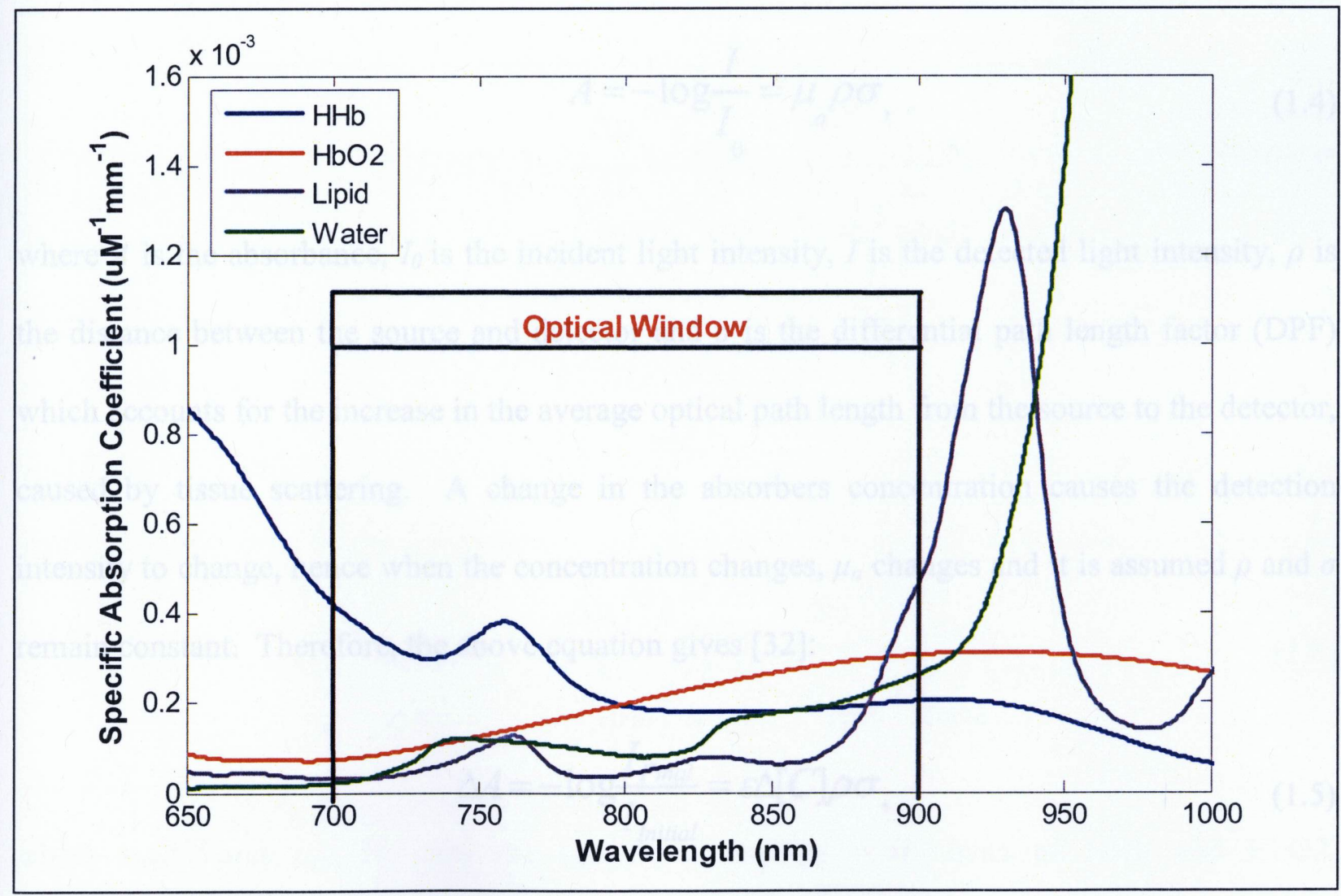

Figure 1-6- Specific absorption coefficient spectra of $\mathrm{HHb}, \mathrm{HbO2}$, lipid and water according to wavelength in the NIR optical window [26-29]. 
For spectroscopy purposes, experimenters have used Beer-Lambert's law to develop a relationship between light absorption as a function of hemoglobin concentration. This theory applies to any kind of absorption in a scattering medium [31]. Beer-Lambert's Law states that:

$$
\mu_{a}=\varepsilon C
$$

Where $\mu_{a}$ is the absorption coefficient $\left(\mathrm{mm}^{-1}\right), C$ is the concentration $(\mu \mathrm{M})$ and $\varepsilon$ is the specific absorption coefficient of the absorber per unit of path length and concentration $\left(\mu \mathrm{M}^{-1} \mathrm{~mm}^{-1}\right)$. Chromophore concentration can be quantified using a modified Beer-Lambert law [18, 31, 32]:

$$
A=-\log \frac{I}{I_{0}}=\mu_{a} \rho \sigma,
$$

where $A$ is the absorbance, $I_{0}$ is the incident light intensity, $I$ is the detected light intensity, $\rho$ is the distance between the source and detector and $\sigma$ is the differential path length factor (DPF) which accounts for the increase in the average optical path length from the source to the detector, caused by tissue scattering. A change in the absorbers concentration causes the detection intensity to change, hence when the concentration changes, $\mu_{a}$ changes and it is assumed $\rho$ and $\sigma$ remain constant. Therefore, the above equation gives [32]:

$$
\Delta A=-\log \frac{I_{\text {Final }}}{I_{\text {Initial }}}=\varepsilon \Delta[C] \rho \sigma,
$$

where $\Delta A=A_{\text {Final }}-A_{\text {Initial }}$ is the change in the absorbance, $I_{\text {Final }}$ and $I_{\text {Initial }}$ are the measured intensities before and after the concentration change, and $\Delta[C]$ is the change in concentration. 
To apply this theory to changes in $\mathrm{HbO} 2$ and $\mathrm{HHb}$ concentrations, equation (1.5) must be expressed as:

$$
\Delta A=\Delta \mu_{a_{\lambda}} \rho \sigma_{\lambda}=\left(\varepsilon_{\lambda}^{H b O 2} \Delta[H b O 2]+\varepsilon_{\lambda}^{H H b} \Delta[H H b]\right) \rho \sigma_{\lambda},
$$

where $\lambda$ represents a particular wavelength. Equation (1.6) accounts for independent concentration changes in $\mathrm{HbO} 2$ and $\mathrm{HHb}$ at those specific wavelengths and is dependent on the baseline optical properties. Once the temporal changes in the absorption coefficients $\Delta \mu_{a}^{\lambda 1,2}$ of the tissue at the wavelengths $\lambda_{1}$ and $\lambda_{2}$ are computed, the changes in the hemoglobin content can be obtained from (1.6) in the form [11, 32]:

$$
\Delta\left[\mathrm{HbO}_{2}\right]=\frac{\Delta \mu_{\mathrm{a}}^{\lambda 1} \varepsilon_{\mathrm{HHb}}^{\lambda 2}-\Delta \mu_{\mathrm{a}}^{\lambda 2} \varepsilon_{\mathrm{HHb}}^{\lambda 1}}{\varepsilon_{\mathrm{HbO} 2}^{\lambda 1} \varepsilon_{\mathrm{HHb}}^{\lambda 2}-\varepsilon_{\mathrm{HHb}}^{\lambda 1} \varepsilon_{\mathrm{HbO} 2}^{\lambda 2}}
$$

and,

$$
\Delta[\mathrm{HHb}]=\frac{\Delta \mu_{\mathrm{a}}^{\lambda 1} \varepsilon_{\mathrm{HbO} 2}^{\lambda 2}-\Delta \mu_{\mathrm{a}}^{\lambda 2} \varepsilon_{\mathrm{HbO} 2}^{\lambda 1}}{\varepsilon_{\mathrm{HHb}}^{\lambda 1} \varepsilon_{\mathrm{HbO} 2}^{\lambda 2}-\varepsilon_{\mathrm{HHb}}^{\lambda 1} \varepsilon_{\mathrm{HbO} 2}^{\lambda 2}}
$$

where $\varepsilon_{\mathrm{HHb}}^{\lambda l, 2}$ and $\varepsilon_{02 \mathrm{Hb}}^{\lambda l, 2}$ are the specific absorption coefficients of $\mathrm{HHb}$ and $\mathrm{HbO}$, respectively, at wavelengths $\lambda_{I}$ and $\lambda_{2}$. 


\subsubsection{Advantages of NIR Spectroscopy}

There are several advantages of NIRS over other imaging modalities. NIRS provides accurate quantitative information about physiological parameters, such as the hemodynamic parameters, that are not available in other modalities and has a high temporal resolution, on the order of milliseconds. NIR is sensitive to features such as scattering and changes in the refractive index which results in the sensitivity to neuronal processes $[18,31,33]$.

\subsection{4-Mobile NIR Spectroscopy}

Most of NIRS systems use electrical or optical cables to connect the sensor attached to the body of the subject to the acquisition device. These cables cause motion artifacts and can be inconvenient in some applications, for example in situations where NIRS is used to monitor freely moving subjects such as those exercising (to monitor muscle oxygenation), and subjects in social interaction (to monitor brain activity) $[15,34]$. To overcome these limitations mobile NIRS systems were created recently, allowing for advancements in experimental data being acquired from test subjects in their natural environment [34]. Having the ability to monitor physiological changes while subjects perform daily routines allows for parameters to be measured with much more comfort and accuracy than with fibers, and opens new fields of application such as in medical emergency situations.

\section{3 - NIR Spectroscopy in Medicine}

Over the last three decades, many researchers evaluated NIRS in medical applications. The most promising attribute of NIRS is the ability to measure fast changes in optical properties of cerebral tissue [35]. NIRS is being promoted as an effective method of monitoring cerebral 
oxygenation during carotid, cerebrovascular and cardiopulmonary bypass surgery in head-injured and other intensive care patients [36]. In addition to the investigation of functional cerebral hemodynamics in humans, several studies have shown NIRS being tested in clinical applications. One study by Steinhoff et al. used a NIR device to evaluate patients with epilepsy [37]. They were able to conclude that NIRS is a simple, cost-effective, non-invasive addition to determining primary epileptogenic zones in temporal lobe epilepsy. Kirkpatrick et al. [38] discovered promising features to his use of NIRS in adult subjects that had head injuries and carbon dioxide stress testing [38].

Since recently, NIRS is being used in pediatric critical care, to help deal with cardiac surgery post-op [34]. Indeed, NIRS is able to measure venous oxygen saturation (SVO2), which is determined by the cardiac output, as well as other parameters (for example hemoglobin and oxygen uptake). Therefore, following NIRS gives critical care physicians a notion of the cardiac output [39]. NIRS can also be used to quantify blood volume and oxygenation, a form of oximetry. Different applications of NIR oximetry include detection of illnesses which affect the blood circulation (e.g. peripheral vascular disease), the detection and assessment of breast tumors, and the optimization of training in sports medicine [35]. NIRS has advantages over other techniques because it is non-invasive, painless and doesn't rely on ionizing radiation.

\subsection{NIR Spectroscopy and Cerebral Hemodynamics}

The brain is the most dependent organ on oxygen for its normal function. Studies of hemodynamics in the human brain are important for understanding mechanisms involved in brain function and the development of diagnostic and treatment strategies in different cerebrovascular disorders [33]. Determination of a non-destructive method for quantifying 
spatial-temporal response to oxygenation changes in the brain was achieved in the 1960's by studying the fluorescence emission of cell mitochondria of rat brain [40]. Cytochrome components found in the mitochondria has a large absorption band from $390 \mathrm{~nm}$ to $500 \mathrm{~nm}$, and oxidation of these components allows for these components to be highly susceptible to fluorescence emission in exercised tissue [40]. A section of the animal's skull was removed and measurements were made directly on the cortex. They were able to quantify tissue hypoxia through corresponding changes in hemoglobin concentration on animal models using spectrofluorometers [40]. The use of NIR wavelengths was shown in the 1930's by Kramer [41], to produce greater tissue transmission in studies through the animal skull, and was applied by Jobsis [42] to neonatal head in hypoxic studies.

Many NIRS studies have demonstrated that hemodynamic changes in relation to functional brain activity can be assessed non-invasively in adult human subjects $[18,30,43,44]$. There are two different signals related to brain activity that can be monitored using NIRS. One of the most promising attribute of NIRS is the ability to measure fast changes in optical properties of cerebral tissue $[44,45,46]$. A neuronal signal caused by a change in the optical scattering properties of the neurons upon activation and occurs within milliseconds of the onset of stimulation [46]. In few seconds after the beginning of activation, the hemodynamic response of the cortex can be measured as the increasing oxygen consumption in the activated area results in an increase in blood perfusion which leads to changes in hemoglobin concentration and blood oxygenation levels and hence a change in the tissues optical properties $[43,44,45]$. Using NIRS to monitor brain activity was shown to be an effective addition to electro-encephalography 
(EEG), magneto-encephalography (MEG), functional magnetic resonance imaging (fMRI) and positron emission tomography (PET) [45].

\section{5 - Current Alternative Techniques Used for Cerebral Imaging}

There are several neuroimaging techniques available for examining functional brain activity. Historically, EEG was the first to appear followed by other modalities such as MEG, PET and more recently fMRI. These can be categoized in terms of whether they provide direct or indiect information about neuronal function. EEG and MEG are the direct methods, wereas PET and fMIR are indirect methods that are in general called hemodynamic techniques because they investigate neural activity by measuring hemodynamic changes [47]. A less known technique for monitoring brain funtion is Diffuse Optical Tomography (DOT) which is a multichannel form of NIRS. Each of these techniques has its own distinct advantages and disadvantages.

\subsection{1 - Electro- and Magneto- encephalography}

Electro-encephalography provides direct measurements of neuronal activation. It records electric potentials on the scalp produced by the firing of neurons within the brain [48]. The main diagnostic application of EEG is epilepsy, as epileptic activity can create abnormalities that are easily determined using EEG. In MEG, the same magnetic fields produced in EEG are detected outside the head with SQUID (Superconducting Quantum Interference Device) magnetometers [49]. The magnetic fields are produced by the electrical activity in the brain. There are two processes that can be studied by means of MEG [49]. The first is evoked responses which sensory stimuli are presented to the test subject and the magnetic field created, is recorded at 
several locations on the head. Second is spontaneous brain activity resulting from localized events in the brain such as epileptic activity [49]. Presently, MEG is considered to have much better spatial resolution than EEG.

\subsection{2 - Positron Emission Tomography}

Positron Emission Tomography (PET) is a nuclear medical imaging technique that provides 3-dimensional (3D) images of the functional processes in the body [50]. A short-lived positron-emitting radioactive tracer isotope is injected into the body and a PET scan is taken which is converted to a complex picture of the brain function of the patient. The system detects pairs of gamma rays emitted by the positron emission decay of the radioisotope. Once the radioisotope is injected, it undergoes beta decay where a position is emitted [51]. The positron travels till it encounters an electron and annihilation occurs in which two gamma photons are created that travel in opposite directions. This pair of gamma photons is detected by the photodetector array, and images of tracer concentration in 3D within the body are reconstructed by computer analysis. PET measures the concentration of the tracer. The tracer is either biologically active or chemically bound to a biologically active molecule [52]. Therefore, PET measures what the active molecule is sensitive to. The specific tracer given to the patient is dependent on what brain function is being monitored and each tracer closely resembles a natural molecule utilized by the body [52]. For example, 2-fluoro-2-deoxy-D-glucose is similar to glucose with the addition of the radioisotope fluorine. The gamma rays detected from the positron emission from fluorine by the PET scanner shows in fine detail the metabolism of glucose in the brain [52]. PET can also measure regional cerebral blood flow and cerebral metabolic rate of oxygen. Oxygen-15 is used as the tracer to label oxygen gas for the 
measurement of oxygen metabolism in order to study these cerebral functions [53]. Disadvantages of PET include: continuous monitoring is not possible due to a relatively low resolution time, it is very expensive and the requirement of the application of a radioactive contrast agent makes this imaging technique invasive $[12,54]$.

\subsection{3 - Functional Magnetic Resonance Imaging}

Functional Magnetic Resonance Imaging (fMRI) is a medical imaging technique that measures the hemodynamic response related to brain activity. It uses strong magnetic field to align the nuclear magnetic moments of hydrogen atoms of water found in the tissue [55]. Radio frequency (RF) electromagnetic waves are briefly turned on which cause the protons in the hydrogen atoms to alter their alignment of magnetization relative to the magnetic field. The RF is then turned off and the protons return to their original alignment with the magnetic field. The alignment changes create a signal with a specific Larmor frequency of the magnetic resonance and this RF field is detected by the scanner [55]. Increases in neural activity causes changes in blood volume and oxygenation, where an increase in the concentration of HHb will change the magnetic susceptibility [56]. When a brain region is activated, $\mathrm{HbO} 2$ in the region increases beyond the actual oxygen demands, and the associated decrease in $\mathrm{HHb}$ concentration yields the signal detected with blood oxygenation level dependent (BOLD) MIR [57]. fMRI has a high acquisition time (around $50 \mathrm{~ms}$ ) and spatial resolution $(0.5 \mathrm{~cm}$ or better) and absence of any penetration limits [58]. However, changes in $\mathrm{HHb}$ are not measured quantitatively and fMRI does not provide any information on $\mathrm{HbO} 2$ concentrations [57]. fMRI is very sensitive to motion artifacts. It is non-invasive and provides both structural and functional information [12, 
23], but its physiological sensitivity is limited to unquantifiable $\mathrm{HHb}$ concentration changes. It is quite expensive both in terms of equipment and service costs.

\subsection{4-Diffuse Optical Tomography}

Diffuse optical tomography is the only imaging technique that is potentially able to provide simultaneous, noninvasive measurements of neuronal and hemodynamic signals from the brain cortex [31]. Essentially it is a version of NIRS which uses multiple channels where light is shone onto the scalp from multiple sources and detected as it exits the head at different locations. Then through the use of the specific absorption spectra of the main tissue chromophores, DOT can measure local changes in chromophore concentrations. The advantages of DOT over the other previously mentioned imaging modalities are that it has the capability to measure concentrations of oxy- and deoxy- hemoglobin, water, and fat in tissues, and it is the most cost-effective and least invasive method of imaging [48].

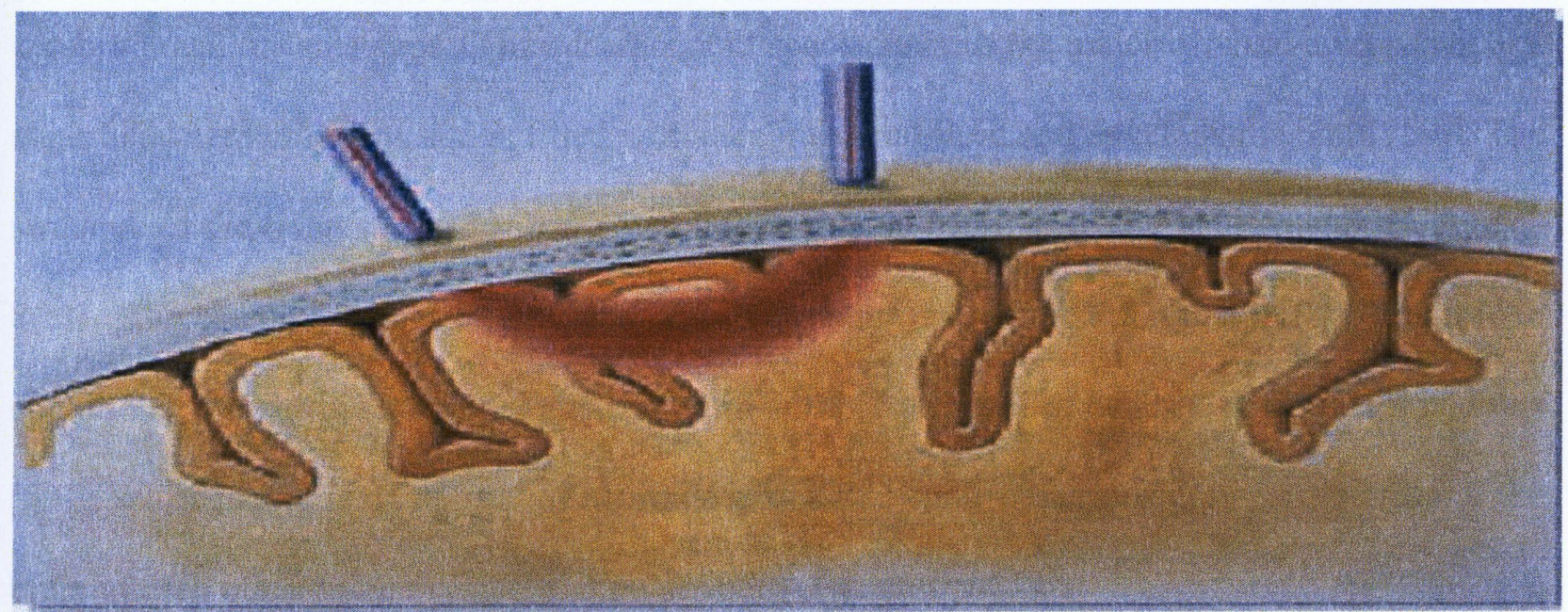

Figure 1-7- How light travels from source to detector when placed on top of the human head. Light is shone onto the scalp and detected as it exits the head, from Izzetoglu et. al. [16]. 


\section{6 - Anatomy and Physiology of the Human Head}

The human head has a complex anatomy with nested structures that intertwine into one another. The human brain is protected by a number of tissue layers that can be seen in Figure 18(a). These layers include the skull, which shields the brain from impacts, the meninges, which consists of three membranes surrounding the brain to shield it from damage by contact with the skull, and the cerebro-spinal fluid (CSF) [59]. CSF circulates through the ventricles and between the pia and arachnoids matter of the meninges. Its purpose is to cushion blows to the head and reduce pressure at the base of the brain [59]. The three layers of the meninges consist of the dura mater, the arachnoid mater and the pia mater. The outer layer is the dura mater which is a tough layer of collagenous connective tissue. The middle layer consists of the arachnoid mater that is a translucent membrane through which the subarachnoid space, cerebrum and the blood vessels are found. Lastly, the inner layer is composed of pia mater which directly supplies blood for the cerebrum and lines its gaps. The subarachnoid space contains the major cerebral arteries and the superficial cerebral veins and is filled with cerebralspinal fluid and envelops the brain [59]. The structure of the brain can be broken down into two main parts: grey and white matter (Figure 18(b)). The cerebral cortex, or gray matter, is a structure within the brain that has the key role in cognition. It is the outer layer of the cerebrum and is composed of dendrites of cerebral neurons [59]. White matter is composed of neuronal axons that connect various gray matter areas of the brain to each other and carry nerve impulses between neurons [59]. 


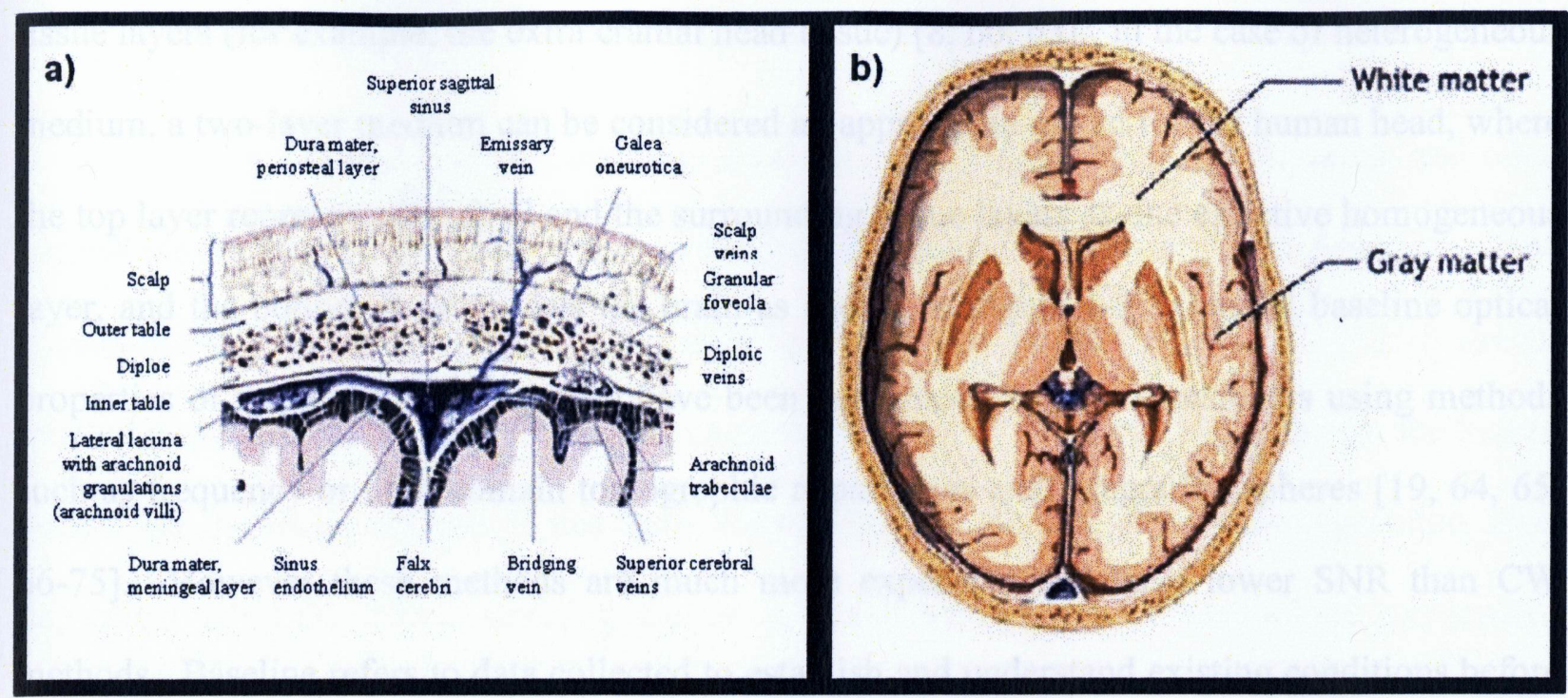

Figure 1-8- a) The anatomy of the human head which demonstrates the many layers which surround the brain. b) The brain itself is composed of white and gray matter. From Schünke et. al [59].

\section{7-NIR Spectroscopy and Current Two-Layered Models of the Human Head}

As described in the previous section, the human head is a very complex structure, where there are many layers which surround the brain. Most of previous cerebral NIRS studies neglected the influence of the extracerebral tissue on brain signals. However, it is obvious that extracerebral tissues indeed should strongly affect the behaviour of light $[60,61]$. Therefore, when performing cerebral hemodynamic measurements using NIRS, it is important to consider the influence of layers surrounding the brain. The thickness of the layers between the surface of the human head and the brain was determined by $\mathrm{Li}$ et al. [62] to be approximately $10-15 \mathrm{~mm}$ for an average adult human head [62]. Ongoing research is being preformed to develop NIR imaging methods for heterogeneous tissue. The approach has been to model the head as a layered problem, and the use of the diffusion approximation to the radiative transfer theory to determine non-invasively the optical properties of internal tissue in the human brain, through the external 
tissue layers (for example, the extra cranial head tissue) $[8,60,63]$. In the case of heterogeneous medium, a two-layer medium can be considered an appropriate model for the human head, where the top layer represents the skull and the surrounding tissue layers as one effective homogeneous layer, and the bottom layer models the brain as shown in Figure 1-9. General baseline optical properties of the human head in vivo have been determined by past researchers using methods such as frequency or time domain tomographic apparatuses and integrating spheres $[19,64,65$, 66-75]. However these methods are much more expensive and have lower SNR than CW methods. Baseline refers to data collected to establish and understand existing conditions before any experimental manipulation occurs. A compiled a list of tissue baseline optical properties may be found elsewhere [66]. It presents data that has been produced by many groups over the years, both experimental and compiled in vivo and in vitro optical properties [19, 64, 65, 66-75]. There is a large variation among reported values due to the different methods and the conditions of measurements applied.

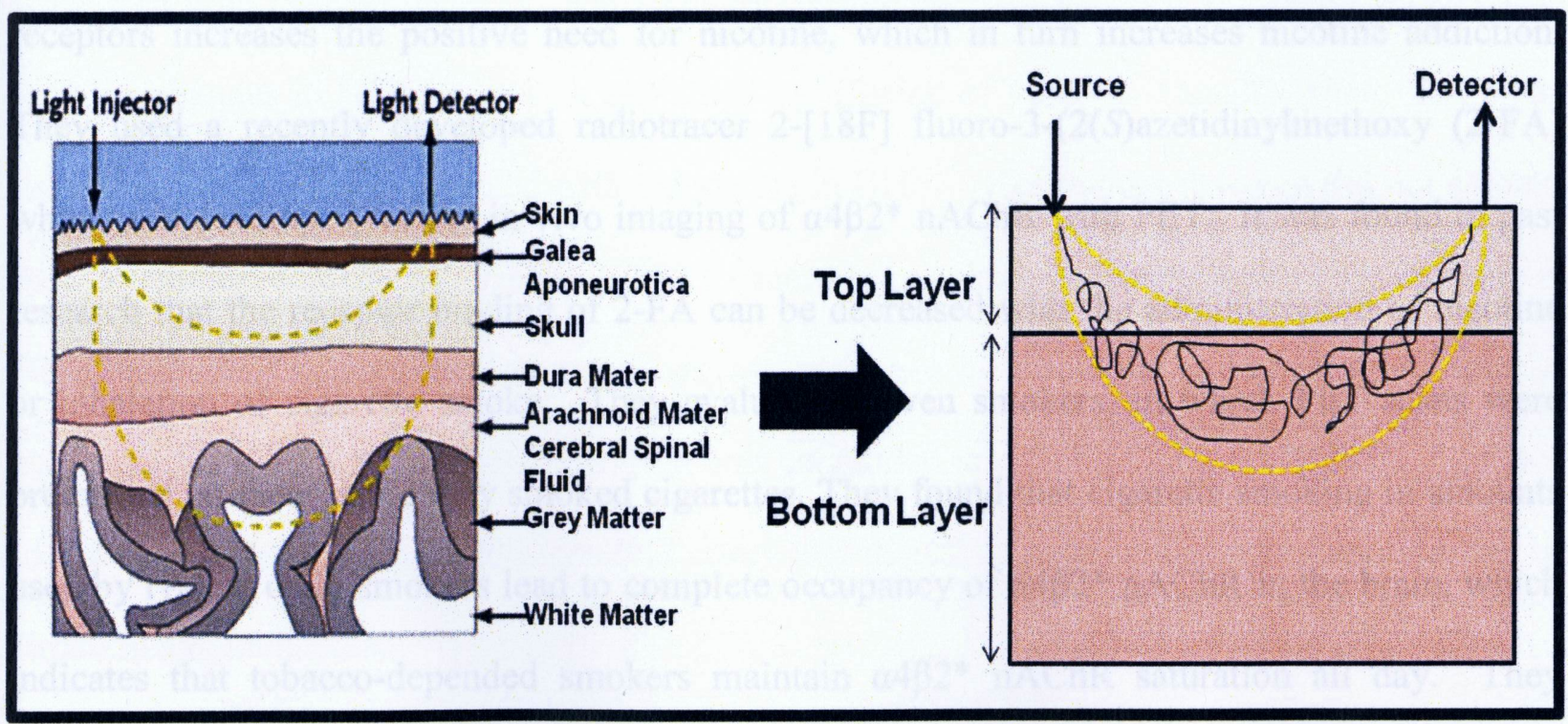

Figure 1-9- Two-layer medium approximate model of the human head, where the top layer represents the scull and the surrounding tissue layers as one homogeneous effective layer, and the bottom layer models the brain. 


\section{8 - Naturally Administered Smoke and Cerebral Hemodynamics}

It is well known that that cigarette smoking causes lung cancer, emphysema, and other serious diseases [76]. Smoking is one of the leading causes of death in North America, where approximately 5 million Canadians alone ages 15 years and older smoke $[77,78]$. The chemicals inhaled during the smoking of a cigarette cause a number of physiological changes in the human body. Of particular interest it has been shown through the use of blood-oxygenation level dependent (BOLD) fMRI, smoking causes a global change in the amount of oxygenated hemoglobin found in the brain $[18,79,80]$. The disadvantages of these studies were that they used direct nicotine administration into test subjects rather than natural cigarette smoking, and fMRI provides only a qualitative indicator of hemodynamic changes. Reports have shown that smoking affected cerebral oxygenation levels [81]. Brody et. al [82], studied the effect of cigarette smoking on the brain by examining nicotinic acetylcholine receptors $(\alpha 4 \beta 2 * \mathrm{nAChR})$ occupancy in tobacco-dependent smokers using PET. Nicotine with nicotinic acetylcholine receptors increases the positive need for nicotine, which in turn increases nicotine addiction. They used a recently developed radiotracer 2-[18F] fluoro-3-(2(S)azetidinylmethoxy (2-FA) which was developed for the in vivo imaging of $\alpha 4 \beta 2 * \mathrm{nAChR}$ with PET. It was found in past research that the receptor binding of 2-FA can be decreased with the administration of nicotine or inhalation of cigarette smoke. They evaluated eleven smokers, in which PET scans were preformed on them while they smoked cigarettes. They found that cigarette smoking in amounts used by typical daily smokers lead to complete occupancy of $\alpha 4 \beta 2 *$ nAChR in the brain, which indicates that tobacco-depended smokers maintain $\alpha 4 \beta 2 * \mathrm{nAChR}$ saturation all day. They concluded that prolong binding of nicotine to $\alpha 4 \beta 2 * \mathrm{nAChR}$ causes these receptors to be desensitized for a long period which suggests alleviation from tobacco withdrawal. Small 
amounts of nicotine do not bind to these receptors for a long period of time, hence causing tobacco cravings much more frequently for those individuals that smoke less often. Siafaka et. al. [79] used NIRS to study long-term effects smoking has on skeletal muscle microcirculation. Vascular responses to brachial artery occlusion measured by NIRS were compared for groups of smokers and non-smokers. The conclusion of the study was that vascular microcirculation in skeletal muscles in smokers is significantly impaired. Birkner et. al. [81] examined if cigarette smoking causes an increase in cerebral blood flow velocity (CBFV) and reduce vasomotor reactivity using doppler sonograph and NIRS. They found that smoking significantly increased $\mathrm{CBFV}$ in the middle cerebral artery compared to baseline values. As well, $\mathrm{HbO} 2$ and total hemoglobin concentrations rose, while $\mathrm{HHb}$ concentration and tissue oxygenation index (TOI) did not change for the entire protocol. During smoking there was an increase in mean arterial blood pressure, heart rate and skin blood flow. After smoking CBFV remained elevated while $\mathrm{HbO} 2$ concentration levels returned to baseline. Changes in TOI during and after smoking were correlated with changes in $\mathrm{HbO} 2$ and $\mathrm{HHb}$ concentrations.' An increase in $\mathrm{HbO} 2$ concentration with no changes in $\mathrm{HHb}$ cannot be explained by dilation of vessels. Usually when there is an increase in $\mathrm{HbO} 2$, it is paralleled with a decrease in $\mathrm{HHb}$. Hence their research did not support their hypothesis that the increase in CBFVs caused by smoking, accompanied by the increase in $\mathrm{HbO} 2$ and total hemoglobin concentrations, are explained by the constriction of cerebral arterial accompanied by arteriolar dilation [81]. It was concluded that the increase in CBFV may be due to the constriction of the middle cerebral artery and therefore contribute to the increased risk of stroke in smokers.

Many obstacles arise when determining the effects naturally administered smoke has on cerebral hemodynamics using the available types of imaging modalities. This is due to a number 
of factors such as safety, discomfort, cigarette odors and residue, and the technical aspects of drug delivery [81]: Smoke is composed of hundreds of chemicals and in order to study the effects these chemicals have on cerebral hemodynamics, as opposed to direct administration of nicotine, smoke must be administered naturally during experiments.

A mobile NIR sensor easily allows for the effects of naturally administered smoke on cerebral hemodynamics to be determined on test subjects in their natural environments, rather than in a magnetic resonance scanning room. In past research done on the human head to determine the effect smoking has on cerebral hemodynamics, it was assumed as one homogeneous medium. Surrounding layers of the brain were not taken into consideration, which as mentioned previously does influence the behavior of NIR light. Hence applying a two-layered approach to the human head can quantify accurately changes in cerebral hemodynamics of the brain during smoking.

\section{9 - Thesis Organization and Objectives}

The goal of this research is the development of a method to quantify accurately and noninvasively the hemodynamic changes in the brain by accounting for the presence of the extracerebral tissues and using broadband CW NIRS. The approach is based on the two-layer model of the human head where the top layer represents extra-cerebral tissue, and the bottom layer represents the brain. Recovery of optical properties for the different layers can quantify accurately both $\mathrm{HbO} 2$ and $\mathrm{HHb}$ concentrations in the brain. It is hypothesized that cerebral hemodynamics can be quantified accurately by determining the optical properties of a two- 
layered model of the human head using broadband optical spectroscopy of tissue over the NIR spectrum.

The method consists of two steps shown in the flow chart found in Figure 1-10. The first step is to measure the effective absorption and scattering parameters of the two-layered model of the human head by performing a multi-distance fit of the spectral differential of the absorbance in the $650-1000 \mathrm{~nm}$ waveband. The second step is to quantify changes in the optical properties of the brain through the external tissue layer using the DPF values for the cerebral and extracerebral components calculated using the optical properties obtained at the first step.

There are three main experiments presented in this thesis: 1) Phantom validation, 2) In vivo baseline measurements, and 3) In-vivo measurements of hemodynamics during smoking. Before performing measurements on test subjects the approach was validated using phantoms with known optical properties. After the method was proved to provide accurate optical properties for each layer, in vivo baseline measurement were performed on adult humans. Lastly, in-vivo measurements were performed using the mobile NIR to determine the effect of smoking on temporal changes in oxyhemoglobin and deoxyhemoglobin concentrations. 


\section{Part I:}

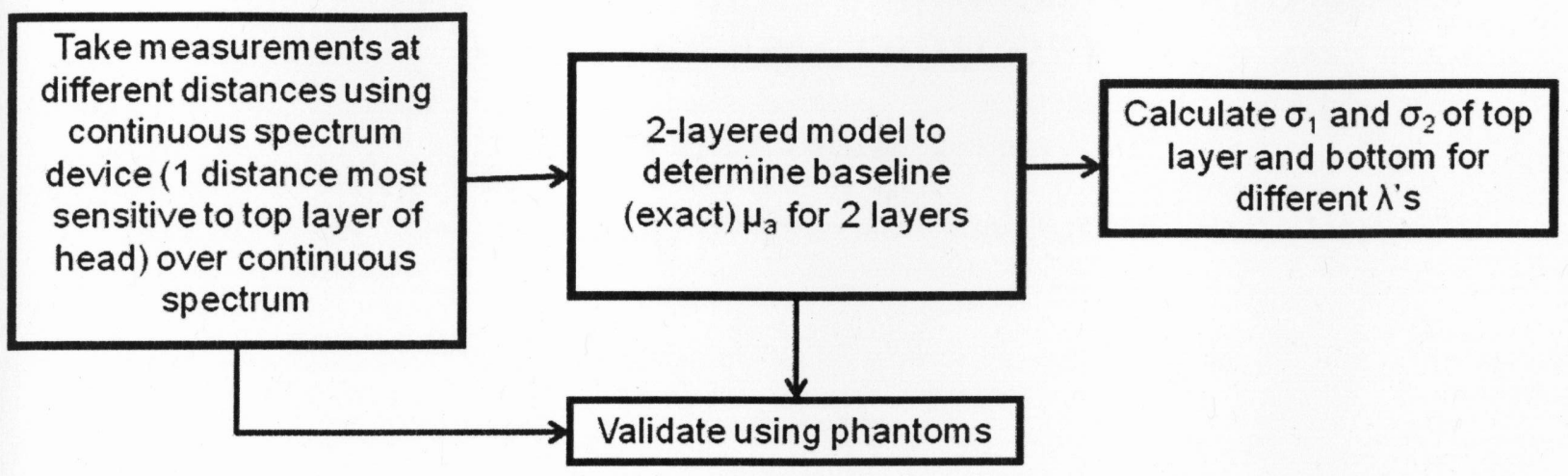

\section{Part II:}

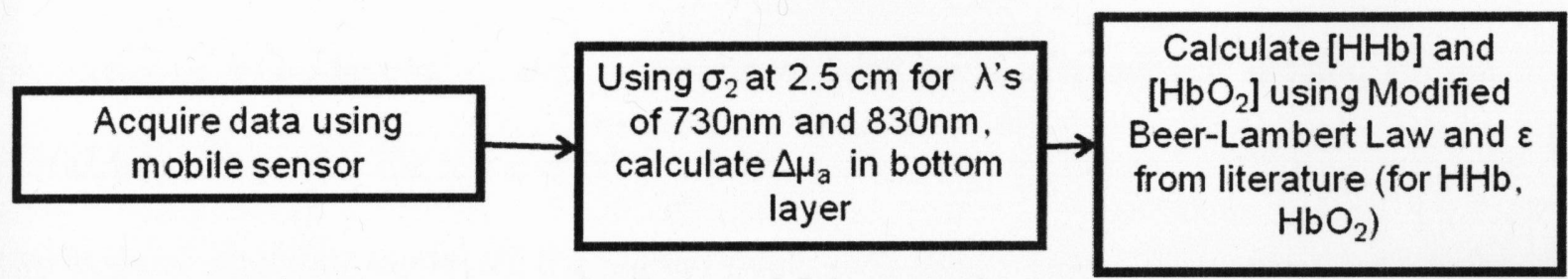

Figure 1-10- Schematic diagram of methodology organization. Approach is based on three main experiments: 1) Phantom validation 2) In vivo baseline measurements and 3) Changes in cerebral hemodynamic.

Chapter 2 presents a journal manuscript on the boradband two-layered model approach and baseline measurements. Chapter 3 explains the methodology of calculating the DPF and mobile sensor data, Chapter 4 presents the results and discussion of DPF and mobile NIRS results. The final Chapter presents conclusions and future work ideas. 
2-Journal Manuscript 


\title{
MEASUREMENT OF THE OPTICAL PROPERTIES OF A TWO-LAYER MODEL OF THE HUMAN HEAD USING BROADBAND NEAR- INFRARED SPECTROSCOPY
}

\author{
Olivia Pucci and Vladislav Toronov \\ Dept. of Physics, Ryerson University, 350 Victoria St., Toronto, ON CANADA M5B 2K3
}

\begin{abstract}
We present the development of a continuous-wave method to quantify accurately the optical properties of a two-layered model of the human head using a broadband spectral approach. In particular we focus on the reconstruction of the absolute absorption and scattering properties of a two-layered phantom model of the human head with steady-state multi-distance measurements by performing differential fit analysis of the NIR reflectance spectrum between $700 \mathrm{~nm}$ and 1000 nm. The two-layered model approximation was fitted to experimental broadband absorbance measurements obtained from two-layered phantoms with known optical properties. Our results demonstrated that our method was able to determine the optical properties of the lower layer with minimal error at specific source-detector distances. We also present our preliminary results on the non-invasive measurement of the optical properties of the adult human brain in a twolayer approximation. Our results suggest that broadband NIRS can detect cortical hemodynamic disorders in humans.
\end{abstract}

\section{INTRODUCTION}

Studies of the human cerebral hemodynamics are important for understanding the mechanisms of brain function and for the development of diagnostic and treatment strategies in different cerebrovascular 
disorders. In recent years, studies have shown the feasibility to measure hemodynamic changes associated with functional brain activity non-invasively using near-infrared spectroscopy (NIRS) [1-4]. However, methods for non-invasive NIRS quantification of the absolute physiological parameters of the brain still need to be furthered.

Most applications of NIRS have used the diffusion approximation to the radiative transfer model to predict light propagation in tissue, which in turn can be used to examine physiological parameters. However, the drawback of many studies has been an explicit or implicit assumption that the tissue was homogeneous [5]. In actuality, the brain is a complex structure surrounded by many layers such as the skull, dura mater and cerebrospinal fluid [6]. Therefore, when performing cerebral measurements using NIRS, it is important to account for the influence of the surrounding layers of the brain on light transport $[7,8]$.

Some cerebral NIRS studies have tried to determine non-invasively the optical properties of the human brain through the external tissue layers using frequency or time domain systems [9-12]. In particular, it was shown that the measurements of optical properties of a two-layered turbid medium with a thin $(1-2 \mathrm{~mm})$ top layer is possible $[9,10]$. However the time- and frequency-domain methods have a drawback of high equipment costs at relatively low signal-to-noise ratio.

Although the separation of the absorption and scattering properties of even homogeneous turbid medium at a particular wavelength is impossible using the continuous-wave $(\mathrm{CW})$ approach, it was shown that using the quasi-continuous spectral data one can quantify optical properties of a homogeneous medium at least in the vicinity of distinct spectral features, such as the water absorption peaks [13]. In this study, we present the development of a method to quantify the optical properties of a two-layered model of the human head using a CW broadband approach. In particular we focus on the reconstruction of the absorption and scattering properties of a two-layered model of the human head with steady-state multi-distance measurements by performing differential fit analysis of the NIR reflectance spectrum between $700 \mathrm{~nm}$ and $1000 \mathrm{~nm}$. We assume a fixed thickness for the top layer of about $15 \mathrm{~mm}$ and 
perform multi-distance $\mathrm{CW}$ broadband measurements to allow for the interrogation of different layers by varying source-detector (SD) distances. As an experimental validation of our method, we reconstruct the optical properties of a two-layer phantom from the absorbance measured at varying SD distance on the surface of the phantoms with known optical properties of layers. We also present our preliminary results of the in-vivo depth-resolved measurements of the optical properties of adult human subjects in a twolayer approximation.

\section{MATERIALS AND METHODS}

\subsection{Broadband Optical Spectroscopy of Tissue: The Light Source and Detector}

The NIR light source used in the experimental set up was a stabilized halogen fan-cooled AvaLight-HAL Tungsten Halogen Light Source (Avantes Inc., Broomfield, CO) with an adjustable focusing connector in order to maximize light coupling into the source fiber. The optical fiber bundle with a diameter of $3 \mathrm{~mm}$ and a $400 \mu \mathrm{m}$ diameter single fiber was used to transmit the light from the source to the medium. The optical power at the source fiber tip was about $5 \mathrm{~mW}$.

The detector fiber was connected to a QE65000 Scientific-grade Spectrometer (Ocean Optics, Dunedin, FL), which had a spectral range between 650 and $1100 \mathrm{~nm}$. The detector was cooled down to $15^{\circ} \mathrm{C}$ to reduce the dark noise. The spectrometer had a dynamic range of $25000: 1$ and a signal-to-noise ratio of 1000:1. The optical fibers collected and transmitted the light from the sample to the spectrometer. The spectrometer output was digitized using the Spectral Suite software (Ocean Optics, Dunedin, FL). The absorbance spectra were calculated using the equation:

$$
A_{\lambda}=-\log _{10}\left[\frac{S_{\lambda}-D N_{\lambda}}{R_{\lambda}-D N_{\lambda}}\right]
$$


Where $D N_{\lambda}$ was the dark spectrum, $R_{\lambda}$ was the reference intensity, and $S_{\lambda}$ was the sample intensity. Measurements were taken with the following acquisition parameters: integration time of 5 seconds, 20 scans averaged and the 5-point moving averaging to smooth the spectra.

\subsection{Data Processing}

\subsubsection{Broadband Approach to Forward Modeling}

Tissue is a highly scattering medium where the scattering coefficient is approximately hundreds of times larger than the absorption coefficient. This is the case of validity of the diffusion approximation to the radiative transfer model [15]. Although the mammal head is a complex structure containing many tissue layers, as a simplest modification to the homogeneous model, we modeled the human head as a semi-infinite two-layer medium with the $10-15 \mathrm{~mm}$ thick planar top layer representing the skull and the scalp, and the bottom layer representing the brain. The corresponding solution of the light diffusion equation was first obtained by Kienle et al. in the form of a Hankel transform [16]:

$$
\Phi(\rho, z)=\frac{1}{2 \pi} \int_{0}^{\infty} \phi(s, z) s J_{0}(s \rho) d s
$$

where,

$\phi(s, z)=\frac{\sinh \left[\alpha_{1}\left(z_{b}+z_{0}\right)\right]}{D_{1} \alpha_{1}} \frac{D_{1} \alpha_{1} \cosh \left[\alpha_{1}(l-z)\right]+D_{2} \alpha_{2} \sinh \left[\alpha_{1}(l-z)\right]}{D_{1} \alpha_{1} \cosh \left[\alpha_{1}\left(l+z_{b}\right)\right]+D_{2} \alpha_{2} \sinh \left[\alpha_{1}\left(l+z_{b}\right)\right]}-\frac{\sinh \left[\alpha_{1}\left(z_{0}-z\right)\right]}{D_{1} \alpha_{1}}$

Here $\Phi$ represents the fluence rate in units of $\mathrm{W} / \mathrm{m}^{2}, \rho$ is the source-detector distance, $l$ is the thickness of the top layer and $D_{1}$ and $D_{2}$ are the diffusion constants where $\mathrm{D}=\left(3 \mu_{\mathrm{a}}+3 \mu_{\mathrm{s}}\right)^{-1}$ in units of $\mathrm{cm}$. For a given layer $\alpha_{i}^{2}=\left(D_{i} s^{2}+\mu_{a i}+\mathrm{j} \omega \mathrm{c}_{\mathrm{i}}\right) / D_{i}$ where $s$ is the dummy variable in the Hankel transform image space, $\mu_{a}$ is the absorption coefficient in $\mathrm{cm}^{-1}$ of the top and bottom layers respectively, $c_{i}$ is the speed of light in both 
layers of the medium and $\omega$ is the circular amplitude modulation frequency of the incident light. $z_{0}$ and $z_{b}$ are the approximate boundary parameters defined by the expressions $z_{0}=3 \mathrm{D}$ and $z_{b}=2 \mathrm{D}, \mathrm{z}$ is the depth of the scattered beam in the upper layer, and $\mu_{s}{ }^{\prime}$ represents the reduced scattering coefficient. Equation (3) can be applied to any source modulation type, however we used continuous wave and thus the term $j \omega c_{i}$ was zero. This model was depended on six parameters, which were the source-detector distance (SD), the absorption and reduced scattering coefficients of the top and bottom layers, and the top layer thickness. We have developed a special numeric procedure that allowed for fast and accurate calculations using Equations (2) and (3), which was necessary at top layer thickness more than $1 \mathrm{~cm}$ [20]. In order to further speed up the algorithm we computed the two-layer medium reflectance in a wide range of all parameters mentioned previously.

\subsubsection{Regression Analysis}

To model the absorption coefficients of the animal head layers, a combination of known absorption spectra of water and fat and the specific absorption spectra of deoxyhemoglobin $(\mathrm{HHb})$ and oxyhemoglobin (HbO2) were used as follows:

$$
\mu_{a_{1,2}}(\lambda)=\left[H b O_{2}\right]_{1,2} \varepsilon(\lambda)_{H b O_{2}}+[H H b]_{1,2} \varepsilon(\lambda)_{H H b}+\left(\% F C_{H_{2} O}\right) \mu_{a_{H_{2} O}}+\left(\% F C_{f a t}\right) \mu_{a_{f a t}}
$$

where, the quantities in square brackets represented the concentration of oxy- and deoxyhemoglobin and $\varepsilon(\lambda)$ represented their molar extinction respectively. $\mu_{a_{H 2 O}}$ and $\mu_{a_{f a t}}$ were the absorption coefficients of water and fat, and $(\% \mathrm{FC})$ was their volume fraction.

Similarly, the absorption coefficient of the two types of phantom media was modeled as:

$$
\mu_{a_{1,2}}(\lambda)=\mu_{a_{\text {silicone }}}+[C B]_{1,2} \varepsilon(\lambda)_{C B}
$$


Where $\mu_{a_{\text {Silicone }}}$ was the silicone absorption coefficient, the quantity in square brackets was the concentration of carbon black and $\varepsilon(\lambda)_{C B}$ was its molar extinction coefficient. $\varepsilon(\lambda)$ of each specie was found in the literature [17-20]. To model the reduced scattering coefficient spectral dependence we assumed that $\mu_{\mathrm{s}}^{\prime}$ as a function of $\lambda$ obeys the power law [20]:

$$
\mu_{s}^{\prime}(\lambda)=A \lambda^{-\alpha}
$$

with specific values of $A$ and $\alpha$ for each layer.

At the initial step, the measurement at a short SD distance $(10-20 \mathrm{~mm})$ most sensitive to the top layer was used to determine the optical properties of the top layer. Then the measurement at a longer source-detector distance $(25-30 \mathrm{~mm})$ was used to obtain the optical properties of the bottom layer. At both steps the first or second spectral derivative of the experimental absorbance (calculated using Equation (1)) was compared with the derivatives of theoretical absorbance calculated using equations (2)-(6). The use of the absorbance given by Equation (1), which includes the reference signal, allowed for the elimination of instrumental spectral artifacts, and the use of derivatives allowed for the elimination of the uncertain source-medium and detector-medium contact terms and for the magnification of the spectral features.

The two-layer model was applied assuming known optical properties for the top layer and only finding the optical properties of the bottom layer from the differential fits of the theoretical absorbance to the $10 / 20 \mathrm{~mm}, 10 / 30 \mathrm{~mm}$ and $20 / 30 \mathrm{~mm}$ absorbance data. The parameters of the fit were the concentrations of chromophores, $A$, and $\alpha$. To calculate the reflectance as a function of wavelength the six-dimensional matrix of pre-computed reflectance values was used with the interpn Matlab function. Statistical regression analysis was performed using regstats function in Matlab. 


\subsection{Phantoms}

The experimental setup used in our phantom study is displayed in Figure 1. We used four silicone based blocks (ISS, Champaign, IL, USA) with two different sets of tissue-like optical properties. The blocks were comprised mainly of silicone, with small additions of titanium oxide and carbon black (volume fraction less than 1\%). The optical properties at two distinct wavelengths, $690 \mathrm{~nm}$ and $849 \mathrm{~nm}$, were known from frequency-domain measurements. Two blocks were $14 \mathrm{~mm}$ thick and the other two were $100 \mathrm{~mm}$ thick. They were designed to create two-layer phantoms simulating the structure of the head, where the $14 \mathrm{~mm}$ blocks represented the extracerebral and the $100 \mathrm{~mm}$ blocks represented the brain. One type of medium (Type I) had absorption coefficients of $0.202 \mathrm{~cm}^{-1}$ and $0.210 \mathrm{~cm}^{-1}$ and reduced scattering coefficients of $8.7 \mathrm{~cm}^{-1}$ and $7.6 \mathrm{~cm}^{-1}$ at $690 \mathrm{~nm}$ and $849 \mathrm{~nm}$ respectively. The other type of medium (Type II) had optical properties with the following: the absorption coefficients of $0.134 \mathrm{~cm}^{-1}$ and $0.141 \mathrm{~cm}^{-1}$ and the reduced scattering coefficients of $4.2 \mathrm{~cm}^{-1}$ and $4.4 \mathrm{~cm}^{-1}$ at wavelengths of $690 \mathrm{~nm}$ and $849 \mathrm{~nm}$ respectively.

We combined two types of phantom media into two types of two-layer phantoms. The first model had a Type I top layer and Type II bottom layer which created a two-layer phantom model with a bottom layer having lower absorption and scattering than the top layer (Two-Layer I) and vise versa for the second model (Two-Layer II). The source and the detector fibers were always securely attached to the "top" layer. The phantom experiments contained three types of measurements: one with both blocks having the same optical properties, hence representing a homogeneous medium, the other with the "bottom" layer having the optical properties different from the "top" layer, and the third a control measurement with both layers having the same properties in order to ensure that no artifacts were introduced due to switching the bottom layers. Figure 2 shows the Two-Layer I and control absorbance spectra. In both absorbance spectra the same homogeneous medium spectrum (acquired before switching the bottom layer) was used as a reference. The comparison of spectra in Fig. 2 indicates a good spectral sensitivity of the detector to difference in the optical properties of the bottom layer of the phantom. 
For the two-layer model analysis the quantitative optical properties of phantom media, the carbon black extinction coefficient, and the silicon absorption were required in the entire spectral band. Knowing $\mu_{\mathrm{s}}{ }^{\prime}$ at two wavelengths of each phantom, we were able to calculate the parameters $A$ and $\alpha$ for the reduced scattering coefficient in equation (6). In order to measure the optical properties, the absorbance measurements were taken on both $100 \mathrm{~mm}$ blocks. Reference intensities were acquired at the SD distance of $10 \mathrm{~mm}$ and sample intensities at distances of $20 \mathrm{~mm}$ and $30 \mathrm{~mm}$. A total of 10 measurements were taken for each SD combination and averaged accordingly for the analysis. Then using the measurements of absorbance on homogeneous phantoms at two distances and applying a solution of the diffusion equation for the semi-infinite homogeneous medium [20] (which is identical to the one given by Equations (2) and (3) in the case of homogeneous medium), we were able to solve for the values of the absorption coefficient over the NIR spectrum using fzero function of MATLAB and starting at the wavelengths with known $\mu_{a}$. Reconstructed absorption and reduced scattering spectra for the homogeneous phantoms are shown in Figures 3 and 4 respectively.

Since the absorption due to silicone in both types of phantom materials was the same and the concentrations of carbon black $C_{1}$ and $C_{2}$ were known, the carbon black extinction coefficient could be found using the equation:

$$
\mu_{a 1}-\mu_{a 2}=\left(C_{1}-C_{2}\right) \varepsilon_{C B}(\lambda)
$$

The absorption coefficient of silicone (Figure 5) was found by subtracting the contribution of carbon black from the total absorption coefficient of the material:

$$
\mu_{\text {asil }}=\mu_{a 1}-C_{1} \varepsilon_{C B}(\lambda)
$$


Once this was determined the molar extinction of carbon black was calculated (Figure 5) using equation (5). The two-layer model was applied assuming the top layer optical properties known and only finding the optical properties of the bottom layer for the fitting of the theoretical absorbance to the $10 / 20$ $\mathrm{mm}, 10 / 30 \mathrm{~mm}$ and 20/30 $\mathrm{mm}$ absorbance data.

\subsection{Human Subject Procedures}

A total of 10 measurements were performed on adult subjects between the ages of 19 and 51 . After informing each test subject of the nature of the study, each gave their consent. The protocol was approved by the Ryerson University Research Ethics Board (file number REB 2008-003). The human subject was then placed on comfortable seating. To avoid the sinuses the optical sensor was always attached to the top of the forehead, using a sterile roller bandage. This was to ensure that the sensor remained as stable as possible to the subjects head, allowing for minimal movement disturbance. Ten sets of data were acquired at each SD distance $(11$ and $25 \mathrm{~mm}$ ) at different locations (left to right) on the forehead of each subject in the dark room with the same acquisition parameters as described for phantoms. Spectra for the same distances were averaged excluding the outliers.

\section{RESULTS}

\subsection{Phantoms}

In order to determine the ability of our method to yield the optical properties of turbid media, we first applied it to the data acquired on each separate homogeneous phantom. These values are given in Table 1. For both homogeneous phantoms, the errors in the recovered carbon black concentration values were within $4 \%$ and the corresponding $\mathrm{R}^{2}$ values were above 0.9 . 
During our data analysis, we observed that our fitting was highly sensitive to the silicone component in the phantoms, having distinct absorption spectral features at the wavelength range of 730 $760 \mathrm{~nm}$ and $890-940 \mathrm{~nm}$. The differential absorption spectrum values of carbon black were low in these areas and hence very hard to fit over the parts of the spectrum where the spectral features of silicone had a greater influence over those of carbon black. In Figure 6 we present the sensitivity of the theoretical differential absorbance to changes in the bottom layer carbon black concentration (at constant scattering), for our two-layer phantom model over the entire NIR spectrum. Each curve presents the change in absorbance derivative for a range of concentration values, relative to the actual carbon black concentration of the bottom layer. The range of concentration was set to be $25 \%$ below and above the actual concentration value with iterations of $3 \%$ between the set ranges. One can observe that greater changes occurred between the wavelength range of 780-890nm. Using this specific wavelength range in our two-layered fitting analysis, we were able to obtain the best estimates of the optical properties of the bottom layer. Figure 7 represents an example of the first differential absorbance fit for the Two-Layer I phantom. For the Two-Layer I phantom, the best estimate of the optical properties of the bottom layer was obtained using a SD distance combination of $20 / 30 \mathrm{~mm}$ (see Table 1). Although the $\mathrm{R}^{2}$ value of 0.8491 corresponding to Figure 7 was moderate, the recovered optical property values present in Table 1 were very close to the actual optical properties of the bottom phantom. In particular, the error in the concentration of carbon black was as low as $0.0862 \%$. The SD distance combination of $10 / 20 \mathrm{~mm}$ and 10/30 $\mathrm{mm}$ gave a higher error in recovered optical properties for this phantom. For our second twolayered phantom model, Two-Layered II, the recovered optical property values were very close to the actual optical properties for the bottom layer with a percent error of $2.60 \%$ for its concentration of carbon black at a SD distance of $10 / 30 \mathrm{~mm}$. At a SD distance combination 10/20 $\mathrm{mm}$, the linear regression yielded a low $\mathrm{R}^{2}$ value of 0.7038 and a much higher concentration of carbon black than the actual one. For measurements taken on this phantom (Two-Layer II) at a SD distance of $20 / 30 \mathrm{~mm}$, a $\mathrm{R}^{2}$ value 0.4471 , and a high concentration of carbon black compared to the actual concentration were found. For all 
phantoms and all SD distance combinations, the errors in the recovered values of the reduced scattering coefficients were below $10 \%$.

Figure 8 presents a comparison of the reconstructed absorption spectrum of the Type II bottom layer of the Two-Layered I phantom with the absorption spectrum obtained from the measurements on the homogeneous Type II medium. We observe very little difference between the two absorption coefficient spectra, in which they have an $\mathrm{R}^{2}$ value of 0.9929 . A comparison between the reconstructed absorption spectrum of the Type I bottom layer of the Two-Layered II phantom with the absorption spectrum obtained from measurements on the homogeneous Type I medium is found in Figure 9. An $\mathrm{R}^{2}$ value of 0.9911 was determined. Therefore, we can conclude that the reconstruction of the bottom layer phantom optical properties in our two-layered models proved to be successful.

\subsection{Measurements on Human Forehead}

Since the main goal of this paper is to show the validity of our broadband multilayer approach in the phantom study, we include only a brief description of human study results. The detailed report on human studies will be published elsewhere. Our first idea was to follow exactly the approach developed during our phantom studies, namely to acquire spectra at short $(10 \mathrm{~mm})$ and long $(25 \mathrm{~mm}) \mathrm{SD}$ distances on the forehead, to obtain the "top" layer optical properties from the short distance measurement, and then to reconstruct the optical properties of the "bottom layer" using the short-distance measurement as a reference and assuming the thickness of the skull to be 10-15 mm. However, this approach yielded a poor goodness of fit between the theoretical and experimental differential absorbance and unrealistically high $\left[\mathrm{HbO}_{2}\right]$ in the brain. We supposed that that such a mismatch between the optical properties measured at short and long distance resulted from the optical non-homogeneity of the scalp and skull, and therefore, in order to use the two-layer model, some effective optical properties of the "top" layer must be obtained from a long-distance measurement. Since the "top" layer corresponding to tissue between the brain and the surface was assumed to be quite thick $(10-15 \mathrm{~mm})$ so that most of light energy propagated through 
this effective "top", we decided that a good measure of the effective top layer optical properties can be obtained simply from the homogeneous model fit. The homogeneous model fit (Fig. 10) yielded typical values of $\left[\mathrm{HbO}_{2}\right],[\mathrm{HHb}]$ and water fraction of $10 \mu \mathrm{M}, 5 \mu \mathrm{M}$ and $30 \%$, respectively. The reduced scattering coefficient of the "top" layer at $800 \mathrm{~nm}$ was found to be close to $1.0 \mathrm{~cm}^{-1}$ and $\alpha=5$. Assuming these parameters for the "top" layer of our two-layer model and the water fraction of $80-90 \%$ for the brain [14] we obtained for $\left[\mathrm{HbO}_{2}\right]$ and $[\mathrm{HHb}]$ in the brain the values of the order of $40 \mu \mathrm{M}$ and $15 \mu \mathrm{M}$, respectively. (This corresponds to a tissue oxygen saturation (StO2) of $72 \%$ ). The reduced scattering coefficient of the brain at $800 \mathrm{~nm}$ was found to be close to $6.0 \mathrm{~cm}^{-1}$ and $\alpha=6$.

\section{DISCUSSION}

Other groups have already applied multi-layer approach in biomedical NIRS $[5,7,8,9,13,14]$. In particular, Alexandrakis et al. investigated the possibility of determining the optical properties of a twolayered medium using spatially resolved FD reflectance [13]. They used two phantom models: the first consisted of skin overlying muscle and the other of skin overlying fat, each having varying top-layer thicknesses between $1-4 \mathrm{~mm}$. It was assumed the upper layer thickness was unknown, and they determined the optical parameters in both layers. The Monte Carlo simulation model and the two-layer diffusion solution were fitted to experimental frequency-domain reflectance measurements of their models, and both could determine the optical properties of the lower layers [13]. In their research, the use of FD measurements with light from a $677 \mathrm{~nm}$ laser diode, intensity modulated at $100 \mathrm{~Hz}$, only allowed for the researchers to examine data at a discrete wavelength. In addition, the use of Monte Carlo simulations to determine the optical properties of two layers requires a high amount of computational time [14]. However, here we present a very first study combining multi-layer diffusion theory with the broadband CW type of measurements. 
Unlike previous broadband approaches [14] we use not the second but the first spectral derivative of the absorbance and we fit it using tabulated chromophore spectra and concentrations as the fit parameters. Another new point addressed in this study is the feasibility to reconstruct with a good accuracy the optical properties of the bottom layer covered with a thick top layer $(15 \mathrm{~mm})$, which corresponds to the structure of adult human head.

Our method relies on spectral features of natural tissue chromophores. Although the extinction spectrum of carbon black used in phantoms is relatively smooth, our phantom tests confirm the feasibility of the proposed approach. Since tissue chromophores, especially water and deoxyhemoglobin, exhibit much more pronounced NIR spectral features, we expect our method to be practical in biomedical NIRS. Particular areas of applications can be the detection of cerebral hematoma or hypoxia in adults, children, and neonates.

Our preliminary results on the measurements of the absolute optical properties of the two-layer heterogeneous model of human forehead give reasonable values of oxy- and deoxy-hemoglobin concentrations consistent with previous studies [9, 14]. A decrease in the scattering at longer NIR wavelengths is in qualitative agreement with the theory, but quantitatively it appears to be too steep. This and other issues of the accuracy of the $\mathrm{CW}$ broadband method to measure optical properties of heterogeneous tissue will be addressed in future studies.

\section{CONCLUSION}

We have presented a broadband spectral approach to measure absolute optical properties of heterogeneous tissue with layered architecture. Our approach is based on the diffusion approximation solution for the semi-infinite homogeneous two-layer medium and a least squares fit of the first spectral derivative of the absorbance. We have successfully measured the optical properties of the bottom layers in 
two-layer phantoms with $15 \mathrm{~mm}$ thick top layers. Our preliminary in-vivo results provide reasonable cerebral hemoglobin concentrations and oxygenation in healthy adult humans [9].

\section{REFERENCES}

1. Germon, T.J., A.E. Young, A.R. Manara \& R.J. Nelson. (1995). Extracerebral absorption of near infrared light influences the detection of increased cerebral oxygenation monitored by near infrared spectroscopy. Journal of Neurology, Neurosurgery, and Psychiatry 58, 477-479.

2. Gratton, E., V. Toronov, U. Wolf, M. Wolf \& A. Webb. (2005). Measurement of brain activity by nearinfrared light. Journal of Biomedical Optics 10, 11008.

3. Villringer A. \& Chance B. (1997). Non-invasive optical spectroscopy and imaging of human brain function. Trends Neuroscience 20, 435-442.

4. Toronov, V., A. Webb, J.H. Choi, M. Wolf, A. Michalos, E. Gratton, et al. (2001). Investigation of human brain hemodynamics by simultaneous near-infrared spectroscopy and functional magnetic resonance imaging. Medical Physics 28, 521-527.

5. Kienle, A., M.S. Patterson, N. Dognitz, R. Bays, G. Wagninures \& H. van den Bergh. (1998). Noninvasive Determination of the Optical Properties of Two-Layered Turbid Media. Applied Optics 37, 779-791.

6. Schünke, M., M. Schünke, E. Schulte, U. Schumacher, J. Rude, L.M. Ross, E.D. Lamperti, et al. Head and neuroanatomy. Anonymous In "Thieme atlas of anatomy.". Stuttgart ; New York:Thieme (2007).

7. Li, A., R. Kwong, A. Cerussi, S. Merritt, C. Hayakawa \& B. Tromberg. (2007). Method for recovering quantitative broadband diffuse optical spectra from layered media. Applied Optics 46, 4828-4833.

8. Franceschini, M.A., S. Fantini, L.A. Paunescu, J.S. Maier \& E. Gratton. (1998). Influence of a superficial layer in the quantitative spectroscopic study of strongly scattering media. Applied Optics 37, 7447-7458. 
9. Choi, J., M. Wolf, V. Toronov, U. Wolf, C. Polzonetti, D. Hueber, et al. (2004). Noninvasive determination of the optical properties of adult brain: near-infrared spectroscopy approach. Journal of Biomedical Optics 9, 221-229.

10. Firbank, M., M. Hiraoka, M. Essenpreis, and D. T. Delpy. (1993). Measurement of the optical properties of the skull in the wavelength range 650-950 nm. Phys. Med. Biol. 38, 503-510.

11. Okada, E. \& D.T. Delpy. (2003). Near-infrared light propagation in an adult head model. I. Modeling of low-level scattering in the cerebrospinal fluid layer. Applied Optics 42, 2906-2914.

12. Hunter, R.J., M.S. Patterson, T.J. Farrell \& J.E. Hayward. (2002). Haemoglobin oxygenation of a twolayer tissue-simulating phantom from time-resolved reflectance: effect of top layer thickness. Physics in Medicine and Biology 47, 193-208.

13. Matcher, S.J., Cope, M. , Delpy, D. T. (1993). Use of the water absorption spectrum to quantify tissue chromophore concentration changes in near-infrared spectroscopy. Phys. Med. Biol. 38, 177-196.

14. Alexandrakis, G., D.R. Busch, G.W. Faris \& M.S. Patterson. (2001). Determination of the optical properties of two-layer turbid media by use of a frequeny-domain hybrid Monte Carlo diffusion model. Applied Optics 40, 3810-3821.

15. T. Tarvainen, M. Vauhkonen, V. Kolehmainen, J. Kaipio, J. Heiskala, and S. Arridge, "Modeling Photon Migration in Tissues with the Coupled Radiative Transfer Equation and Diffusion Approximation," in Biomedical Optics, Technical Digest (CD) (Optical Society of America, 2006), paper SH49.

16. Kienle, A., M.S. Patterson, N. Dognitz, R. Bays, G. Wagninures \& H. van den Bergh. (1998). Noninvasive Determination of the Optical Properties of Two-Layered Turbid Media. Applied Optics 37, 779-791.

17. Schmitt, J.M. Optical Measurement of Blood Oxygenation by Implantable Telemetry. Technical Report G558-15, Stanford.

18. Moaveni, M.K. (1970). A multiple scattering field theory applied to whole blood. Ph.D. dissertation, Dept. of Electrical Engineering, University of Washington. 
19. R.L.P. Van Veen, R.L.P, H.J.C.M. Sterenborg, A. Pifferi, A. Torricelli \& R. Cubeddu. Determination of VIS- NIR absorption coefficients of mammalian fat, with time and spatially resolved diffuse reflectance and transmission spectroscopy. OSA Annual BIOMED Topical Meeting, 2004.

20. Palmer, K.F. \& D. Williams. (1974). Optical properties of water in the near infrared. Journal of the Optical Society of America 64, 1107-1110.

21. Toronov, V, O. Pucci \& S. Sharieh. (2008). Spectral and spatial characteristics of the differential pathlengths in non-homogeneous tissues. Proceedings of SPIE 6855,685506-1-7. 


\begin{tabular}{|c|c|c|c|c|c|}
\hline Phantom Model & $\begin{array}{c}\text { Source+Detector } \\
\text { Distance }(\mathrm{mm})\end{array}$ & $\begin{array}{c}\text { Error in [Carbon } \\
\text { Black] (\%) }\end{array}$ & $\alpha$ & $\begin{array}{c}\text { Error in } \mu_{s}^{\prime} \\
(\%)\end{array}$ & $\mathbf{R}^{2}$ \\
\hline \multirow[t]{3}{*}{ Type I } & $10+20$ & 51.3 & 0.9 & 8.76 & 0.8106 \\
\hline & $10+30$ & 13.4 & 1.2 & 7.89 & 0.7907 \\
\hline & $20+30$ & 3.78 & 1 & 5.67 & 0.901 \\
\hline \multirow[t]{3}{*}{ Type II } & $10+20$ & 23.9 & 1.2 & 5.67 & 0.7436 \\
\hline & $10+30$ & 43.7 & 1.5 & 5.67 & 0.7593 \\
\hline & $20+30$ & 0.42 & 1.5 & 7.14 & 0.9541 \\
\hline \multirow[t]{3}{*}{ Two-Layer I } & $10+20$ & 23.9 & 1.2 & 9.1 & 0.859 \\
\hline & $10+30$ & 21.9 & 1.2 & 9.1 & 0.86 \\
\hline & $20+30$ & 0.09 & 1.2 & 9.1 & 0.8491 \\
\hline \multirow[t]{3}{*}{ Two-Layer II } & $10+20$ & 36.8 & 1.3 & 7.89 & 0.7038 \\
\hline & $10+30$ & 2.61 & 1.3 & 7.89 & 0.8395 \\
\hline & $20+30$ & 9.32 & 1.3 & 5.53 & 0.4471 \\
\hline
\end{tabular}

Table 1. Recovered optical properties for homogeneous phantoms with the higher optical properties (Type I) and the phantoms with lower optical properties (Type II), and two-layered model fitting for bottom layer having lower optical properties than top layer (Two-Layer I ) and vise versa for TwoLayered II. Reference intensity was measured at the smaller source/detector distance and sample intensity at the larger distance. 


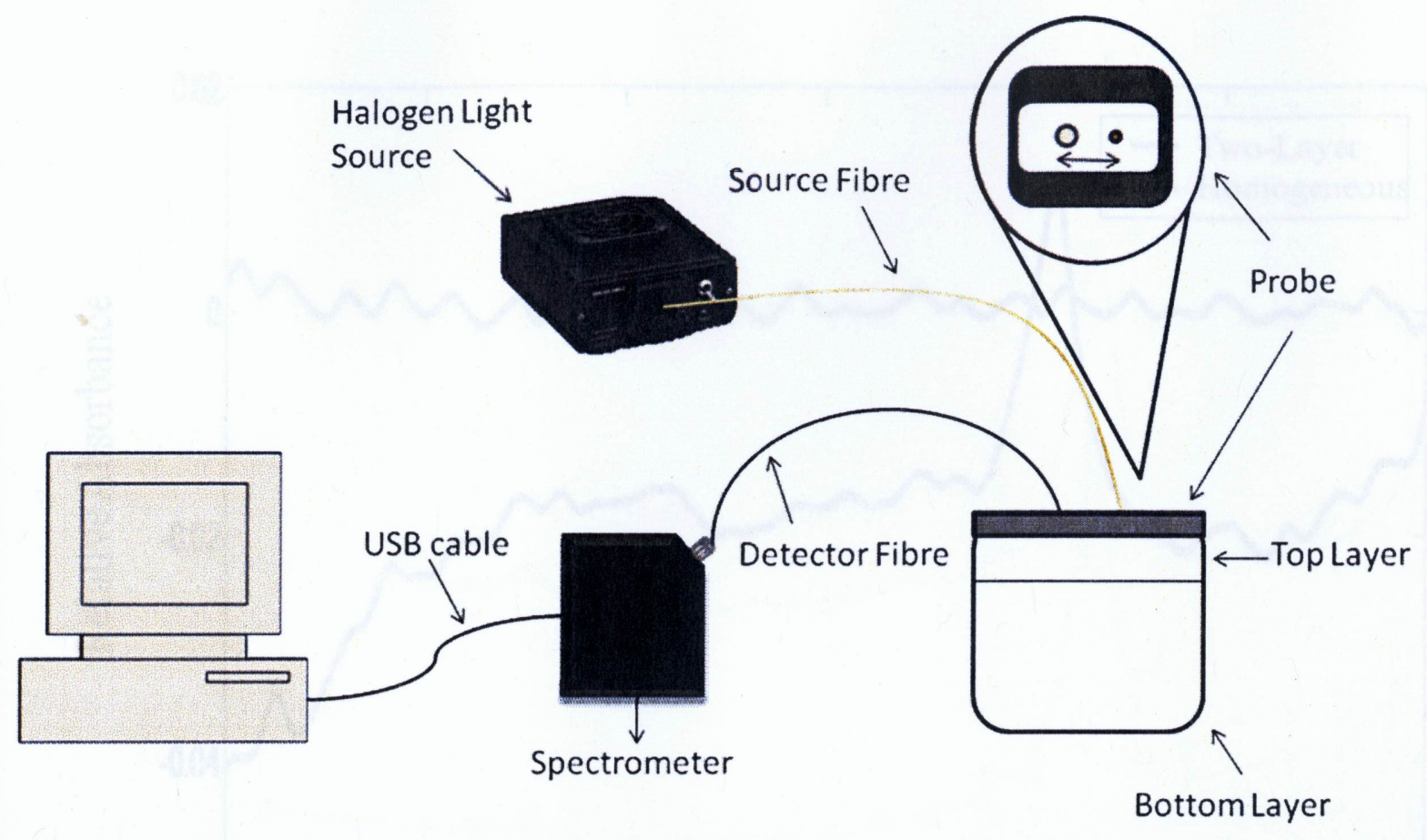

Figure 1. Experimental setup of phantoms. 


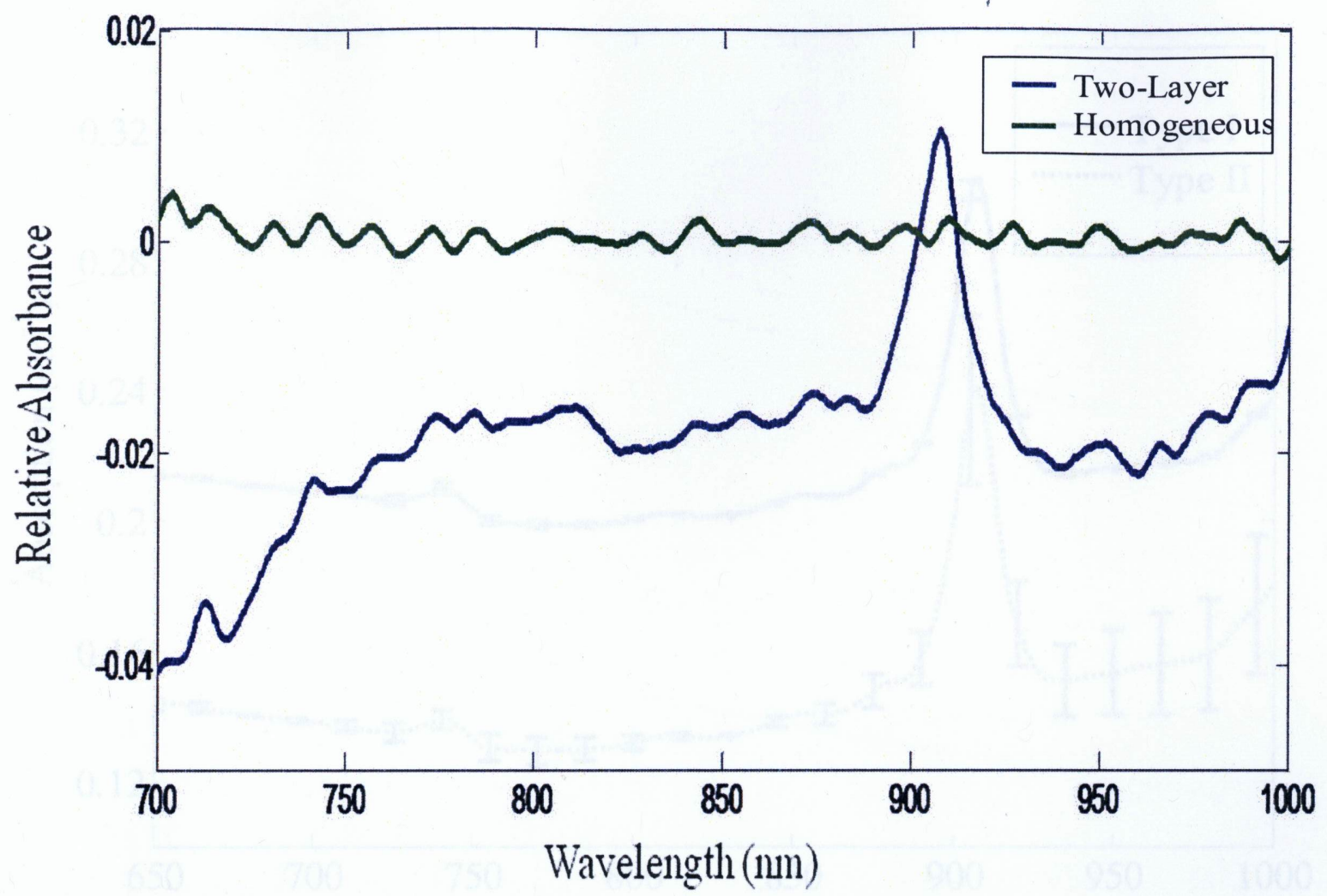

Figure 2. Relative absorbance spectra of phantoms to demonstrate sensitivity of system to bottom layer in a two-layered model. 


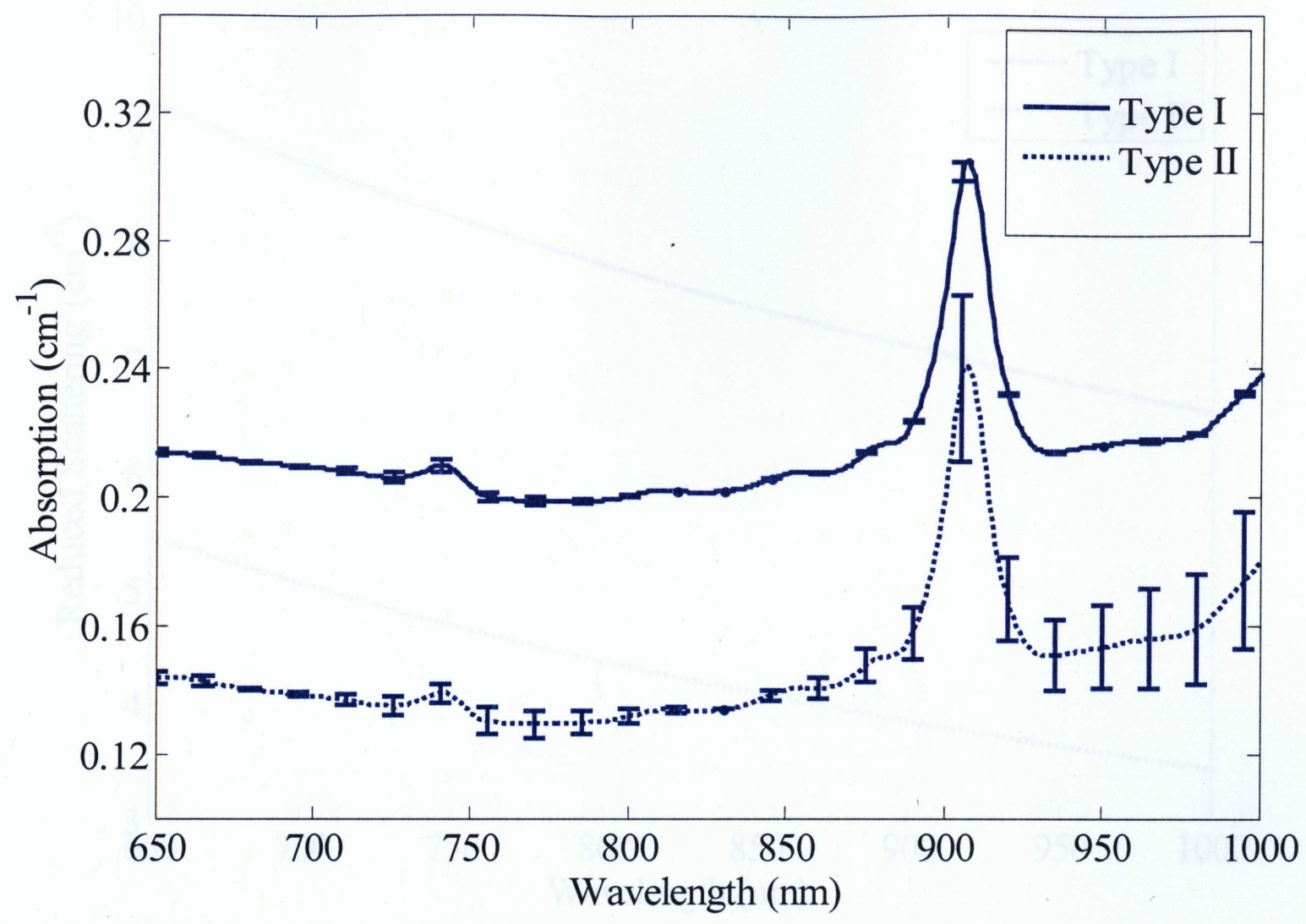

Figure 3. Absorption spectra of each homogeneous phantom. 


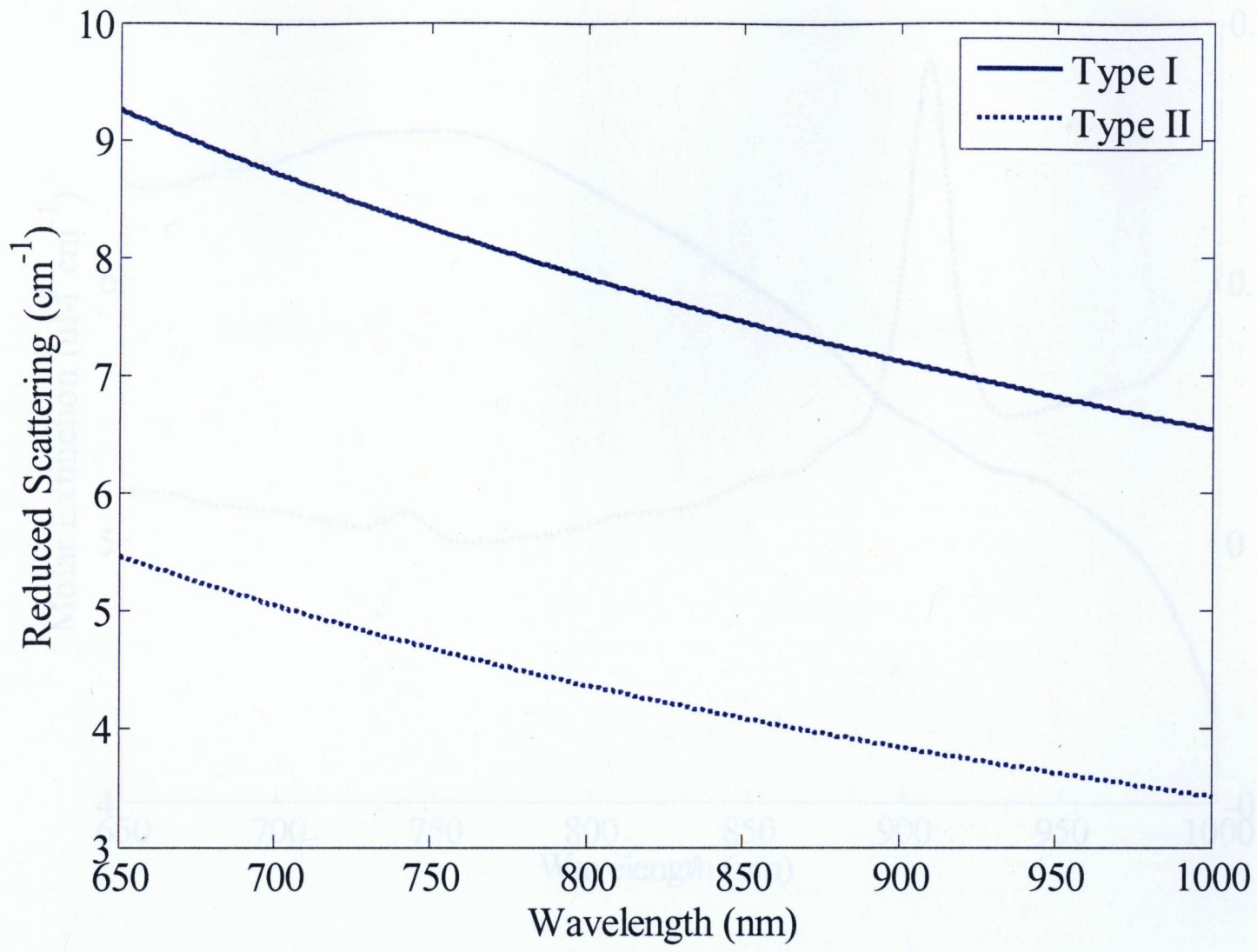

Figure 4. Reduced Scattering Coefficient Spectra of homogeneous phantoms. 


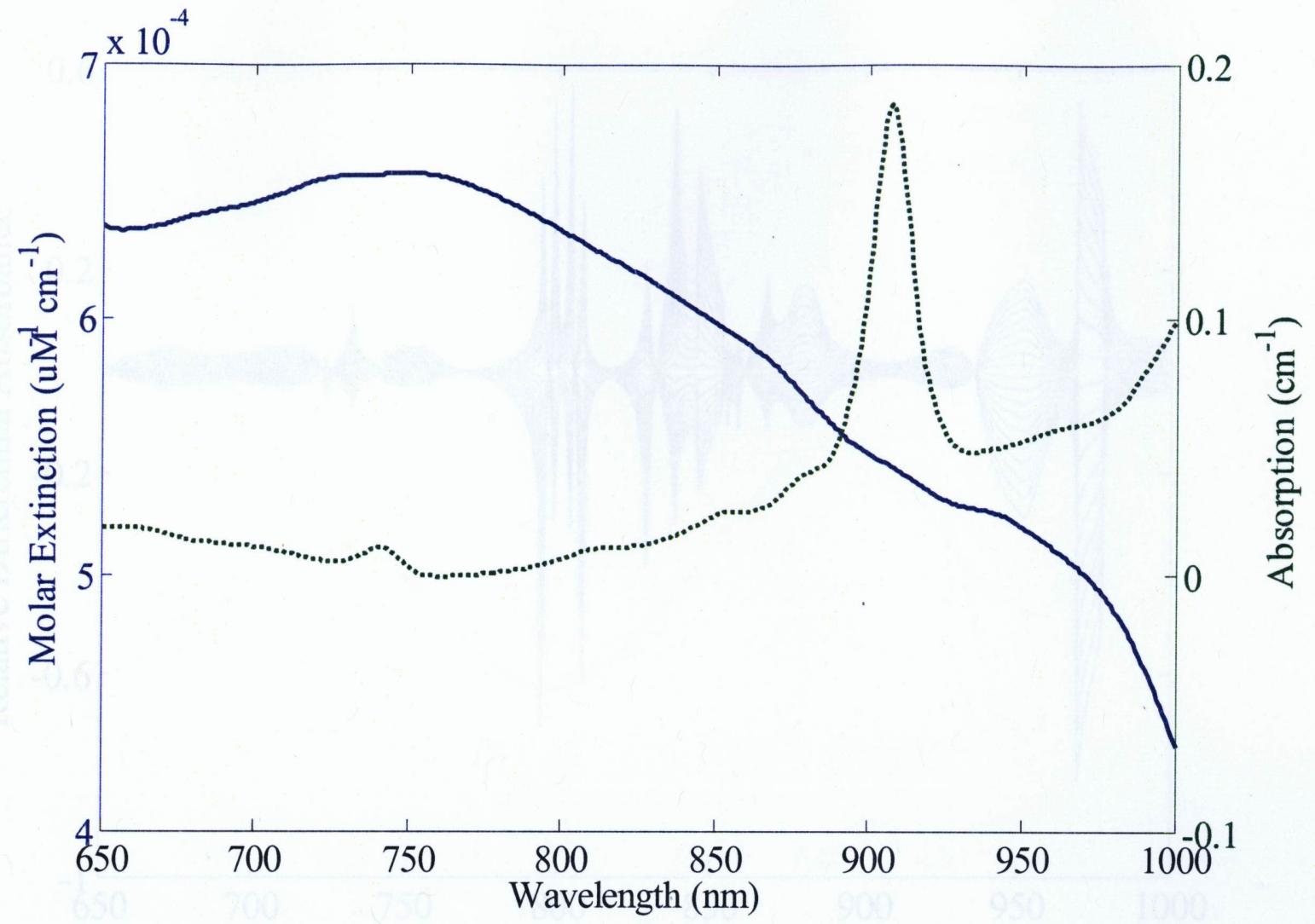

Figure 5. Silicone absorption spectrum and molar extinction of carbon black. 


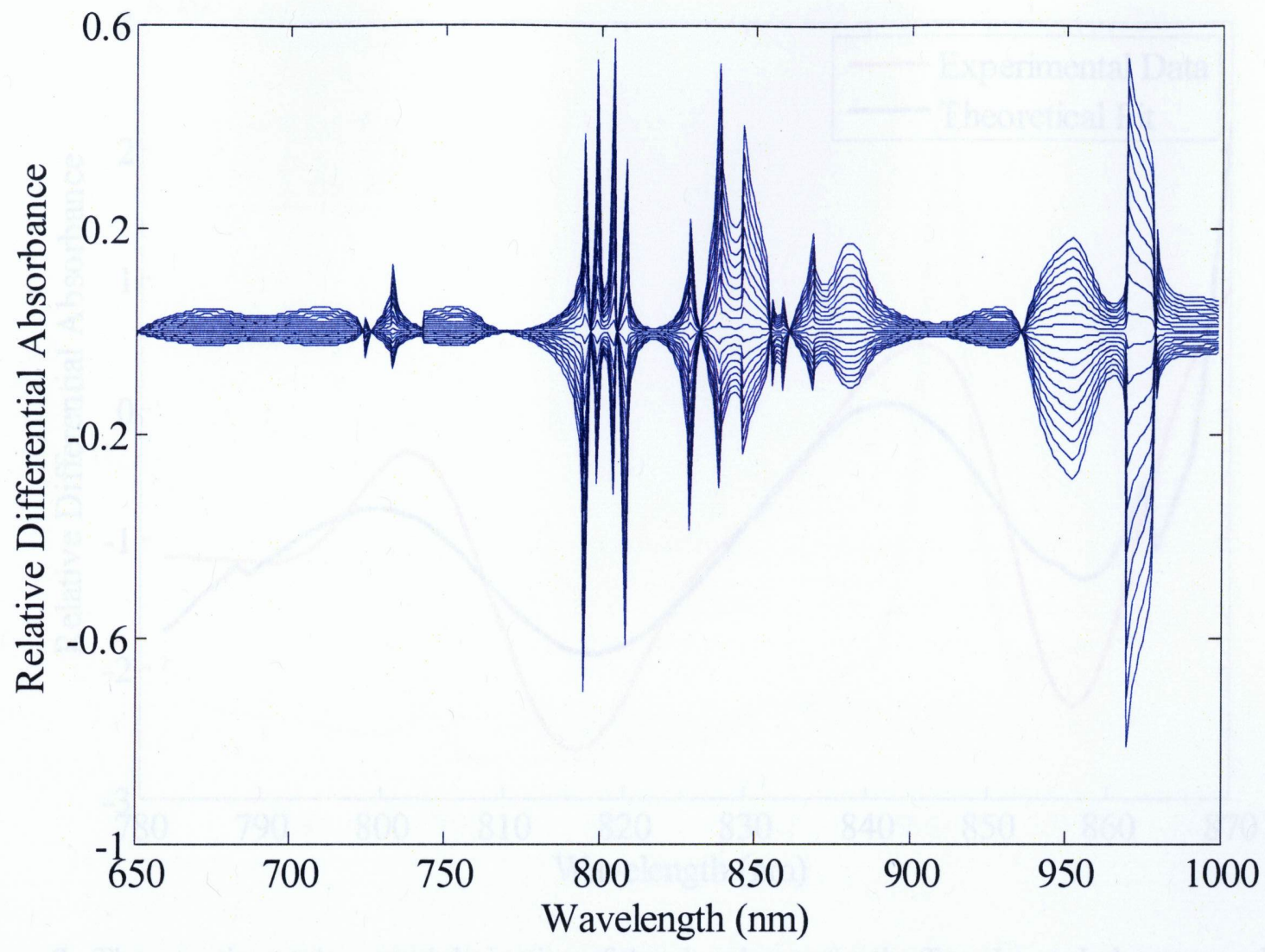

Figure 6. Theoretical differential absorbance sensitivity to relative changes in $\mu_{\mathrm{a}}$ of carbon black with constant scattering for two-layer phantom model. 


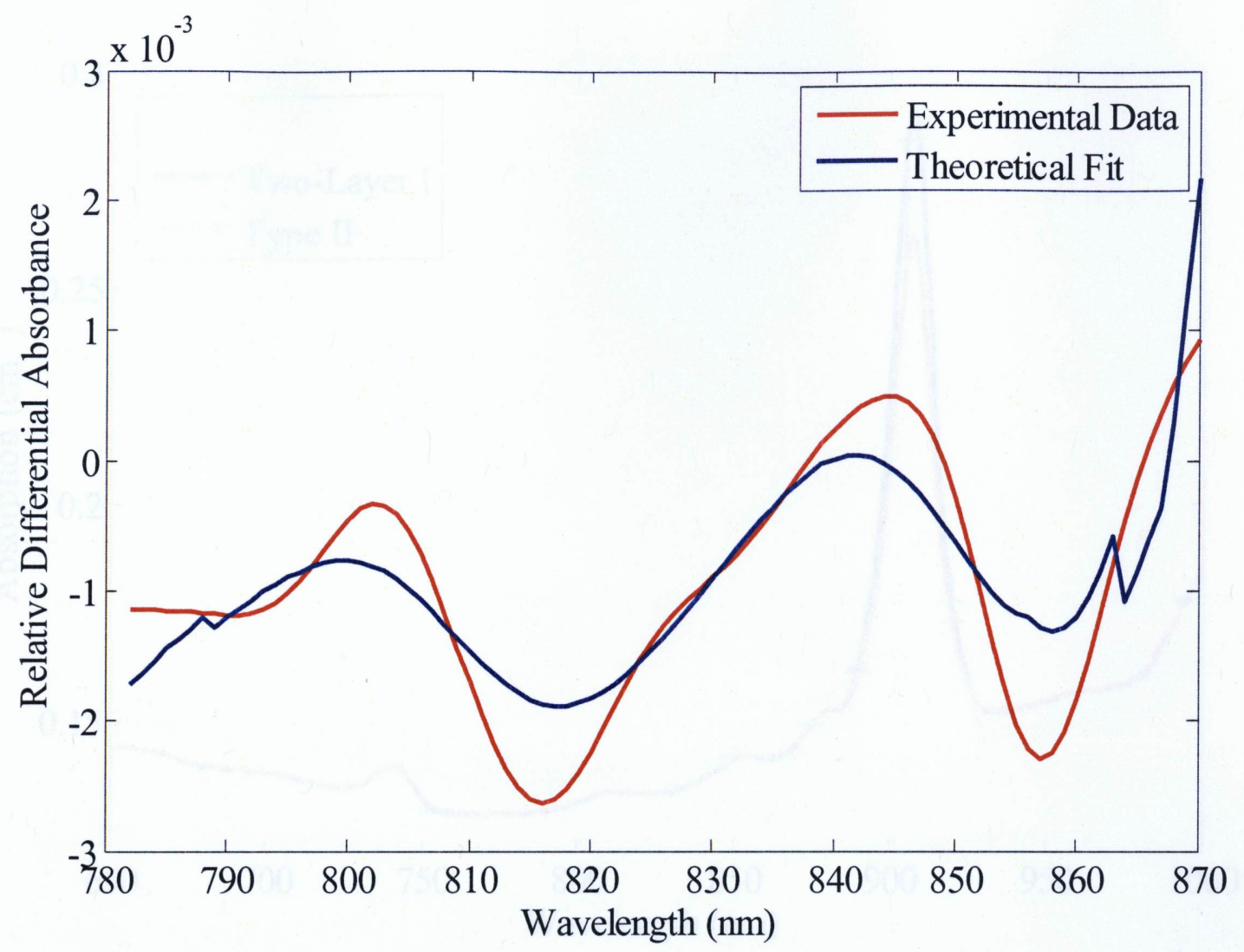

Figure 7. The experimental spectral derivative of the absorbance for the Two-Layer I phantom and the theoretical fit. 


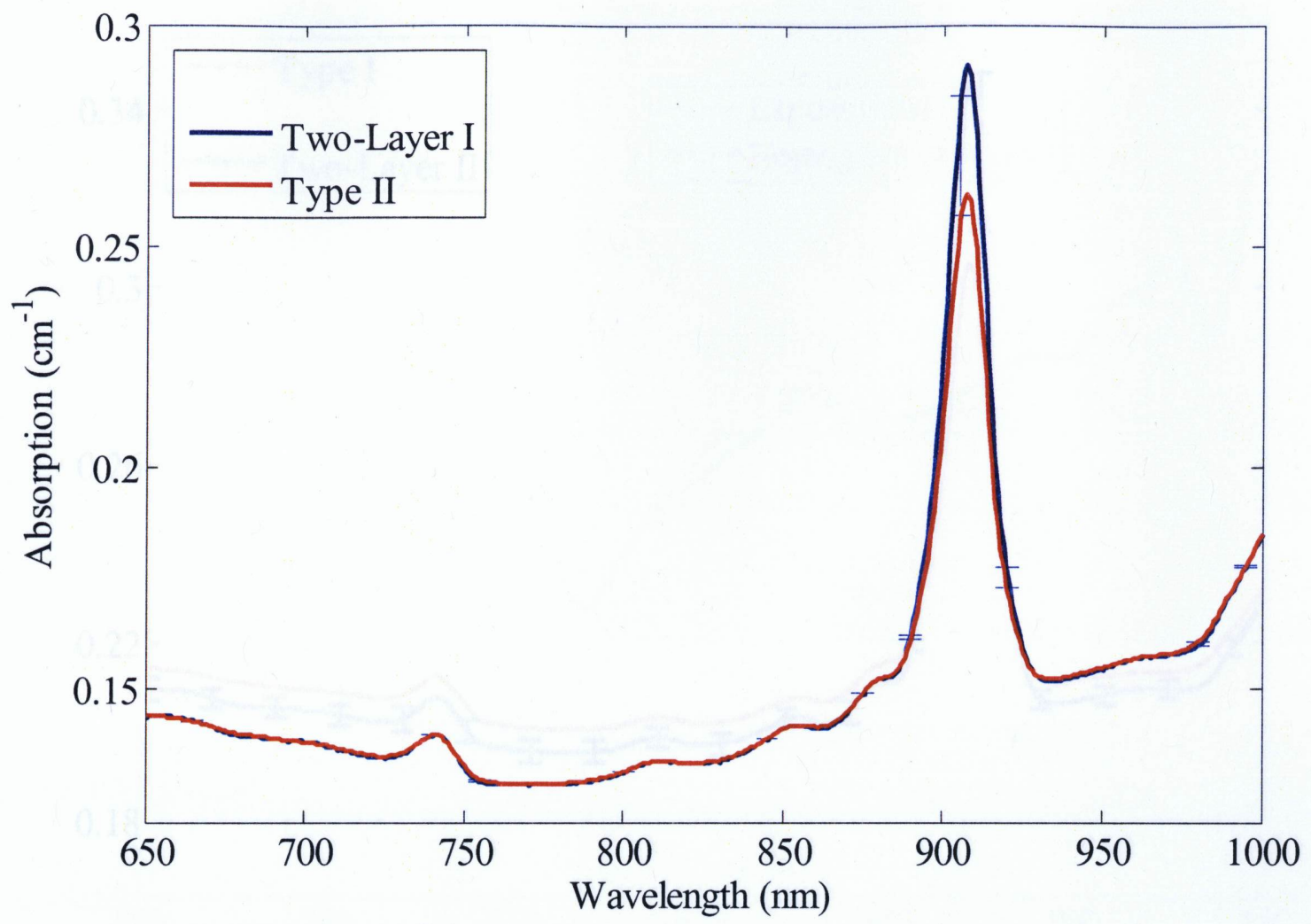

Figure 8. A comparison of the reconstructed absorption spectrum of the Type II bottom layer of the TwoLayer I phantom with the absorption spectrum obtained from measurements on the homogeneous Type II medium. 


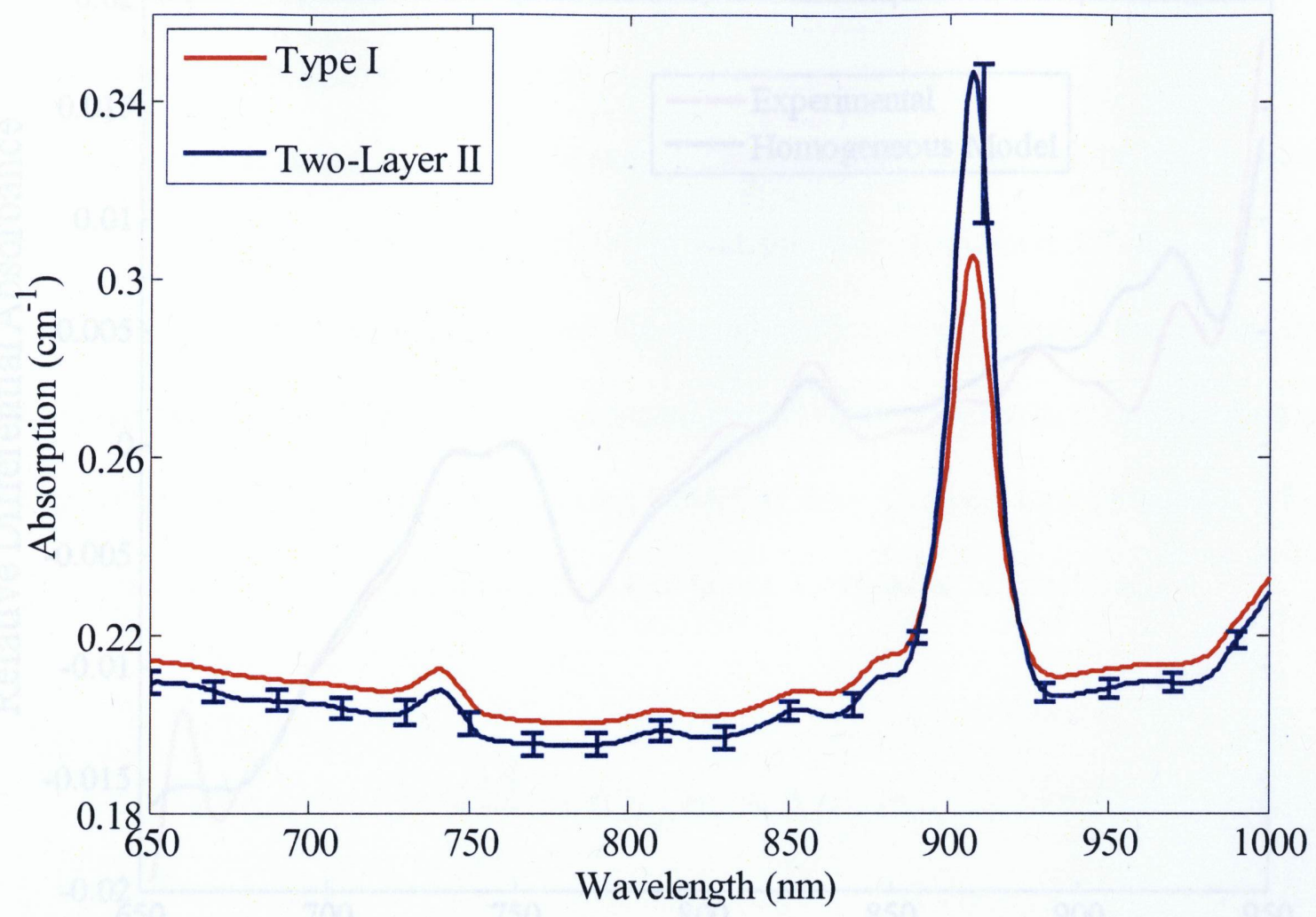

Figure 9. A comparison of the reconstructed absorption spectrum of the Type I bottom layer of the TwoLayer II phantom with the absorption spectrum obtained from measurements on the homogeneous Type I medium. 


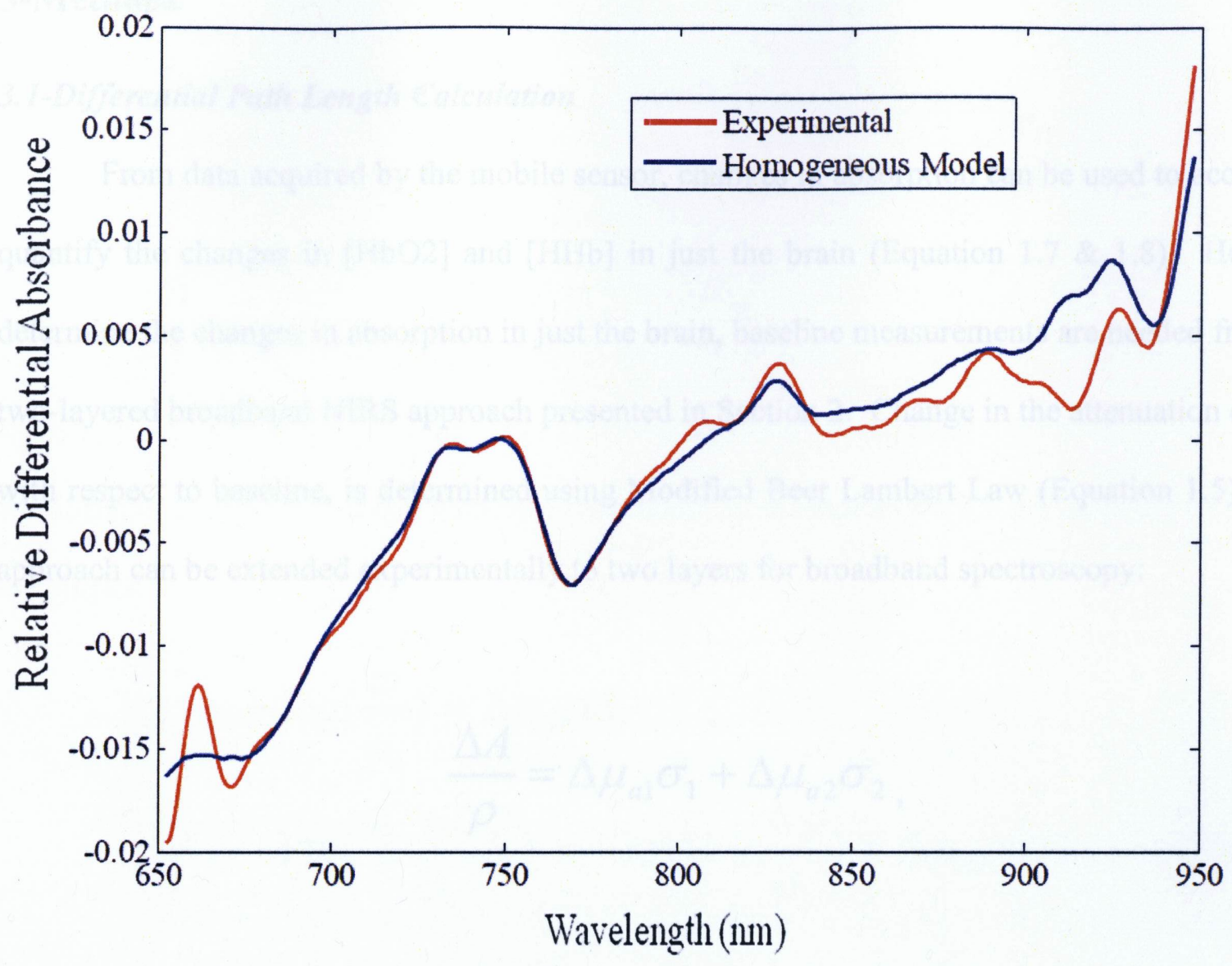

Figure 10. The experimental spectral derivative of the absorbance of human forehead at SD distance of 25 $\mathrm{mm}$ and the theoretical fit using the homogeneous model. 


\section{3-Methods}

\section{1-Differential Path Length Calculation}

From data acquired by the mobile sensor, changes in absorption can be used to accurately quantify the changes in $[\mathrm{HbO} 2]$ and $[\mathrm{HHb}]$ in just the brain (Equation $1.7 \& 1.8$ ). Hence to determine the changes in absorption in just the brain, baseline measurements are needed from the two-layered broadband NIRS approach presented in Section 2. Change in the attenuation of light with respect to baseline, is determined using Modified Beer Lambert Law (Equation 1.5). This approach can be extended experimentally to two layers for broadband spectroscopy:

$$
\frac{\Delta A}{\rho}=\Delta \mu_{a 1} \sigma_{1}+\Delta \mu_{a 2} \sigma_{2},
$$

where $\Delta \mu_{a 1}$ and $\Delta \mu_{a 2}$ represent the changes in absorption for the top and bottom layers respectively, $\sigma_{1}$ is the DPF for the top layer and $\sigma_{2}$ represents the DPF for the bottom layer. Using a probe with multiple light channels one could use Equation (3.1) to separate changes in the top (scalp and skull) and bottom (brain) layers. However, since the probe used in this research had only one fixed source-detector distance, it was assumed that the top layer does not change and that all hemodynamic changes occur just in the brain. Therefore assuming changes in absorbance only correspond to changes in $\Delta \mu_{a 2}, \sigma_{2}$ can be determined numerically using the baseline optical properties for the bottom layer measured in the previous section,. Fluence is dependent on the absorption coefficients of both top and bottom layers assuming scattering 
remains constant. If it is assumed that all physiological changes occur in the bottom layer, the corresponding change in fluence $\varphi$ is:

$$
\Delta \varphi=\frac{\partial \varphi}{\partial \mu_{a_{2}}} \Delta \mu_{a_{2}}
$$

Recalling equation 1.5 and using above Equation (3.2), an expression for the DPF can be derived as follows:

$$
\begin{aligned}
& -\log \left(\frac{\varphi}{\varphi_{o}}\right)=\Delta \mu_{a} \rho \sigma \\
& -\log \left(\frac{\varphi_{o}+\Delta \varphi}{\varphi_{o}}\right)=-\log \left(1+\frac{\Delta \varphi}{\varphi_{o}}\right)=\Delta \mu_{a} \rho \sigma
\end{aligned}
$$

Assuming a small relative change in $\varphi$, Equation (3.3) yields:

$$
-\frac{\Delta \varphi}{\varphi_{o}}=\Delta \mu_{a_{2}} \rho \sigma
$$

Replacing $\Delta \varphi$ with Equation (3.2) allows for an expression to be obtained for the DPF: 


$$
\sigma_{2}=\frac{-\partial \varphi / \partial \mu_{a_{2}}}{\rho \varphi_{o}}
$$

Where $\rho$ represents the source-detector (SD) distance and $\varphi_{\mathrm{o}}$ is the fluence at baseline conditions. Using the above equation, baseline DPF corresponding to the path length which reaches the brain $\left(\sigma_{2}\right)$ was calculated over the entire NIR spectrum for a SD distance of $25 \mathrm{~mm}$ (the distance of the SD of the mobile sensor).

\section{2- Smoking Measurements}

\subsection{1-System Overview}

A mobile near-infrared (NIR) sensor was used for acquiring changes in cerebral $\mathrm{HbO} 2$ and $\mathrm{HHb}$ concentrations during smoking. The portable sensor (Figure 3-1) was small in size and light in weight (about 50g), making the device easily wearable on the head. The device had four bi-wavelength (at 730 and $830 \mathrm{~nm}$ ) light emitting diode sources, and four light detectors. The distance between each source and detector was $25 \mathrm{~mm}$, which is considered optimal distance for light to reach the human brain for an average adult head [47]. Data acquired by the sensor was transmitted to a Personal Digital Assistant (PDA) via a Bluetooth channel and then retransmitted to a server via the GSM wireless network using the HTTP protocol. Loadable software to the PDA was developed which supports the above wireless data transfers [83]. 


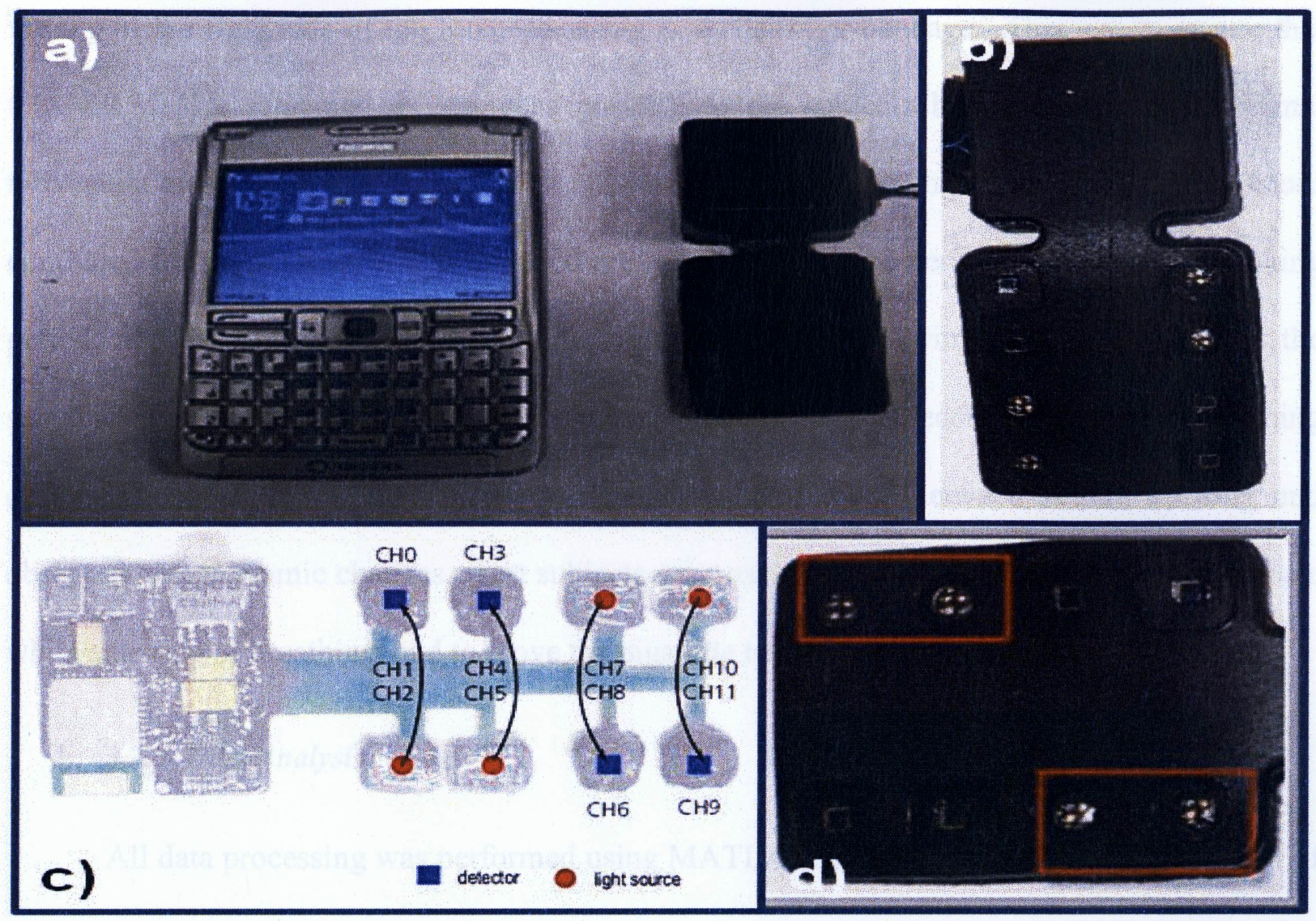

Figure 3-1- a) Mobile NIR sensor with portable phone b-d) Mobile sensor light emitters and detectors. The device had four bi-wavelength (at 730 and $830 \mathrm{~nm}$ ) light emitting diode sources, and four light detectors. Sensor courtesy of M. Wolf (Arquatis GmbH, Rieden, Switzerland).

\subsection{2- Human Subjects and Experimental Protocol}

A total of 20 measurements were preformed on 11 different adult subjects between the ages of 19 and 51. After informing each test subject of the nature of the study, each gave their consent. The protocol was approved by the Ryerson University Research Ethics Board (file number REB 2008-003). Each test subject reported that they have been smokers for at least one year and during the experiments they each used their own legally purchased brand of cigarettes. The human subject was then sat on a comfortable seating outdoors in a shelter covering to protect them from precipitations and direct sunlight at daylight. The NIR sensor was attached to 
the top of the right side of the forehead, using a sterile roller bandage. This was to ensure that the NIR device remained as stable as possible to the subjects head, allowing for minimal movement disturbance and to prevent signal detection of surrounding natural light. Each record continued for 15 minutes, which included a five-minute baseline period, a five-minute smoking period, and a five-minute recovery period without smoking. During the smoking period the subject was asked to inhale the smoke from the cigarette every 30 seconds. Such a regular timing of the procedure was used in order to determine correlations between smoke inhaling and observed hemodynamic changes. Test subjects were asked to smoke normally, not to over inhale while smoking or breathing, and to move the cigarette to their mouth very slowly.

\subsection{3- Data Analysis}

All data processing was performed using MATLAB 2007b (The MathWorksTM). Out of 20 data sets, 7 indicated the presence of artifacts due to motion or ambient light, hence were excluded from the analysis. Using the DPF ( $\sigma_{2}$ calculated in baseline measurements mentioned in Section 3.1) and the changes in absorbance acquired with the mobile sensor, changes in the absorption coefficient were calculated at the optical signals of $730 \mathrm{~nm}$ and $830 \mathrm{~nm}$ using the following relationship:

$$
\Delta \mu_{a_{\lambda}}=\frac{\Delta A_{\lambda}}{\rho \sigma_{\lambda}}=\frac{-\log \left(\frac{I_{\text {Final }}}{I_{\text {Initial }}}\right)_{\lambda}}{\rho \sigma_{\lambda}}
$$


Where $I_{\text {Final }}$ and $I_{\text {Initial }}$ are the measured intensities before and after the concentration changes measurements obtained from the mobile sensor and $\rho$ is the SD distance of the mobile sensor. Once $\Delta \mu_{a}$ was determined for the wavelengths of 730 and $830 \mathrm{~nm}$, the changes in oxy- and deoxy-hemoglobin concentrations were computed with exclusion of the surrounding layers of the brain, using Modified Lambert-Beer's law [18, 31, 32] expressed earlier in Section 1.2.2.

Comparison was made between hymodynamic changes in the human brain determined using the two layered modeling, and those computed assuming homogeneous modeling. 


\section{4 - Differential Path Length Factor and Mobile Sensor Results and Discussion}

\section{1- Differential Path Length Factor and Mobile Sensor Results}

This section discusses only DPF and mobile sensor results. Results and discussion on the two-layered approach and in vivo baseline measurements are found in Chapter 2.

The DPF for a SD distance of $25 \mathrm{~mm}$ was calculated as a function of wavelength (Figure 4-1) using Equation 3.5. The general trend was as wavelength increased, DPF decreased except for the longer wavelengths where a peak around $960 \mathrm{~nm}$ was present. This is due to the strong feature of water at these wavelengths.

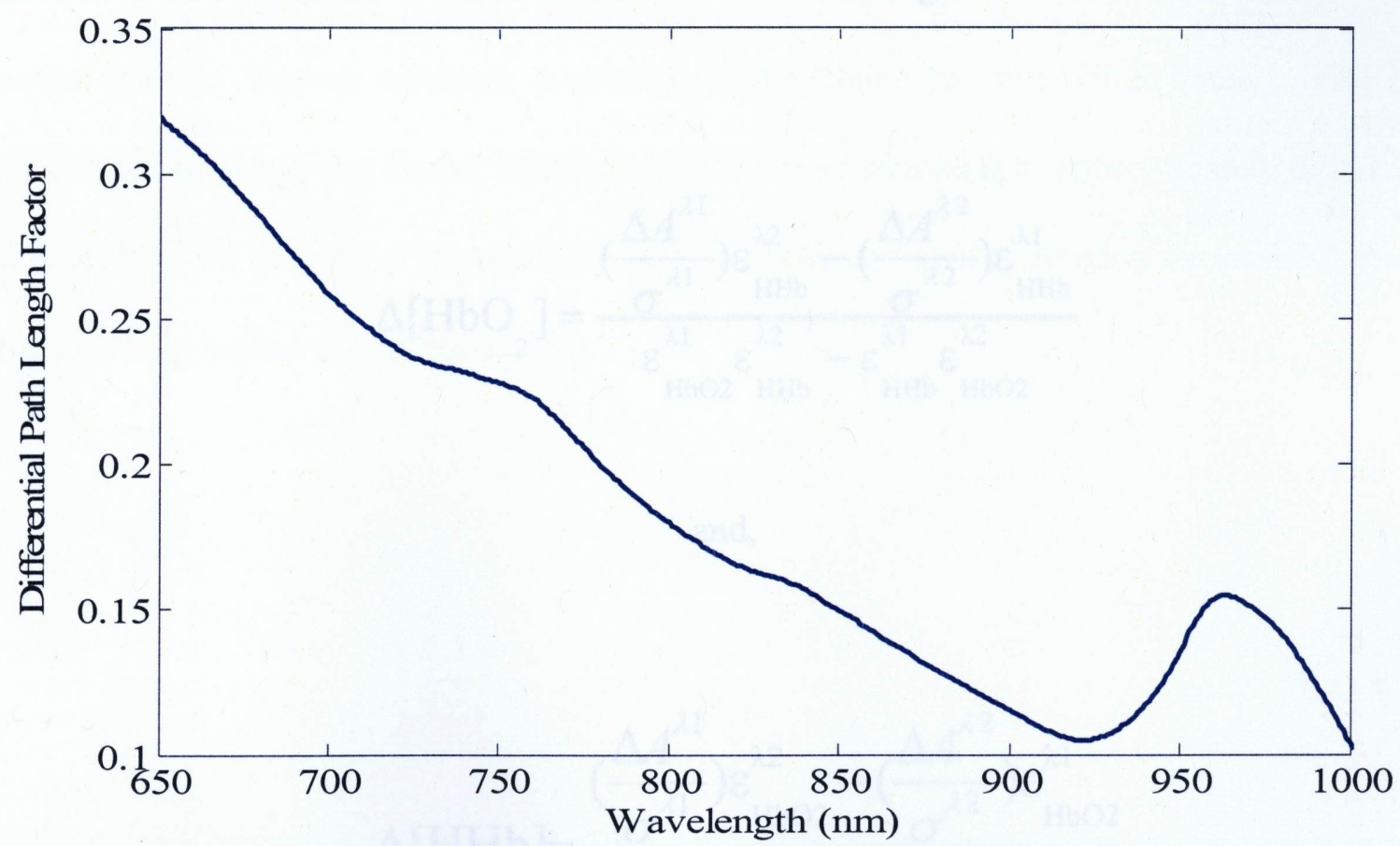

Figure 4-1- Differential path length factor for the second layer as a function of wavelength calculated using baseline optical properties of the bottom layer of a two-layered model of the human head, using broadband NIR spectroscopy at SD distance of $25 \mathrm{~mm}$. 
The DPF required for corresponding measurements obtained with the mobile sensor was found to be 0.227 and 0.1613 at the wavelength of $730 \mathrm{~nm}$ and $830 \mathrm{~nm}$ respectively.

Figure 4-2 presents the group averaged long-time hemodynamic changes assuming homogeneous medium during baseline, smoking and recovery periods. Figure 4-3 displays the averaged long-time hemodynamic changes from just the brain by applying the DPF's at $730 \mathrm{~nm}$ and $830 \mathrm{~nm}$ found in Figure 4-1 and Equations 1.7 and 1.8. To obtain the smoking data set assuming homogeneous medium (Figure 4-2), it was assumed that DPF remained the same for both wavelengths having a value of 6 [84], and Equations 1.7 and 1.8 was used. However, from Figure 4-1, it was found that DPF differs for the two wavelengths, hence Equation 1.7 and 1.8 is modified to account for the different DPF at the two wavelengths:

$$
\Delta\left[\mathrm{HbO}_{2}\right]=\frac{\left(\frac{\Delta A^{\lambda 1}}{\sigma^{\lambda 1}}\right) \varepsilon_{\mathrm{HHb}}^{\lambda 2}-\left(\frac{\Delta A^{\lambda 2}}{\sigma^{\lambda 2}}\right) \varepsilon_{\mathrm{HHb}}^{\lambda 1}}{\varepsilon_{\mathrm{HbO} 2}^{\lambda 1} \varepsilon_{\mathrm{HHb}}^{\lambda 2}-\varepsilon_{\mathrm{HHb}}^{\lambda 1} \varepsilon_{\mathrm{HbO} 2}^{\lambda 2}}
$$

and,

$$
\Delta[\mathrm{HHb}]=\frac{\left(\frac{\Delta A^{\lambda 1}}{\sigma^{\lambda 1}}\right) \varepsilon_{\mathrm{HbO} 2}^{\lambda 2}-\left(\frac{\Delta A^{\lambda 2}}{\sigma^{\lambda 2}}\right) \varepsilon_{\mathrm{HbO} 2}^{\lambda 1}}{\varepsilon_{\mathrm{HHb}}^{\lambda 1} \varepsilon_{\mathrm{HbO} 2}^{\lambda 2}-\varepsilon_{\mathrm{HHb}}^{\lambda 1} \varepsilon_{\mathrm{HbO} 2}^{\lambda 2}}
$$


Where $\Delta A^{\lambda l, 2}$ represents the change in absorbance and $\sigma^{\lambda l, 2}$ is the DPF for the brain at the wavelengths of $730 \mathrm{~nm}$ and $830 \mathrm{~nm}$ respectively. Equations 4.1 and 4.2 were used to calculate the changes in [HbO2] and [HHb] for heterogeneous tissue, accounting for the calculated DPF's of the brain at each specific wavelength. Comparing Figures 4-2 and 4-3, one can see the changes in hemoglobin concentrations were greatly underestimated when assuming homogeneous medium then compared to those found using DPF for the brain, by a factor of approximately 3 .

Qualitatively different types of the temporal behavior of cerebral hemodynamic changes during baseline, smoking and recovery periods were observed (Figure 4-3). [HbO2] and [HHb] increased for the first 150 seconds before leveling off and remaining stable for the rest of the baseline period. During smoking, a simultaneous increase in both $[\mathrm{HbO} 2]$ and $[\mathrm{HHb}]$ in the beginning of smoking was found, however [HbO2] reached a peak at approximately 520 seconds and decreased for the rest of the smoking period. Whereas, $[\mathrm{HHb}]$ steadily increased during the entire smoking period of the experiment. 


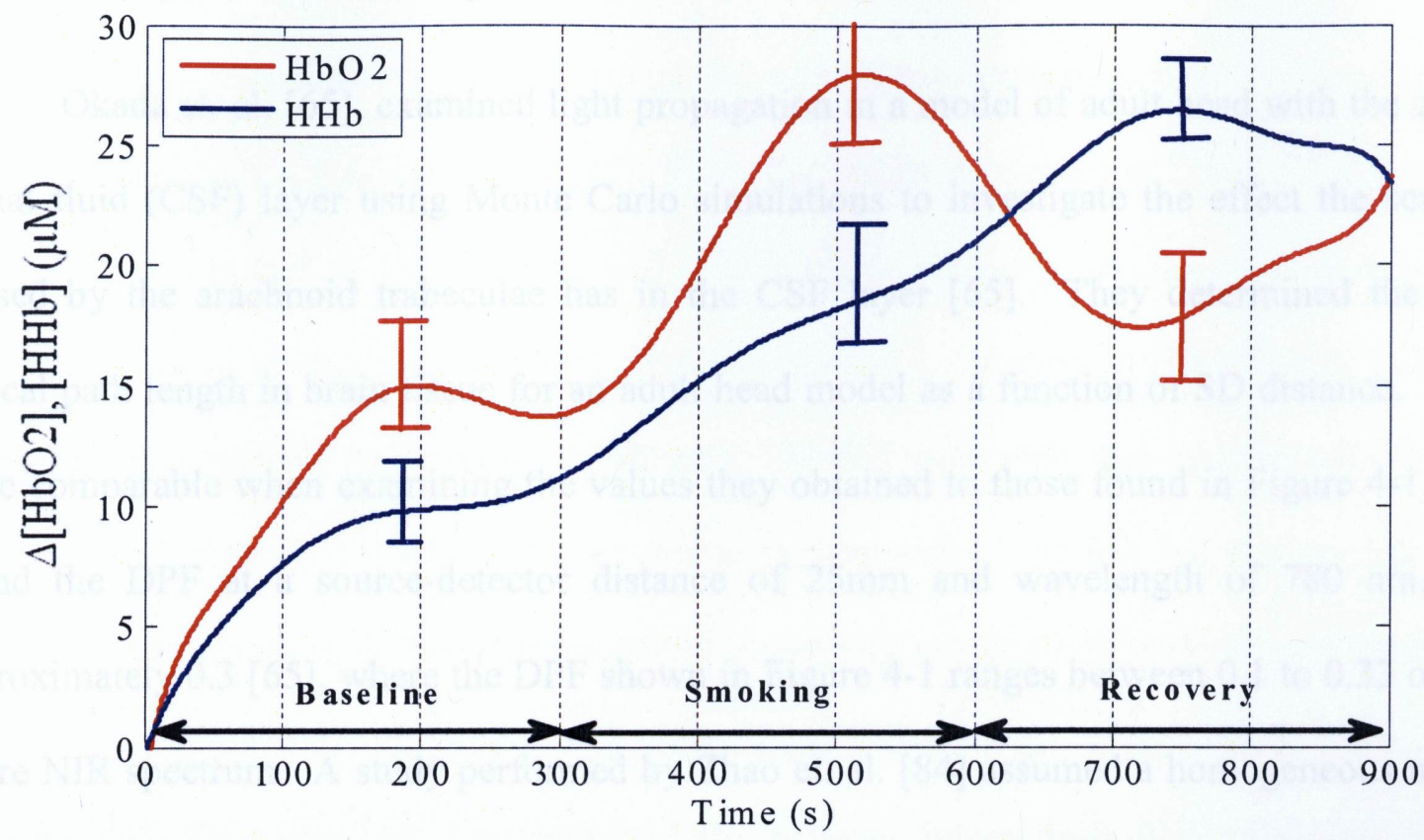

Figure 4-2- Group averaged long-time hemodynamic changes assuming homogeneous medium during baseline, smoking and recovery periods. Traces were averaged over four channels in 13 subjects. Error bars show the standard error.

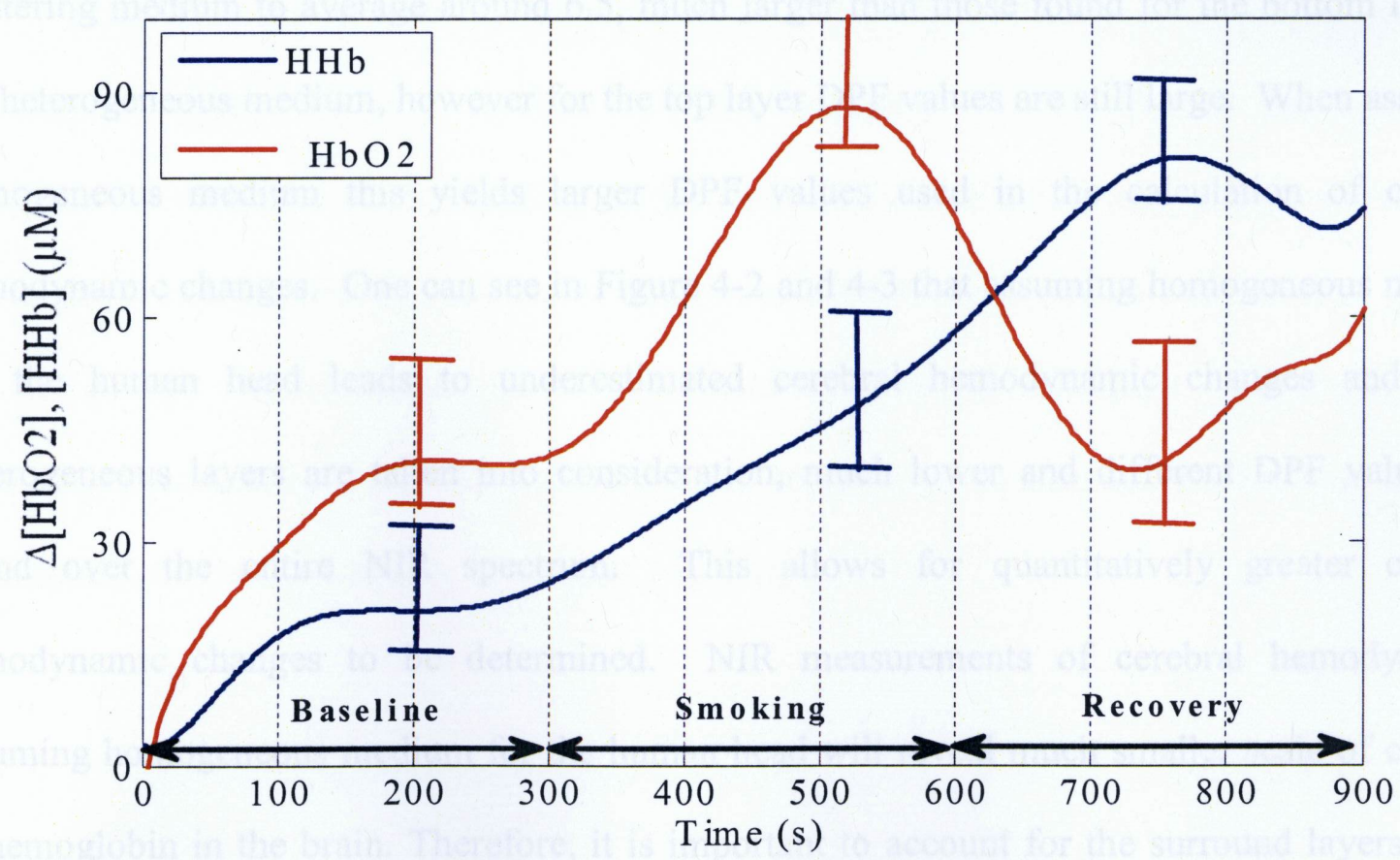

Figure 4-3- Group averaged long-time hemodynamic changes from just the brain by using the DPF's at 730 $\mathrm{nm}$ and $830 \mathrm{~nm}$ from Figure 4-1, during baseline, smoking and recovery periods. Traces were averaged over four channels in 13 subjects. Error bars show the standard error. 


\section{2- Differential Path Length Factor and Mobile Sensor Discussion}

Okada et. al. [65], examined light propagation in a model of adult head with the cerebral spinal fluid (CSF) layer using Monte Carlo simulations to investigate the effect the scattering caused by the arachnoid trabeculae has in the CSF layer [65]. They determined the partial optical path length in brain tissue for an adult head model as a function of SD distance. Values were comparable when examining the values they obtained to those found in Figure 4-1. They found the DPF at a source-detector distance of $25 \mathrm{~mm}$ and wavelength of $780 \mathrm{~nm}$, to be approximately 0.3 [65], where the DPF shown in Figure 4-1 ranges between 0.1 to 0.33 over the entire NIR spectrum. A study performed by Zhao et. al. [84] assumed a homogeneous medium and found DPF at the surface of the forehead using time-resolved NIRS at a SD of $30 \mathrm{~mm}$ and wavelengths of $759 \mathrm{~nm}, 700 \mathrm{~nm}$ and $834 \mathrm{~nm}$. They found DPF values for a homogeneous scattering medium to average around 6.5 , much larger than those found for the bottom layer of the heterogeneous medium, however for the top layer DPF values are still large. When assuming homogeneous medium this yields larger DPF values used in the calculation of cerebral hemodynamic changes. One can see in Figure 4-2 and 4-3 that assuming homogeneous medium for the human head leads to underestimated cerebral hemodynamic changes and when heterogeneous layers are taken into consideration, much lower and different DPF values are found over the entire NIR spectrum. This allows for quantitatively greater cerebral hemodynamic changes to be determined. NIR measurements of cerebral hemodynamics assuming homogeneous medium for the human head will reveal much smaller scale of changes of hemoglobin in the brain. Therefore, it is important to account for the surround layers of the brain. 
A combination of increases in [ $\mathrm{HbO} 2]$ and decreases in $[\mathrm{HHb}]$ is typical for changes observed during functional brain activations, and usually results from increases in cerebral blood flow, which causes a washout effect [85]. From the results presented in Figures 4-2 and 4-3, it seems that at the end of the smoking and beginning of recovery periods cerebral blood flow sharply decreased at some point during smoking causing a decrease in [HbO2] and increase in $[\mathrm{HHb}]$ concentrations. At baseline conditions, $[\mathrm{HbO} 2]$ increased and leveled off at greater concentrations then $[\mathrm{HHb}]$. This may be due to factors such as the test subjects not being fully relaxed at the beginning of the experiment and eventually calming half way through the baseline period. During the recovery period, both $[\mathrm{HbO} 2]$ and $[\mathrm{HHb}]$ began to stabilize at the end of the time period. This suggests that smoking effects lasted longer than 5 minutes and a longer recovery period was required for $[\mathrm{HbO} 2]$ and $[\mathrm{HHb}]$ to return back to the baseline.

A recent positron emission tomography (PET) study by Brody et al. [82] showed that smoking a regular cigarette resulted in $88 \%$ occupancy of the human brain nicotinic acetylcholine receptors. Since binding of the nicotine molecules to these receptors resulted in excitation in neurons and the release of neurotransmitters, hence one can expect that smoking can also cause a continuous increase in oxygenation and cerebral blood flow. The averaged data showed increases in both $[\mathrm{HbO} 2]$ and $[\mathrm{HHb}]$ and hence in blood volume. An increase in blood volume can indicate an increase in cerebral blood flow. 


\section{5 - Conclusions and Future Work}

\section{1- Conclusions}

Studies of cerebral hemodynamics require fast, non-invasive measuring techniques. NIRS provides a versatile method for non-invasive studies of the circulation system of the human brain, but it requires elimination of signal contribution from the skull. This work supports the previously stated hypothesis such that, through the use of broadband NIRS the optical properties in a two-layered model of the human head are determined. It also revealed the significant underestimation in the quantification of changes in [HbO2] and [HHb] when assuming a homogeneous medium for cerebral measurements.

A broadband spectral approach to measure absolute optical properties of heterogeneous tissue with layered architecture has been presented. The approach is based on the diffusion approximation solution for the semi-infinite homogeneous two-layer medium and a least squares fit of the first spectral derivative of the absorbance. The optical properties of the bottom layers in two-layer phantoms with $15 \mathrm{~mm}$ thick top layers were successfully measured. Encouraging preliminary in-vivo results provide reasonable cerebral hemoglobin concentrations and oxygenation in healthy adult humans. Past research has already applied multi-layer approaches in biomedical NIRS $[9,60,63]$. In particular, Alexandrakis et al. investigated the possibility of determining the optical properties of a two-layered medium using spatially resolved FD reflectance [86]. They used two phantom models: the first consisted of skin overlying muscle and the other of skin overlying fat, each having varying top-layer thicknesses between 1-4mm. It was assumed the upper layer thickness was unknown, and they determined the optical parameters in both layers. The Monte Carlo simulation model and the two-layer diffusion solution were 
fitted to experimental frequency-domain reflectance measurements of their models, and both could determine the optical properties of the lower layers [86]. In their research, the use of FD measurements with light from a $677 \mathrm{~nm}$ laser diode, intensity modulated at $100 \mathrm{~Hz}$, only allowed for the researchers to examine data at a discrete wavelength. In addition, the use of Monte Carlo simulations to determine the optical properties of two layers required a high amount of computational time [87]. However, a very first study combining multi-layer diffusion theory with the broadband $\mathrm{CW}$ type of measurements is presented in this thesis.

In the broadband approach, the first spectral derivative of the absorbance was used, unlike previous broadband approaches [87] which use the second spectral derivatives, and fit to experimental data using tabulated chromophore spectra and concentrations as fitting parameters. In baseline measurements, it was shown that with the broadband two-layered approach, it is feasible to recover the optical properties of the bottom layer representing the brain, covered with a thick top layer $(15 \mathrm{~mm})$ which corresponds to the thickness of the surrounding layers of an average adult human head [62]. The phantom studies used for testing the method have supported the feasibility of the broadband approach, even though the extinction spectrum of carbon black was less distinct in the NIR spectrum than natural tissue chromophores. However, tissue chromophores, such as water, have much more pronounced NIR spectral features, hence it is expected that the broadband approach will be more practical in biomedical NIRS such as the detection of cerebral hypoxia in adults, children and neonates.

Through an extended approach of Modified Lambert Beer Law, the DPF over the entire NIR spectrum was determined for the bottom layer. Values obtained were consistent with those found in past research [65]. Determination of the DPF for the bottom layer at wavelengths of 
$730 \mathrm{~nm}$ and $830 \mathrm{~nm}$ allowed for the accurate quantification of changes in [HbO2] and [HHb] obtained with the use of a mobile wireless NIR sensor. Analyzed measurements were those obtained on test subjects while smoking in order to determine the effect naturally administered smoke has on cerebral hemodynamics. Comparison made between the group averaged long-time hemodynamic changes by applying DPF for the brain and assuming homogeneous medium for the adult human head, displayed a great underestimation in concentration changes in the two chromophores when assuming a homogeneous medium. Hence it is important to consider the surrounding layers of the human head when performing measurements related to cerebral hemodynamic changes.

In regards to the general trends found in $[\mathrm{HbO} 2]$ and $[\mathrm{HHb}]$ changes and smoking, the results suggested that smoking can cause fast and random spontaneous changes in cerebral blood flow. The results also indicated that portable wireless NIRS devices can be very useful in measuring hemodynamic parameters in natural environments. In particular, the mobile technique presented, is capable of measuring changes in hemoglobin concentrations in the brain while the test subject is smoking. This allowed for measured effects of naturally administered smoke, which is a limitation to other imaging modalities. Developing a mobile NIR sensor technology can provide a significant contribution to clinical practices, allowing for physiological measurements to be performed on human subjects in their natural environment. 


\section{2- Future Work}

The following summarizes some key areas that need further investigation in determining the optical properties of the brain with removal of the effects from surrounding tissue layers using broadband NIRS, and for determining the effect naturally administered smoke has on cerebral hemodynamics:

1) Examine the contributions of additional layers in modeling the human head to gain more accurate measurements of hemodynamic changes in the brain. It has been shown in past research that specific layers distort the behaviour of NIR light, specifically the scalp fat that can cause a great effect in the amount of NIR light that reaches the brain and may be more beneficial to model it as a separate layer. As well, increasing the number of layers in the model for the human head will allow for a greater accuracy in the obtained optical properties of the brain.

2) Certain issues arose from measurements taken on heterogeneous tissue (described in baseline measurements Section 2), such as a large decrease in the scattering at longer NIR wavelengths. A better understanding of this result needs to be gained.

3) Measurements taken on heterogeneous tissue need to be examined for different areas of the adult head, not just limited to the forehead. This will allow for the examination of optical properties of the brain at different locations and whether a predominant difference is present. However, influential factors need to be overcome to obtain measurements on areas of the head, such as hair. 
4) Examine long term physiological effects of smoking on the brain using broadband NIRS, to examine cerebral hemodynamic changes in the brain to different stimuli with an inexpensive, non-invasive alternative biomedical approach.

5) Further study is needed into determining whether certain human parameters cause a difference in the data measurements, such as gender, age and the number of years the individual has been a smoker. This will require a larger number of measurements performed on test subjects.

6) Greater analysis is needed to determine whether [ $\mathrm{HbO} 2]$ and [ $\mathrm{HHb}]$ changes caused by smoking are localized to certain areas of the brain and their significance, for the cerebral health of the smoker. Determining the effects of smoking on cerebral hemodynamics will help advance research studies on nicotine and its adverse health effects 


\section{6 - Appendix}

This appendix contains the Matlab source code for the two-layered spectral analysis employed in this work.

\section{1) Determination of absorption spectra of phantoms}

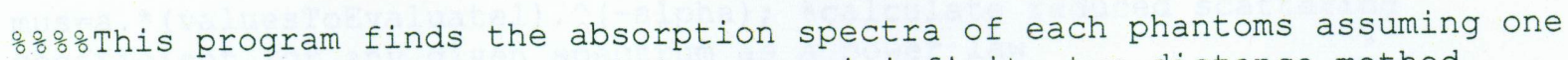
\%응으omogenous medium and applying a semi-infinite two-distance method

\%이tten by:

\%Olivia Pucci and Dr. Vladislav Toronov (August 2008)

\% Calculate kappa from the known optical property points

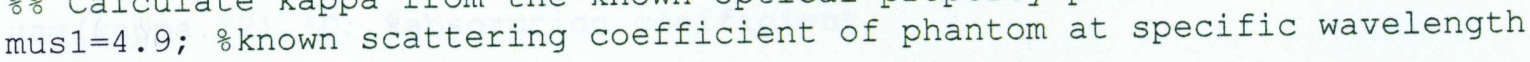
(690 nm) in $\mathrm{cm}$ mus $2=4.2$; $\%$ known scattering coefficient of phantom at specific wavelength $(830 \mathrm{~nm})$ in $\mathrm{cm}$

$\mathrm{k} 2=\operatorname{sqrt}(3 * \operatorname{mus} 1 * 0.139) ; \%$ Known kappa at $690 \mathrm{~nm}$

for $\mathrm{N}=40:-1: 1$;

k2=fzero $(a(K)$ Bottoml $(K, N), k 2)$; calls function Bottom1 to calculate kappa at the indicated wavelength position kappa3 $(\mathrm{N})=\mathrm{k} 2$;

end

$\mathrm{k} 3=\operatorname{sqrt}(3 *$ mus $1 * .139) ; \quad \%$ Known kappa at $690 \mathrm{~nm}$

for $N=41: 1: 180$;

$\mathrm{k} 3=\mathrm{fzero}(\mathrm{Q}(\mathrm{K}) \operatorname{Bottom} 1(\mathrm{~K}, \mathrm{~N}), \mathrm{k} 3)$;

kappa $4(\mathrm{~N})=\mathrm{k} 3$;

end

$\mathrm{k} 4=\operatorname{sqrt}(3 *$ mus $2 * 0.134) ; \%$ Known kappa at $830 \mathrm{~nm}$

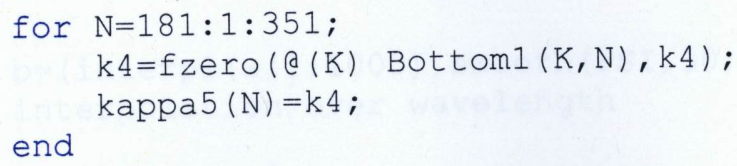

end

\% kappa $=[\operatorname{kappa} 3(:, 1: 40) \operatorname{sqrt}(3 * \operatorname{mus} 1 * 0.139) \quad \operatorname{kappa} 4(:, 42: 180) \operatorname{sqrt}(3 * \operatorname{mus} 2 * 0.134)$ kappa5 (:, 182:351) ] ';

kappa $=[\operatorname{kappa3}(:, 1: 40) \operatorname{kappa} 4(:, 41: 180) \operatorname{kappa}(:, 181: 351)]$; \%Kappa Matrix 
valuestoEvaluate $1=[650: 1: 1000]$ '; $\frac{\circ}{0}$ Wavelength matrix

음 Calculate Reduced Scattering Coefficient as a Power Law

lambdal=(690); 弚irst known wavelength of block for corresponding scattering coefficient

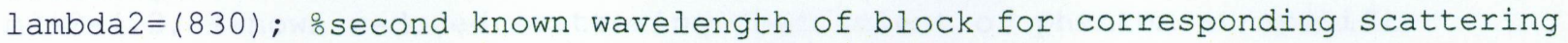
coefficient

alpha $=-((\log (\operatorname{mus} 2 / \operatorname{mus} 1)) /(\log (\operatorname{lambda} 2 / \operatorname{lambda} 1)))$; $c a l c u l a t e$ alpha a=mus2/(lambda2^(-alpha)); \%calculate coefficient

mus=a.* (valuesToEvaluatel).^(-alpha); calculate reduced scattering coefficient for any given spectrum as a power law

응 Calculate Absorption Coefficient Spectra's

$D=\left(1 . /\left(3 .{ }^{*}\right.\right.$ mus $\left.)\right)$; odiffusion constant for any given reduced scattering coefficient

ua $=\left(\right.$ kappa.^2) $\cdot{ }^{\star} D ;$ oabsorption coefficient

figure

plot(valuesToEvaluate1,ua) oplot absorption coefficient spectra over the wavelength range of 650-1000nm

응으은

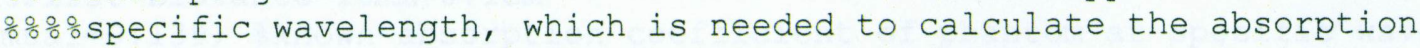

$\therefore \circ \frac{0}{0} \frac{\circ}{\circ}$ spectra of the specific phantom of interest

o은itten by:

o일ia Pucci and Dr. Vladislav Toronov (August 2008)

function $y=\operatorname{Bottom} 2(K, N)$

\% Curve Smoothing and determining wavelength interval and PSI for specific values

load $\mathrm{w}$ \%load wavelength

load db20 \%load absorbance measurement

PSI $=\mathrm{db} 20(1: 1000) .{ }^{*} \log (10) ; \quad$ convert data to natural logarithm

$\mathrm{b}=($ interpl $(\mathrm{w}(1: 1000)$, smooth $(P S I, 30), 650: 1: 1000)) ' ; \quad \%$ smooth and data

interpolation over wavelength

valuesToEvaluate $1=[650: 1: 1000] '$; owavelengths in $\mathrm{nm}$

Clear PSI

$\mathrm{PSI}=\mathrm{b}$; 
$\%$ Calculates wavelength dependence of the reduced scattering coefficient as a power law

mus $1=4.9$; $\frac{\circ}{3}$ known reduced scattering coefficient of phantom at specific wavelength $(690 \mathrm{~nm})$ in $\mathrm{cm}$ mus $2=4.2$; $\frac{\circ}{3}$ known reduced scattering coefficient of phantom at specific wavelength $(830 \mathrm{~nm})$ in $\mathrm{cm}$ lambdal $=(690)$; $\frac{0}{\text { known }}$ wavelength of block for corresponding reduced scattering coefficient

lambda2 $=(830)$; oknown wavelength of block for corresponding reduced scattering coefficient

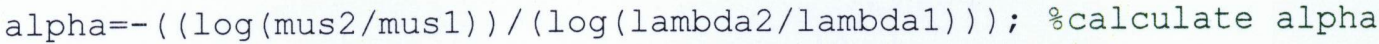
a=mus1/(lambda1^(-alpha)); ocalculate coefficient constant

mus=a.*(valuesToEvaluate 1$) \cdot \wedge(-a l p h a) ; ~ \% c a l c u l a t e ~ r e d u c e d ~ s c a t t e r i n g$ coefficient for any given spectrum

\% Plot reduced scattering coefficient over wavelength range of $650 \mathrm{~nm}$ to $1000 \mathrm{~nm}$ figure

hold on

plot (valuesToEvaluate1, mus)

title('Specific scattering coefficient in relation to wavelength')

hold off

응 Solve for log constant with known absorption coefficient points of phantom at two different wavelengths

을 Dirst Distance $10 \mathrm{~mm}=0.1 \mathrm{~cm}$ mual=0.139; $\%$ known absorption coefficient of phantom at specific wavelength of $690 \mathrm{~nm}$ in $\mathrm{cm}$ mua2=0.134; $\frac{\circ}{\mathrm{k} n o w n}$ absorption coefficient of phantom at specific wavelength of $830 \mathrm{~nm}$ in $\mathrm{cm}$

응 Calculate parameters needed to determine reflectance:

$\mathrm{D} 1=1 /(3 * \operatorname{mus} 1)$;

$\mathrm{D} 2=1 /(3 *$ mus 2$)$;

$\mathrm{K} 1=\operatorname{sqrt}((\operatorname{mua1}) / \mathrm{D} 1)$;

$\mathrm{K} 2=\operatorname{sqrt}((\operatorname{mua} 2) / \mathrm{D} 2)$;

$\mathrm{zb} 1=2 * \mathrm{D} 1$;

$z 01=3 * \mathrm{D} 1$;

$\mathrm{zb} 2=2 * \mathrm{D} 2$;

$\mathrm{z} 02=3 * \mathrm{D} 2$;

$\mathrm{x} 1=1.0 ; \%$ first distance in $\mathrm{cm}$

\%Begin: Reflectance in homogeneous medium for first S/D distance

o

$\mathrm{x} 11=\operatorname{sqrt}\left(\mathrm{x} 1 \cdot \wedge 2+\mathrm{z} 01^{\wedge} 2\right)$;

$x 2-1=\operatorname{sqrt}\left(x 1 \cdot{ }^{\wedge} 2+(z 01+2 * z b 1) \wedge 2\right) ;$

Fplus_1 $1=\exp \left(-\mathrm{K} 1 * \mathrm{x} 1 \_1\right) \cdot{ }^{*}\left(1+2 * \mathrm{D} 1 * \mathrm{z} 01 *\left(1+\mathrm{K} 1 * \mathrm{x} 11_{1}\right) \cdot / \mathrm{x} 1{ }_{1} 1 \cdot{ }^{\wedge} 2\right) \cdot / \mathrm{x} 1 \_1$;

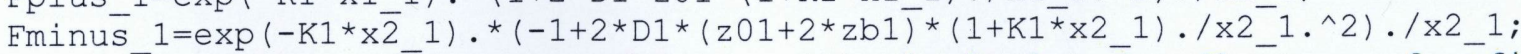
psi0_1= $\left(1 /\left(16 * \mathrm{pi}{ }^{*} \mathrm{D} 1\right){ }^{*}(\right.$ Eplus_1+Eminus_1)); $\%$ calculate $\bar{d}$ reflectance for first known absorption at $10 \mathrm{~mm}$ distance 
$x 121=\operatorname{sqrt}\left(x 1 \cdot \wedge 2+z 02^{\wedge} 2\right) ;$

$x 2-21=\operatorname{sqrt}(x 1 \cdot \wedge 2+(z 02+2 * z b 2) \wedge 2)$;

Fplus_21 $=\exp \left(-\mathrm{K} 2 * \mathrm{x} 1 \_21\right) \cdot{ }^{*}\left(1+2 * \mathrm{D} 2 * \mathrm{z} 02 *\left(1+\mathrm{K} 2 * \mathrm{x} 1 \_21\right) \cdot / \mathrm{x} 1{ }^{2} 21 \cdot{ }^{\wedge} 2\right) \cdot / \mathrm{x} 1{ }^{2} 21$;

Fminus_21=exp $(-\mathrm{K} 2 * x \overline{2}-21) \cdot *(-$

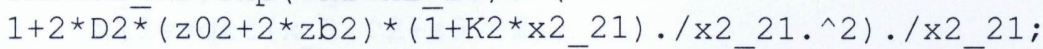

psi0_21=1/(16*pi*D2).*(Fplus_21+Fmiñus_21); ocallculated reflectance for

second known absorption at $2 \overline{5} \mathrm{~mm}$ distance

psi_1= (PSI(41)); omeasured reflectance for first known absorption at both distances

psi_2=(PSI(181)); omeasured reflectance for second known absorption at both distance

$\mathrm{x} 2=2$; $\frac{0}{8}$ Second distance in $\mathrm{cm}$

$\%$ Begin: Reflectance in homogeneous medium for second S/D distance

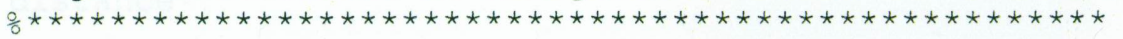

$x 112=\operatorname{sqrt}\left(x 2 \cdot{ }^{\wedge} 2+z 01 \wedge 2\right) ;$

$\mathrm{x} 2{ }^{-} 12=\operatorname{sqrt}\left(\mathrm{x} 2 \cdot{ }^{\wedge} 2+(\mathrm{z} 01+2 * \mathrm{zb} 1)^{\wedge} 2\right) ;$

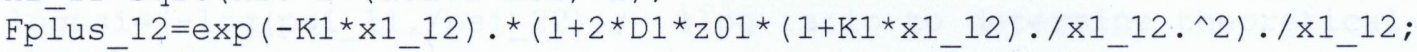

Fminus $12=\exp (-\mathrm{K} 1 * x \overline{2}-12) \cdot *(-$

$\left.1+2 * D 1{ }^{\star}(z 01+2 * z b 1) *\left(\overline{1}+K 1 * x 2 \_12\right) \cdot / x 2 \_12 \cdot \wedge 2\right) \cdot / x 2 \_12 ;$

psi0_12=(1/(16*pi*D1) **(Fpluss_12+Fminus_12)); $\overline{0}$ calculated reflectance for

first known absorption at distance of $2 \overline{0 m m}$

$\mathrm{x} 122=\operatorname{sqrt}\left(\mathrm{x} 2 \cdot \cdot^{\wedge} 2+\mathrm{z} 02^{\wedge} 2\right) ;$

$x 2-22=\operatorname{sqrt}(x 2 \cdot \wedge 2+(z 02+2 * z b 2) \wedge 2) ;$

Fplus $22=\exp \left(-\mathrm{K} 2 * \mathrm{x} 1 \_22\right) \cdot{ }^{*}\left(1+2 * \mathrm{D} 2 * \mathrm{z} 02 *\left(1+\mathrm{K} 2 * \mathrm{x} 1 \_22\right) \cdot / \mathrm{x} 1 \_22 \cdot{ }^{\wedge} 2\right) \cdot / \mathrm{x} 1 \_22$;

Fminus $22=\exp (-\mathrm{K} 2 * \times \overline{2} 22) \cdot *(-$

$\left.1+2 * D 2 \bar{*}(z 02+2 * z b 2) *\left(\overline{1}+K 2 * x 2 \_22\right) \cdot / x 2 \_22 \cdot \wedge 2\right) \cdot / x 2 \_22 ;$

psio_22=1/(16*pi*D2).*(Fpluss_22+Fmiñus_22); \%cälculated reflectance for

secoñ known absorption at $2 \overline{0} \mathrm{~mm}$ distance

logpsi1_1=(log (psio_1)); $\frac{\circ}{\circ} \log$ constant for first known mua at $10 \mathrm{~mm}$ distance

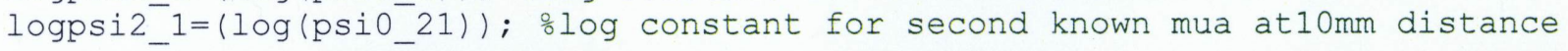

logpsi1_2=(log(psi0_12)); $\frac{\circ}{2} \log$ constant for first known mua at 20mm distance logpsi2_2=(log (psio_22)); $\frac{0}{\circ} \log$ constant for second known mua at $20 \mathrm{~mm}$ distance

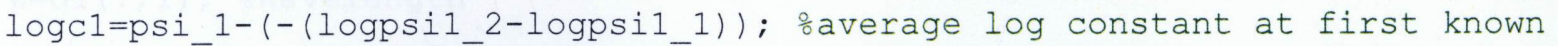
mua for both distances

logc2=psi_2-(-(logpsi2_2-logpsi2_1)); \%average log constant at second known mua for both distances

$\log _{C}=\left(\log _{C} 2+\log _{c} 1\right) / 2 ; \circ$ average $\log$ constant ratio

응 Calculate kappa

$\mathrm{i}=\mathrm{N}$;

PSI 1=PSI(i); omeasurement absorbance at specific wavelength

mus $\bar{p}=\operatorname{mus}(i) ; \quad$ specific scattering coefficient at specific wavelength 
$D=\left(1 . /\left(3 .{ }^{\star}\right.\right.$ musp $\left.)\right) ;$ odiffusion constant at specific wavelength

$\mathrm{zb}=2 .{ }^{*} \mathrm{D} ; \quad$ oboundary parameter

zO $=3 .{ }^{*}$; $\%$ boundary parameter

$\mathrm{x}_{1} 1=2$; $\frac{\circ}{8}$ larger $\mathrm{S} / \mathrm{D}$ distance

$\mathrm{x} \overline{1} 1=\operatorname{sqrt}\left(\mathrm{x}_{-} 1 \cdot \wedge^{\wedge} 2+\mathrm{z} 0 \cdot{ }^{\wedge} 2\right)$;

$x 21=\operatorname{sqrt}\left(x_{-}^{-1} \cdot \wedge 2+\left(z 0+2 \cdot{ }^{*} z b\right) \cdot \wedge 2\right) ;$

Eplus $=\exp \left(-K^{*} \times 11\right) \cdot *\left(1+2 * D * z 0 *\left(1+K^{*} \times 11\right) \cdot / x 11 \cdot \wedge 2\right) \cdot / x 11$;

Fminus $=\exp \left(-K^{*} \times 21\right) \cdot{ }^{*}\left(-1+2 * D^{*}(z 0+2 * z b) *\left(1+K^{*} \times 21\right) \cdot / \times 21 \cdot{ }^{\wedge} 2\right) \cdot / \times 21$;

psi_11=1/(16* pi*D).*(Fplus+Fminus); $\%$ calculated reflectance at $20 \mathrm{~mm}$ distance

$x \quad 2=1.0 ;$ ismaller S/D distance

$\mathrm{x} \overline{1} 2=\operatorname{sqrt}\left(\mathrm{x} 2 \mathbf{2}^{\wedge} 2+z 0 \cdot{ }^{\wedge} 2\right) ;$

$\mathrm{x} 22=\operatorname{sqrt}\left(\mathrm{x}^{-} 2 \cdot{ }^{\wedge} 2+\left(\mathrm{z} 0+2 \cdot{ }^{\star} \mathrm{zb}\right) \cdot{ }^{\wedge} 2\right) ;$

Fplus $2=\exp \left(-K^{\star} \times 12\right) \cdot{ }^{*}\left(1+2 * D^{\star} z 0 *(1+K * x 12) \cdot / x 12 \cdot{ }^{\wedge} 2\right) \cdot / x 12$;

Fminus $2=\exp \left(-K^{\star} \times 22\right) \cdot{ }^{*}\left(-1+2 * D^{*}(z 0+2 * z b) *(1+K * x 22) \cdot / \times 22 \cdot{ }^{\wedge} 2\right) \cdot / \times 22$;

distance

psi_22=1/(16*pi*D).*(Eplus2+Fminus 2$) ; \quad \% c a l c u l a t e d$ reflectance at $10 \mathrm{~mm}$

$\operatorname{logpsi}=\left(-\log \left(p s i \_11 . / p s i \_22\right)\right) ; \quad$ olog ratio to determine theoretical absorbance

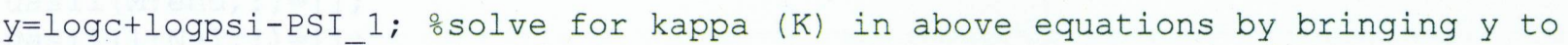
zero (fzero)

\section{2) Two-Layered Program}

o\% This program determines the optical properties of the top and bottom onlayer of a two-layered model by using linear regression fitting methods to o $\frac{\circ}{\circ}$ experimental data \%o.Written by Dr. Vladislav Toronov(May, 2008) o으일 by Olivia Pucci(September, 2008)

load db10_20 응 Absorbance data obtained from measurements

$\mathrm{d} 1=\mathrm{db} 10 \_20(350: 600,:)$;

$\mathrm{W}=\mathrm{d} 1(:, 1) ;$ oWavelength

$\frac{0}{0}$ ind optical properties of top layer

$[\mathrm{cb} 1, \operatorname{musp} 1]=\operatorname{fitTop}\left(\mathrm{w},\left(\mathrm{d} 1(:, 2) \cdot{ }^{\star} \log (10)\right),\left[\begin{array}{ll}10 & 20\end{array}\right]\right)$

\%Find optical properties of bottom layer

$[\mathrm{cb} 2$, musp2 $]=f i t B o t p h a n t o m\left(w,\left(d 1(:, 2) \cdot{ }^{*} \log (10)\right), 10,30, \mathrm{cb} 1\right.$, musp1 $)$

oThis code finds oxy- (ox1) and deoxy- (dx1) hemoglobin concentrations and reduced scattering coeff at $800 \mathrm{~nm}$ (musp1) assuming homogeneous tissue for the top layer 
function [cb1, mus1] =fitTop (lambda,psi, x)

load A oloads specific absorption data

load e; ocarbon black spectrum

alpha=1.3;

spe1=e;

$\frac{\circ}{5 p}(:, 1)=$ lambda; $\%$ Wavelength

osp $(:, 2)=($ interpl $(\operatorname{spe}(:, 1)$, spe $(:, 2)$, lambda $))$; $\%$ deoxy spectrum

osp $(:, 3)=($ interpl $(\operatorname{spe}(:, 1)$, spe $(:, 3)$, lambda $))$; \%oxy spectrum

osp $(:, 4)=($ interp1 ( $\operatorname{spe}(:, 1), \operatorname{spe}(:, 4)$, lambda $))$; $\%$ water spectrum

$\% \operatorname{sp}(:, 5)=($ interpl $(\operatorname{spe}(:, 1), \operatorname{spe}(:, 5)$, lambda $)) ; \%$ ICG spectrum

'This may take a few minutes'

load MATRIX2;

load uasill; $\%$ Silicone spectra

uasil=uasill.*0.1;

load intensity 20

load dark20

noise $=\operatorname{sqrt}($ intensity $20(:, 2)-\operatorname{dark} 20(:, 2))$; $\frac{\circ}{\circ}$ Noise

$\mathrm{N}=$ find ( $\operatorname{spe} 1(:, 1)>$ lambda (1), 1, 'first') ;

M=find ( $\operatorname{spe} 1(:, 1)>$ lambda (end), 1, 'first');

spel $(\mathrm{M}:$ end, $:)=[]$;

$\operatorname{spe} 1(1: \mathrm{N}-1,:)=[] ;$

uasil (M:end, : ) =[];

uasil $(1: \mathrm{N}-1,:)=[]$;

noise $(\mathrm{M}:$ end, $:)=[]$;

noise $(1: \mathrm{N}-1,:)=[]$;

$\mathrm{m} 1=\mathrm{find}(\operatorname{spe} 1(:, 1)==815)$;

$\mathrm{m} 2=\mathrm{find}(\operatorname{spe} 1(:, 1)==840)$;

$\mathrm{n} 1=\mathrm{find}(\operatorname{spe} 1(:, 1)==943)$;

$\mathrm{n} 2=$ find $(\operatorname{spe} 1(:, 1)==960)$;

Dpsi= (csaps ( $\operatorname{spe} 1(1:$ end-1,1), diff $(\operatorname{csaps}(\operatorname{spe} 1(:, 1)$, (interpl (lambda, smooth (psi), spe1(:,1))),0.05, spe1(:,1))),0.05, spe1(1:end-1,1))); \%Differential of the measured absorbance from experiments

\%Plot Measured Differential Absorbance Spectra

figure;

h=plot (spe1 (1:end-1,1), Dpsi, 'r') ;

$1=0$;

\% for $f t=0 .: 0.05: 0.3$;

$\mathrm{ft}=0$; $\div$ Fat fraction

for $i=1: 5$

'Initial localization'

$11=0$;

$\frac{\circ}{\circ}$ ind water fraction

for $w C 1=w C: .1$ : wC

$\mathrm{wC} 1=\mathrm{wC}$;

mua $=\operatorname{spe}(:, 2) * d x 1+\operatorname{spe}(:, 3) * o x 1+w c 1 * \operatorname{spe}(:, 4)+f t * \operatorname{spe}(:, 6)+i \operatorname{cg} 1 * \operatorname{spe}(:, 5)$;

mua $=w c 1 * \operatorname{spe}(:, 4)$;

Find scattering coefficient

for mus $1=0.1: 0.1: 1.1$ omsp800 cycle 
ㅇus $1=.07$;

muspl=musp $(\operatorname{mus} 1$, alpha, $\operatorname{spe} 1(:, 1))$;

for $\mathrm{cb}=150: 1: 350$ \%carbon black cycle

ofor ox=20:1:25 \%xyhemoglobin cycle

\%for $\mathrm{dx}=5: 1: 15$; odeoxyhemoglobin cycle

$$
\text { mua }=\operatorname{smooth}(\operatorname{spe} 1(:, 2), 5) * \mathrm{cb}+\operatorname{smooth}(\text { uasil, } 15) ; \frac{\circ}{\circ} \mathrm{Find}
$$

absorption coefficient for phantoms

omua $=\operatorname{spe}(:, 2) * d x+\operatorname{spe}(:, 3) * o x+w c 1 * \operatorname{spe}(:, 4)+f t * \operatorname{spe}(:, 6)$; $\%$ Find

absorption coefficient for heterogeneous tissue

ocompute spectrum of reflectance for given hemoglobin, water content and scattering

for $k=1$ : length (spe 1 )

$\operatorname{psi01}(k, 1)=\operatorname{PSI0}(\operatorname{mua}(k), \operatorname{musp} 1(k), x(1))$;

end

$\operatorname{psi02}(k, 1)=\operatorname{PSIO}(\operatorname{mua}(k), \operatorname{musp} 1(k), x(2))$;

$\mathrm{d}=0$;

DPSI $=\operatorname{diff}(\operatorname{smooth}((-\log ($ psi02./psi01) $), 10), 1)$;

stats $=$ regstats $\left(\left(\right.\right.$ Dpsi. ${ }^{\star}$ noise $(1$ : end -1$\left.)\right),\left(\right.$ DPSI.${ }^{*}$ noise $(1$ : end-

1)));

$$
\begin{array}{r}
d=\text { stats } \text {.rsquare; } \\
1=1+1 ; \\
s(1,:)=[d, c b, \text { mus } 1] ;
\end{array}
$$

end

end

$[\mathrm{ms}, \operatorname{imin}]=\min (s(:, 1))$;

Fitting parameters

$\mathrm{cb} 1=\mathrm{s}(\mathrm{imin}, 2)$; Carbon black

\% $\mathrm{Ox} 1=\mathrm{S}(\mathrm{imin}, 2) ; \% \mathrm{HbO} 2$

$\frac{\circ}{d x} 1=s(\operatorname{imin}, 3) ; \%$ HHb

mus1=s(imin, 3); oScattering coefficient

\%fat $=s($ imin, 5); $\%$ Fat

$\% \mathrm{wC}=\mathrm{s}($ imin, 6$) ;$ \% Water

$\mathrm{dd}=\mathrm{s}(\mathrm{imin}, \mathrm{:})$

clear $s$

$1=0$;

mua $=\operatorname{smooth}(\operatorname{spel}(:, 2), 5) * \operatorname{cb} 1+\operatorname{smooth}(u a s i l, 15)$;

omua $=\operatorname{spe}(:, 2) * d x 1+\operatorname{spe}(:, 3) * o x 1+w c^{*} \operatorname{spe}(:, 4)+$ fat*spe $(:, 6)$

musp1=musp (mus1, alpha, spe1 $(:, 1))$;

for $k=1$ : length (spe1)

$$
\operatorname{psi01}(k, 1)=\operatorname{PSIO}(\operatorname{mua}(k), \operatorname{musp} 1(k), x(1)) ;
$$


end

$$
\operatorname{psi02}(k, 1)=\operatorname{PSIO}(\operatorname{mua}(k), \operatorname{musp1}(k), \mathrm{x}(2)) \text {; }
$$

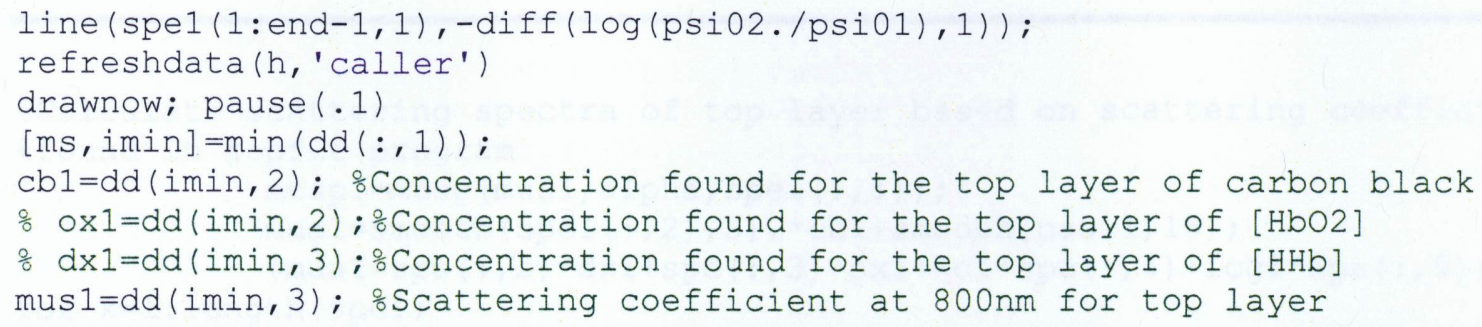

toc

end

oThis code finds oxy- (ox 1 ) and deoxy- (dx1) hemoglobin concentrations and reduced scattering coeff at $800 \mathrm{~nm}$ (muspl) assuming homogeneous otissue

function [cb2, mus2] =fitBotphantom (lambda, psi1, x1, x2, cb1, mus1)

\%function $[\mathrm{ox} 2, \mathrm{dx} 2$, mus2] = fitBot (lambda, psi1, psi2, x1, x2, ox1, dx1, mus1, wc1, wc2) oload A oloads specific absorption data

응 load Al;

oload cubic;

load e;

alpha=1.4;

oload op

spe1=e; \%cubic ;

load MATRIX2;

load uasil2; \%Silicon spectra

uasil=uasil2.*0.1;

load intensity 30

load dark30

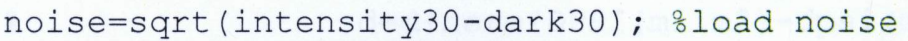

$\mathrm{N}=$ find ( $\operatorname{spe} 1(:, 1)>$ lambda (1), 1, 'first') ;

$M=f$ ind ( $\operatorname{spel}(:, 1)>$ lambda (end), 1, 'first') ;

spe $1(\mathrm{M}:$ end, $:)=[]$;

$\operatorname{spe} 1(1: \mathrm{N}-1,:)=[]$;

uasil $(\mathrm{M}:$ end, $:)=[]$;

uasil $(1: \mathrm{N}-1,:)=[]$;

noise $(M:$ end, $:)=[]$;

noise $(1: \mathrm{N}-1,:)=[]$;

ouasil=smooth (uasil, 15);

$\mathrm{m}=\mathrm{find}(\mathrm{x}=\mathrm{x} 1)$;

$\mathrm{n}=\mathrm{find}(\mathrm{x}=\mathrm{x} 2)$;

$\mathrm{ml}=\mathrm{find}(\operatorname{spe} 1(:, 1)==770)$;

$\mathrm{m} 2=\mathrm{find}(\operatorname{spe} 1(:, 1)==870)$;

DPSI $=(\langle$ csaps $(\operatorname{spe} 1(1:$ end -

$1,1)$, diff (csaps ( $\operatorname{spe} 1(:, 1)$, (interp1 (lambda, smooth (psi1, 15), spe1 (:,1))), 0.05, sp 


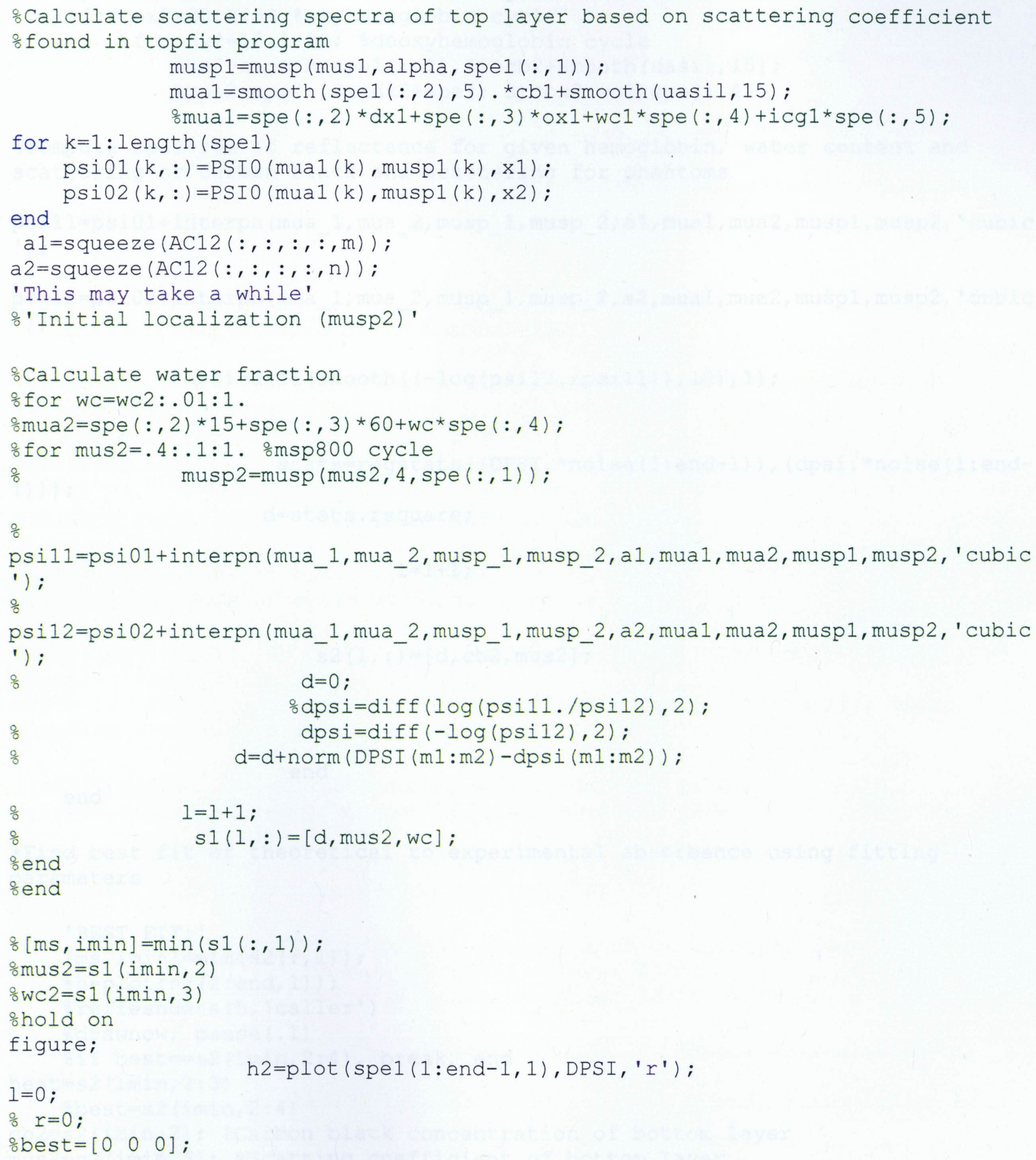


musp2=musp $($ mus2, alpha, $\operatorname{spe} 1(:, 1))$;

-Localizing ox2, $\mathrm{dx} 2$ and $\mathrm{cb} 2$

for $\mathrm{cb} 2=150: 1: 250$; Carbon black cycle

ofor ox $2=30: 2: 50$ oxyhemoglobin cycle

ofor dx2=10:1:20; \%deoxyhemoglobin cycle

mua2 $=\operatorname{smooth}(\operatorname{spe} 1(:, 2), 5) * \mathrm{cb} 2+\operatorname{smooth}($ uasil, 15$)$;

omua2 $=\operatorname{spe}(:, 2) * d \times 2+\operatorname{spe}(:, 3) * o \times 2+w c 2 * \operatorname{spe}(:, 4)$;

ocompute spectrum of reflectance for given hemoglobin, water content and scattering or carbon black and scattering for phantoms

psi11=psi01+interpn(mua_1,mua_2,musp_1,musp_2,a1,mua1,mua2,musp1, musp2, 'cubic ') ;

psi12=psi02+interpn (mua_1,mua_2,musp_1,musp_2,a2,mua1,mua2,musp1,musp2, 'cubic ') ;

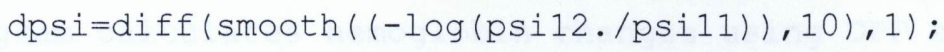

1))!;

stats $=$ regstats $\left(\left(\right.\right.$ DPSI.${ }^{*}$ noise $(1$ : end -1$\left.)\right),\left(\right.$ dpsi.${ }^{\star}$ noise $(1$ : end$d=$ stat. .rsquare;

$$
1=1+1 \text {; }
$$

$s 2(1,:)=[d, c b 2, \operatorname{mus} 2]$

end

end

sFind best fit of theoretical to experimental absorbance using fitting parameters

\section{'BEST FIT:'}

$[\mathrm{ms}, \operatorname{imin}]=\min (\mathrm{s} 2(:, 1))$;

o $\mathrm{h}=\mathrm{plot}(\mathrm{s} 2(2: \mathrm{end}, 1))$;

orefreshdata ( $h$, 'caller')

odrawnow; pause (.1)

\% if best $==$ s2 (imin, $2: 4)$, break, end best $=$ s $2(\operatorname{imin}, 2: 3)$

obest $=$ s2 $($ imin, $2: 4)$

$\mathrm{cb} 2=\mathrm{s} 2($ imin, 2$)$; $\frac{\circ}{\mathrm{c}}$ Carbon black concentration of bottom layer mus $2=s 2($ imin, 3$)$; oscatting coefficient of bottom layer

$\circ$ ox $2=\mathrm{s} 2(\mathrm{imin}, 2) ; \%[\mathrm{HbO} 2]$ of bottom layer

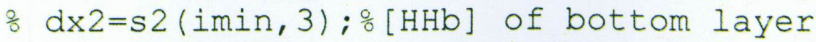

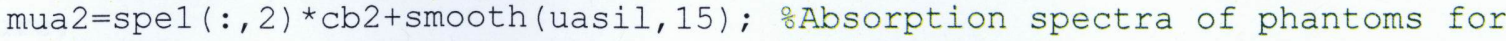
bottom layer 


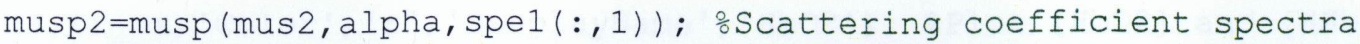

$\frac{\circ}{m} u a 2=\operatorname{spe}(:, 2) * d \times 2+\operatorname{spe}(:, 3) * o \times 2+w c 2 * \operatorname{spe}(:, 4) ;$ oAbsorption spectra of heterogeneous tissue for bottom layer

psi11=psi01+interpn(mua_1,mua_2,musp_1,musp_2,a1,mua1,mua2,musp1,musp2, 'cubic 1) ;

psi12=psi02+interpn (mua_1, mua_2, musp_1,musp_2,a2, mua1, mua2, musp1, musp2, 'cubic ') ;

h2=line $(\operatorname{spe} 1(1:$ end $-1,1)$, diff $(\operatorname{smooth}((-$

$\log (p s i 12 . / p s i 11)), 15), 1))$;

refreshdata(h2,'caller')Plot theoretical differential

absorbance

\section{3) Data Processing of Mobile Sensor Measurements}

응으은 This program calculates hemoglobin changes using DPF acquired from 음응wo-layered model and data acquired from mobile sensor

응 Written by:

으이ia Pucci and Dr. Vladislav Toronov (July 2009)

$\operatorname{dpf} 1=0.2227$; $\frac{\circ D F}{D}$ of brain at $730 \mathrm{~nm}$

$\mathrm{dpf} 2=0.1613$; $\frac{\mathrm{DPF}}{\text { of }} \mathrm{brain}$ at $830 \mathrm{~nm}$

a $730=-\log ([\operatorname{rest} 1(:, 3) \operatorname{rest1}(:, 6) \operatorname{rest1}(:, 9) \operatorname{rest} 1(:, 12)] . / \operatorname{dpf} 1) ;$ Data acquired from mobile sensor at $730 \mathrm{~nm}$

a $830=-\log ([\operatorname{rest} 1(:, 4) \operatorname{rest} 1(:, 7) \operatorname{rest1}(:, 10) \operatorname{rest} 1(:, 13)] . / \operatorname{dpf} 2) ;$ oData acquired from mobile sensor at $830 \mathrm{~nm}$

[trr730, deltar 730] =poldetrendM $(a 730,3) ;$ onetrend data

$\left[\operatorname{trr} 830\right.$, deltar_830] $=$ poldetrendM $(a 830,3)$; $\frac{\circ}{2}$ Detrend data

$\operatorname{EXD}=[3854.7,1879.8]$; oxtinction deoxy $[760,870]$

$\mathrm{EXO}=[1403.3,2875.2]$; $\frac{\circ}{\circ}$ extinction oxy $[760,870]$

denom $=\operatorname{EXO}(1) * \operatorname{EXD}(2)-\operatorname{EXO}(2) * \operatorname{EXD}(1) ; \%$ Denominator of Lamberts Beer Law deltar_ox $=1 e 6 *($ deltar_730*EXD(2)-deltar_830*EXD(1) ) / denom; deltar_dx $=-1 e 6 *($ deltar_830*EXO(1)-deltar_730*EXo(2) ) / denom;

$\operatorname{tr}$ ox $=(\operatorname{trr} 730 * \operatorname{EXD}(2)-\operatorname{trr} 830 * \operatorname{EXD}(1) \quad) \quad /$ denom; ocalculation of

[H̄̄o2] changes

$\operatorname{tr} d x=-(\operatorname{trr} 830 * \operatorname{EXO}(1)-\operatorname{trr} 730 * \operatorname{EXO}(2)$,$) / denom; oCalculation of$

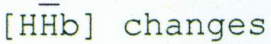

$t=\left(1:\right.$ length $\left.\left(t_{-} \_o x\right)\right) * .01 / 60 ; \%$ Time of measurements in seconds

figure； 


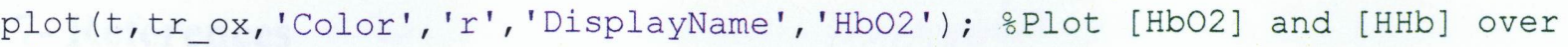
time

line $\left(t, t r \_d x\right)$; 


\section{7- References}

[1] Wang, L.V., \& H. Wu. Biomedical Optics: Principles and Imaging. New Jersey: John Wiley \& Sons, Inc. (2007).

[2] Jobsis, F.F. (1977). Noninvasive, infrared monitoring of cerebral and myocardial oxygen sufficiency and circulatory parameters. Science (New York, N.Y.) 198, 1264-1267.

[3] Aydin, E.D., C.R. de Oliveira \& A.J. Goddard. (2002). A comparison between transport and diffusion calculations using a finite element-spherical harmonics radiation transport method. Medical Physics 29, 2013-2023.

[4] Hecht, E. Optics, $4^{\text {th }}$ ed. San Francisco: Pearson Education Inc. (2002).

[5] Taroni, P., A. Pifferi, A. Torricelli, D. Comelli \& R. Cubeddu. (2003). In vivo absorption and scattering spectroscopy of biological tissues. Photochemical \& Photobiological Sciences : Official Journal of the European Photochemistry Association and the European Society for Photobiology 2, 124-129.

[6] Goode, T.L. \& H.J. Klein. (2002). Miniaturization: An overview of biotechmologiies for monitoring the physiology and pathophysiology of rodent animal models. ILAR Journal 43, 136-146.

[7] Fantini, S., M.A. Franceschini \& E. Gratton. (1994). Semi-infinite-geometry boundary problem for light migration in highly scattering media: a frequency-domain study in the diffusion approximation. Journal of the Optical Society of America 11, 2128-2138. 
[8] Kim, A.D. \& J.B. Keller. (2003). Light propagation in biological tissue. Journal of the Optical Society of America.A, Optics, Image Science, and Vision 20, 92-98.

[9] Alexandrakis, G., T.J. Farrell \& M.S. Patterson. (2000). Monte carlo diffusion hybrid model for photon migration in a two-layer turbid medium in the frequency domain. Applied Optics $39,2235-2244$.

[10] Gratton, E., S. Fantini, M.A. Franceschini, G. Gratton \& M. Fabiani. (1997). Measurements of scattering and absorption changes in muscle and brain. Philosophical Transactions of the Royal Society of London.Series B, Biological Sciences 352, 727-735.

[11] Groenhuis, R.A., H.A. Ferwerda \& J.J. Ten Bosch. (1983). Scattering and absorption of turbid materials determined from reflection measurements. 1: Theory. Applied Optics 22, 2456.

[12] Izzetoglu, K., S. Bunce, M. Izzetoglu, B. Onaral \& K. Pourrezaei. (2004). Functional nearinfrared neuroimaging. Conference Proceedings :...Annual International Conference of the IEEE Engineering in Medicine and Biology Society.IEEE Engineering in Medicine and Biology Society. Conference 7, 5333-5336.

[13] Piantadosi, C.A. (2007). Early development of near-infrared spectroscopy at Duke University. Journal of Biomedical Optics 12, 062102.

[14] Benni, P. B., et al. (1995). A novel near-infrared spectroscopy (NIRS) system for measuring regional oxygen saturation. IEEE 21st Annual Northeast, 105 - 107. 
[15] Bozkurt, A., A. Rosen, H. Rosen \& B. Onaral. (2005). A portable near infrared spectroscopy system for bedside monitoring of newborn brain. Biomedical Engineering Online 4, 29.

[16] Bunce, S.C., M. Izzetoglu, K. Izzetoglu, B. Onaral \& K. Pourrezaei. (2006). Functional near-infrared spectroscopy. IEEE Engineering in Medicine and Biology Magazine : The Quarterly Magazine of the Engineering in Medicine \& Biology Society 25, 54-62.

[17] Jacob, M., Y. Bresler, V. Toronov, X. Zhang \& A. Webb. (2006). Level-set algorithm for the reconstruction of functional activation in near-infrared spectroscopic imaging. Journal of Biomedical Optics 11, 064029.

[18] Villringer, A. \& B. Chance. (1997). Non-invasive optical spectroscopy and imaging of human brain function. Trends in Neurosciences 20, 435-442.

[19] Rolfe, P. (2000). In vivo near-infrared spectroscopy. Annual Review of Biomedical Engineering 2, 715-754.

[20] Chance, B., E. Anday, S. Nioka, S. Zhou, L. Hong, K. Worden, C. Li, et al. (1998). A novel method for fast imaging of brain function, non-invasively, with light. Optics Express 2, 411423.

[21] Bozkurt, A. \& B. Onaral. (2004). Safety assessment of near infrared light emitting diodes for diffuse optical measurements. Biomedical Engineering Online 3, 9. 
[22] Workman, J. \& L. Weyer. Practical Guide to Interpretive Near-Infrared Spectroscopy. Florida: Taylor \& Francis Group (2008).

[23] Brown, K.R., B.M. Brown, E. Hoagland, C.L. Mayne \& E.L. Hegg. (2004). Heme A synthase does not incorporate molecular oxygen into the formyl group of heme A. Biochemistry 43, 8616-8624.

[24] Caughey, W.S., G.A. Smythe, D.H. O'Keeffe, J.E. Maskasky \& M.I. Smith. (1975). Heme A of cytochrome c oxicase. Structure and properties: comparisons with hemes B, C, and S and derivatives. The Journal of Biological Chemistry 250, 7602-7622.

[25] Mader, S.S. Biology $8^{\text {th }}$ Ed. New York: McGraw-Hill Science/Engineering/Math. (2003).

[26] Schmitt, J.M. Optical Measurement of Blood Oxygenation by Implantable Telemetry. Technical Report G558-15, Stanford.

[27] Moaveni, M.K. "A multiple scattering field theory applied to whole blood". (Ph.D. dissertation, University of Washington, 1970).

[28] R.L.P. Van Veen, R.L.P, H.J.C.M. Sterenborg, A. Pifferi, A. Torricelli \& R. Cubeddu. Determination of VIS- NIR absorption coefficients of mammalian fat, with time and spatially resolved diffuse reflectance and transmission spectroscopy. OSA Annual BIOMED Topical Meeting, 2004.

[29] Palmer, K.F. \& D. Williams. (1974). Optical properties of water in the near infrared. Journal of the Optical Society of America 64, 1107-1110. 
[30] Izzetoglu, M., S.C. Bunce, K. Izzetoglu, B. Onaral \& K. Pourrezaei. (2007). Functional brain imaging using near-infrared technology. IEEE Engineering in Medicine and Biology Magazine: The Quarterly Magazine of the Engineering in Medicine \& Biology Society 26, $38-46$.

[31] Boas, D.A., T. Gaudette, G. Strangman, X. Cheng, J.J. Marota \& J.B. Mandeville. (2001). The accuracy of near infrared spectroscopy and imaging during focal changes in cerebral hemodynamics. NeuroImage 13, 76-90.

[32] Kim, J.G., M. Xia \& H. Liu. (2005). Extinction coefficients of hemoglobin for nearinfrared spectroscopy of tissue. IEEE Engineering in Medicine and Biology Magazine : The Quarterly Magazine of the Engineering in Medicine \& Biology Society 24, 118-121.

[33] Germon, T.J., Young, A.E., Nelson, R.J. (1995). Near-infrared spectroscopy. Journal of Neurosurgery 83(6), 1111-12.

[34] Muehlemann, T., D. Haensse \& M. Wolf. (2008). Wireless miniaturized in-vivo near infrared imaging. Optics Express 16, 10323-10330.

[35] Hull, E.L., M.G. Nichols \& T.H. Foster. (1998). Quantitative broadband near-infrared spectroscopy of tissue-simulating phantoms containing erythrocytes. Physics in Medicine and Biology 43, 3381-3404.

[36] Li, A., R. Kwong, A. Cerussi, S. Merritt, C. Hayakawa \& B. Tromberg. (2007). Method for recovering quantitative broadband diffuse optical spectra from layered media. Applied Optics 46, 4828-4833. 
[37] Steinhoff B.J., G. Herrendorf \& C. Kurth. (1996). Ictal near infrared spectroscopy in temporal lobe epilepsy: a pilot study. Seizure 5, 97-101.

[38] Kirkpatrick P.J. (1997). Use of near-infrared spectroscopy in the adult. Philos. Trans. R. Soc. Lond. B Biol. Sci. 352, 701-705.

[39] Germon, T.J., P.D. Evans, A.R. Manara, N.J. Barnett, P. Wall \& R.J. Nelson. (1998). Sensitivity of near infrared spectroscopy to cerebral and extra-cerebral oxygenation changes is determined by emitter-detector separation. Journal of Clinical Monitoring and Computing 14, $353-360$.

[40] Chance, B., P. Cohen, F. Jobsis \& B. Schoener. (1962). Intracellular oxidation-reduction states in vivo. Science $137,499-508$.

[41] Kramer, K. (1935). Verfahren zur fortlaufenden messung des sauerstoffgehaltes im stromenden blute an uneroffneten gefassen. Z. Biol. 96,61-75.

[42] Brazy, J.E., D.V. Lewis, M.H. Mitnick \& F.F. Jobsis vander Vliet. (1985). Noninvasive monitoring of cerebral oxygenation in preterm infants: preliminary observations. Pediatrics $75,217-225$.

[43] Young, A.E., T.J. Germon, N.J. Barnett, A.R. Manara \& R.J. Nelson. (2000). Behaviour of near-infrared light in the adult human head: implications for clinical near-infrared spectroscopy. British Journal of Anaesthesia 84, 38-42. 
[44] Gratton, E., V. Toronov, U. Wolf, M. Wolf \& A. Webb. (2005). Measurement of brain activity by near-infrared light. Journal of Biomedical Optics 10, 11008.

[45] Wolf, M. et. al. (2008). Near infrared spectroscopy to study the brain: an overview. Opto-Electronics Review 16, 413-419.

[46] Stepnoski, R.A., A. LaPorta, F. Raccuia-Behling, G.E. Blonder, R.E. Slusher \& D. Kleinfeld. (1991). Noninvasive detection of changes in membrane potential in cultured neurons by light scattering. Proceedings of the National Academy of Sciences of the United States of America 88, 9382-9386.

[47] Strangman, G., D.A. Boas \& J.P. Sutton. (2002). Non-invasive neuroimaging using nearinfrared light. Biological Psychiatry 52, 679-693.

[48] Franceschini, M.A. \& D.A. Boas. (2004). Noninvasive measurement of neuronal activity with near-infrared optical imaging. NeuroImage 21, 372-386.

[49] Hamalainen, M.S. (1992). Magnetoencephalography: a tool for functional brain imaging. Brain Topography 5, 95-102.

[50] Ter-Pogossian, M.M., M. E. Phelps, \& E.J. Hoffman. (1975). A positron-emission transaxial tomograph for nuclear imaging (PET). Radiology 114, 89-98.

[51] Phelps, M.E., E.J. Hoffman, N.A. Mullani, \& M.M. Ter-Pogossian. (1975). Application of annihilation coincidence detection to transaxial reconstruction tomography. Journal of Nuclear Medicine 16, 210-224. 
[52] Positron Emission Tomography. Found at http://legacyweb.triumf.ca/welcome/petscan. html on July 27, 2009.

[53] Ibaraki, M., S. Miura, E. Shimosegawa, S. Sugawara, T. Mizuta, A. Ishikawa \& M. Amano. (2008). Quantification of cerebral blood flow and oxygen metabolism with 3-dimensional PET and 15O: validation by comparison with 2-dimensional PET. Journal of Nuclear Medicine : Official Publication, Society of Nuclear Medicine 49, 50-59.

[54] Davidson, R.J. \& S.K. Sutton. (1995). Affective neuroscience: the emergence of a discipline. Current Opinion in Neurobiology 5, 217-224.

[55] Bennett, L. H., Page, C. H. \& L.J. Swartzendruber (1978). Comments on units in magnetism. Journal of Research of the National Bureau of Standards 83, 9-12.

[56] Heremans, J., C. H. Olk, \& D. T. Morelli (1994). Magnetic Susceptibility of Carbon Structures. Phys. Rev. B 49, 15122-15125.

[57] Toronov, V., S. Walker, R. Gupta, J.H. Choi, E. Gratton, D. Hueber \& A. Webb. (2003). The roles of changes in deoxyhemoglobin concentration and regional cerebral blood volume in the fMRI BOLD signal. NeuroImage 19, 1521-1531.

[58] Wolf, M., U. Wolf, V. Toronov, A. Michalos, L.A. Paunescu, J.H. Choi \& E. Gratton. (2002). Different time evolution of oxyhemoglobin and deoxyhemoglobin concentration changes in the visual and motor cortices during functional stimulation: a near-infrared spectroscopy study. NeuroImage 16, 704-712. 
[59] Schünke, M., M. Schünke, E. Schulte, U. Schumacher, J. Rude, L.M. Ross, E.D. Lamperti, et al. Head and neuroanatomy. Anonymous In "Thieme atlas of anatomy.". Stuttgart ; New York:Thieme (2007).

[60] Kienle, A., M.S. Patterson, N. Dognitz, R. Bays, G. Wagninures \& H. van den Bergh. (1998). Noninvasive Determination of the Optical Properties of Two-Layered Turbid Media. Applied Optics 37, 779-791.

[61] Alexandrakis, G., T.J. Farrell \& M.S. Patterson. (1998). Accuracy of the diffusion approximation in determining the optical properties of a two-layer turbid medium. Applied Optics 37, 7401-7409.

[62] Li, A., R. Kwong, A. Cerussi, S. Merritt, C. Hayakawa \& B. Tromberg. (2007). Method for recovering quantitative broadband diffuse optical spectra from layered media. Applied Optics 46, 4828-4833.

[63] Chance, B., Z. Zhuang, C. UnAh, C. Alter \& L. Lipton. (1993). Cognition-activated lowfrequency modulation of light absorption in human brain. Proceedings of the National Academy of Sciences of the United States of America 90,3770-3774.

[64] Fukui, Y., Y. Ajichi \& E. Okada. (2003). Monte Carlo prediction of near-infrared light propagation in realistic adult and neonatal head models. Applied Optics 42, 2881-2887.

[65] Okada, E. \& D.T. Delpy. (2003). Near-infrared light propagation in an adult head model. I. Modeling of low-level scattering in the cerebrospinal fluid layer. Applied Optics 42, 29062914. 
[66] Zhang, X. "Simultaneous integrated functional magnetic resonance imaging and optical tomography in the near infrared spectrum (sinfonis) for studying hemodynamic and neuronal responses in the human brain". (Ph.D. dissertation, University of Illinois, 2005), p. 104.

[67] Sterenborg,H., M. van Gemert, W. Kamphorst, J. Wolbers, and W. Hogervorst. (1989). The spectral dependence of the optical properties of human brain. Lasers in Medical Science 4, 221-227.

[68] Okada, E., M. Firbank, M. Schweiger, S. Arridge, M. Cope, and D. Delpy. (1997). Theoretical and experimental investigation of near-infrared light propagation in a model of the adult head. Applied Optics 36, 21-31.

[69] W. Gottschalk, W. “ Ein messverfahren zur bestimmung der optischen parameter biologischer gevebe in vitro". (Ph.D. dissertation, Universitaet Fridriciana, Karlsruhe, 1993).

[70] Roggan, A., O. Minet, C. Schroeder, \& G. Mueller. (1994). Determination of optical tissue properties with double integrating sphere technique and Monte Carlo simulations. Proceedings in SPIE 2100, 42-56.

[71] Yaroslavsky, A.N., P. C. Schulze, I. V. Yaroslavsky, R. Schober, F. Ulrich, and H.-J. Schwarzmaier. (2002). Optical properties of selected native and coagulated human brain tissue in vitro in the visible and near-infrared spectral range. Physics in Medicine and Biology 47, 2059-2073. 
[72] Strangman, G., M. Franceschini, and D. Boas. (2003). Factors affecting the accuracy of near-infrared spectroscopy concentration calculations for focal changes in oxygenation parameters. NeuroImage 18, 865-879.

[73] Choi, J., M. Wolf, V. Toronov, U. Wolf, C. Polzonetti, D. Hueber, L. Safonova, R. Gupta, A. Michalos, W. Mantulin, and E. Gratton. (2004). Noninvasive determination of the optical properties of adult brain: Near-infrared spectroscopy approach. Journal of Biomedical Optics 9, 221-229.

[74] Bevilacqua, F., D. Piguet, P. Marquet, J. Gross, B. Tromberg, and C. Depeursinge. (1999). In vivo local determination of tissue optical properties: Applications to human brain. Applied Optics 38, 4939-4950.

[75] Splinter, R., W. Cheong, M. van Gemert, and A. Welch. (1989). In vivo optical properties of human and canine brain and urinary bladder tissues at $633 \mathrm{~nm}$. Lasers in Surgery and Medicine 9, 37-41.

[76] Carmines, E.L. (2002). Evaluation of the potential effects of ingredients added to cigarettes. Part 1: Cigarette design, testing approach, and review of results. Food and Chemical Toxicology 40(1), 77-91.

[77] Heath Canada (2007) Canadian Tobacco Use Monitoring Survey, Health Canada, Ottawa.

[78] Giessing, C., et al. (2006). The modulatory effects of nicotine on parietal cortex activity in a cued target detection task depend on cue reliability. Neuroscience 137(3), 853-64. 
[79] Siafaka, A., et al. (2007). Acute effects of smoking on skeletal muscle microcirculation monitored by near-infrared spectroscopy. Chest Journal 131(5), 1479-85.

[80] Frederick, B., et al. (2007). An MR-compatible device for delivering smoked marijuana during functional imaging. Pharmacology, Biochemistry, and Behavior 87(1), 81-89.

[81] Birkner, T. et. al. (2002). Acute effects of cigarette smoking on cerebral oxygenation and hemodynamics: A combined study with near-infrared spectroscopy and transcranial Doppler sonography. Journal of Neurological Sciences 205, 71-75.

[82] Brody, A.L., et al. (2006). Cigarette smoking saturates brain alpha4beta2 nicotinic acetylcholine receptors. Archives of General Psychiatry 63, 907-915.

[83] Sharieh S., Ferworn, A., and Toronov, V. (2008). A GSM mobile system to monitor brain function using a near-infrared light sensor, 000665 -000668, Canadian Conference on Electrical and Computer Engineering, IEEE, 2008.

[84] Zhao, H. et. al. (2002). Maps of optical differential pathlength factor of human adult forehead, somatosensory motor and occipital regions at multi-wavelengths in NIR. Physics in Medicine and Biology 47, 2075-2093.

[85] Toronov, V., et al. (2001). Investigation of human brain hemodynamics by simultaneous near-infrared spectroscopy and functional magnetic resonance imaging. Medical Physics 28(4), 521-27. 
[86] Alexandrakis, G., D.R. Busch, G.W. Faris \& M.S. Patterson. (2001). Determination of the optical properties of two-layer turbid media by use of a frequency-domain hybrid Monte Carlo diffusion model. Applied Optics 40, 3810-3821.

[87] Kienle, A., M.S. Patterson, N. Dognitz, R. Bays, G. Wagninures \& H. van den Bergh. (1998). Noninvasive Determination of the Optical Properties of Two-Layered Turbid Media. Applied Optics 37, 779-791.

[88] Hoffmann, D., Hoffmann, I., El-Bayoumy, K. (2001) The less harmful cigarette: A controversial issue. A tribute to Ernst L. Wynder. Chemical Research in Toxicology 14(7), 767-90.

[89] Baker, R.R., Massey, E.D., Smith, G. (2004). An overview of the effects of tobacco ingredients on smoke chemistry and toxicity. Food and Chemical Toxicology 42S, S53-S83.

[90] Rodgman, A., Smith, C.J., Perfetti, T.A. (2000). The composition of cigarette smoke: a retrospective, with emphasis on polycyclic components. Human and Experimental Toxicology 19(10), 573-95.

[91] Billet, C. \& R. Sablong. (2007). Differential optical spectroscopy for absorption characterization of mono \& two-layered scattering media. Conference Proceedings : ...Annual International Conference of the IEEE Engineering in Medicine and Biology Society.IEEE Engineering in Medicine and Biology Society. Conference 2007, 2622-2625.

[92] Cabeza, R. \& L. Nyberg. (2000). Imaging cognition II: An empirical review of 275 PET and fMRI studies. Journal of Cognitive Neuroscience 12, 1-47. 
[93] De Blasi, R.A., M. Ferrari, A. Natali, G. Conti, A. Mega \& A. Gasparetto. (1994).

Noninvasive measurement of forearm blood flow and oxygen consumption by near-infrared spectroscopy. Journal of Applied Physiology (Bethesda, Md.: 1985) 76, 1388-1393.

[94] Matcher, S.J., M. Cope \& D.T. Delpy. (1994). Use of the water absorption spectrum to quantify tissue chromophore concentration changes in near-infrared spectroscopy. Physics in Medicine and Biology 39, 177-196.

[95] Okada, E. \& D.T. Delpy. (2003). Near-infrared light propagation in an adult head model. II. Effect of superficial tissue thickness on the sensitivity of the near-infrared spectroscopy signal. Applied Optics 42, 2915-2922.

[96] Pucci, O, S. Sharieh \& V. Toronov. (2008). Spectral and spatial characteristics of the differential pathlengths in non-homogeneous tissues. Proceedings of SPIE 6855,685506-1-7.

[97] Thiagarajah, J. R., Papadopoulos, M. C., Verkman, A.S. (2005). Noninvasive early detection of brain edema in mice by near-infrared light scattering. Journal of neuroscience research 80(2), 293-299.

[98] Toronov, V. et al. (2000). Near-infrared study of fluctuations in cerebral hemodynamics during rest and motor stimulation: temporal analysis and spatial mapping. Medical physics 27(4), 801-815.

[99] Xu, H., R. Springett, H. Dehghani, B.W. Pogue, K.D. Paulsen \& J.F. Dunn. (2005). Magnetic-resonance-imaging-coupled broadband near-infrared tomography system for small animal brain studies. Applied Optics 44, 2177-2188. 
[100] Yoshitani, K., M. Kawaguchi, N. Miura, T. Okuno, T. Kanoda, Y. Ohnishi \& M. Kuro. (2007). Effects of hemoglobin concentration, skull thickness, and the area of the cerebrospinal fluid layer on near-infrared spectroscopy measurements. Anesthesiology 106, $458-462$.

[101] Xu, H, et al. (2003). Feasibility of NIR tomographic reconstruction with multispectral continuous wave data by mapping into frequency domain data. Proceedings of SPIE 4955, $103-114$. 